ANALYSIS \& PDE Volume $8 \quad$ No. $6 \quad 2015$ LUC MOLINET AND STÉPHANE VENTO

IMPROVEMENT OF THE ENERGY METHOD FOR STRONGLY NONRESONANT DISPERSIVE EQUATIONS AND APPLICATIONS 


\title{
IMPROVEMENT OF THE ENERGY METHOD FOR STRONGLY NONRESONANT DISPERSIVE EQUATIONS AND APPLICATIONS
}

\author{
Luc Molinet and Stéphane Vento
}

\begin{abstract}
We propose a new approach to prove the local well-posedness of the Cauchy problem associated with strongly nonresonant dispersive equations. As an example, we obtain unconditional well-posedness of the Cauchy problem in the energy space for a large class of one-dimensional dispersive equations with a dispersion that is greater than the one of the Benjamin-Ono equation. At the level of dispersion of the Benjamin-Ono, we also prove the well-posedness in the energy space but without unconditional uniqueness. Since we do not use a gauge transform, this enables us in all cases to prove strong convergence results in the energy space for solutions of viscous versions of these equations towards the purely dispersive solutions. Finally, it is worth noting that our method of proof works on the torus as well as on the real line.
\end{abstract}

\section{Introduction}

The Cauchy problem associated with dispersive equations with derivative nonlinearity has been extensively studied since the eighties. The first results were obtained by using energy methods that did not make use of the dispersive effects (see for instance [Kato 1983; Abdelouhab et al. 1989]). These methods were restricted to regular initial data ( $s>d / 2$, where $d \geq 1$ is the spatial dimension) and only ensured the continuity of the solution map. At the end of the eighties, Kenig, Ponce and Vega proved new dispersive estimates that enable them to lower the regularity requirement on the initial data (see for instance [Kenig et al. 1991; 1993; Ponce 1991]). They even obtained local well-posedness (LWP) for a large class of dispersive equations by a fixed point argument in a suitable Banach space related to linear dispersive estimates. Then, Bourgain [1993a; 1993b] introduced the now so-called Bourgain spaces, where one can solve by a fixed point argument a wide class of dispersive equations with very rough initial data. It is worth noting that, since the nonlinearity of these equations is in general algebraic, the fixed point argument ensures the real analyticity of the solution map. Molinet, Saut and Tzvetkov [Molinet et al. 2001] noticed that a large class of "weakly" dispersive equations, including in particular the Benjamin-Ono equation, cannot be solved by a fixed point argument for initial data in any Sobolev spaces $H^{s}$. This obstruction is due to bad interactions between high frequencies and very low frequencies. Since then, roughly speaking, two approaches have been developed to lower the regularity requirement for such equations. The first one is the so-called gauge method. This consists in introducing a nonlinear gauge transform of the solution that solved an equation with fewer bad interactions than the original one. This method proved to be very

MSC2010: 35E15, 35Q53, 35A02.

Keywords: Benjamin-Ono equation, intermediate long wave equation, dispersion generalized Benjamin-Ono equation, well-posedness, unconditional uniqueness. 
efficient for obtaining the lowest regularity index for solving canonical equations (see [Tao 2004; Ionescu and Kenig 2007; Burq and Planchon 2008; Molinet and Pilod 2012] for the BO equation and [Herr et al. 2010] for the dispersive generalized BO equation) but has the disadvantage of behaving very badly with respect to perturbation of the equation. The second one consists in improving the dispersive estimates by localizing it in space-frequency-depending time intervals and then mixing it with classical energy estimates. This type of method was first introduced by Koch and Tzvetkov [2003] (see also [Kenig and Koenig 2003] for some improvements) in the framework of Strichartz's spaces and then by Koch and Tataru [2007] (see also [Ionescu et al. 2008]) in the framework of Bourgain's spaces. It is less efficient for getting the best regularity index but it is surely more flexible with respect to perturbation of the equation.

In this paper we propose a new approach to derive local and global well-posedness results for dispersive equations that do not exhibit too-strong resonances. This approach combines classical energy estimates with Bourgain-type estimates on a time interval that does not depend on the space frequency. Here, we will apply this method to prove unconditional local well-posedness results on both $\mathbb{R}$ and $\mathbb{T}=\mathbb{R} / 2 \pi \mathbb{Z}$ without the use of a gauge transform for a large class of one-dimensional quadratic dispersive equations with a dispersion between those of the Benjamin-Ono equation and the KdV equation. This class contains, in particular, the equations with pure power dispersion that read

$$
u_{t}+\partial_{x} D_{x}^{\alpha} u+u u_{x}=0
$$

with $\alpha \in[1,2]$.

The principle of the method is particularly simple in the regular case $s>\frac{1}{2}$. We start with the classical space-frequency-localized energy estimate

$$
\left\|P_{N} u\right\|_{L_{T}^{\infty} H^{s}}^{2} \lesssim\left\|P_{N} u_{0}\right\|_{H^{s}}^{2}+\sup _{t \in] 0, T[}\langle N\rangle^{2 s}\left|\int_{0}^{t} \int_{\mathbb{R}} \partial_{x} P_{N}\left(u^{2}\right) P_{N} u\right|,
$$

obtained by projecting the equation on frequencies of order $N$ and taking the inner product with $J_{x}^{s} u$. Note that the second term in the right-hand side of (1-2) is easily controlled (after summing in $N$ ) by $\|u\|_{L_{T}^{\infty} H^{s}}^{3}$ for $s>\frac{3}{2}$. This is the main point in the standard energy method that leads to LWP in $H^{s}, s>\frac{3}{2}$. In order to take into account the dispersive effects of the equation, we will decompose the three factors in the integral term into dyadic pieces for the modulation variables and use the Bourgain spaces $X^{s, b}$ in a nonconventional way. Actually, it is known that standard bilinear estimates in $X^{s, b}$-spaces with $b=\frac{1}{2}+$ fail for (1-1) for any $s \in \mathbb{R}$ as soon as $\alpha<2$. On the other hand, as noticed in [Zhou 1997], it is easy to deduce from the equation that a solution $u \in L^{\infty}\left(0, T ; H^{s}\right)$ to (1-3) has to belong to the space $X_{T}^{s-1,1}$. This means that, if we accept the loss of a few spatial derivatives on the solution, then we may gain some regularity in the modulation variable. This is particularly profitable when the equation enjoys a strong nonresonance relation such as (2-6). Actually, this formally allows us to estimate the second term in (1-2) at the desired level. However, this term involves a multiplication by $1_{0, t}[$ and it is well known that such multiplication is not bounded in $X^{s-1,1}$. To overcome this difficulty we decompose this function into two parts: a high-frequency part that will be very small in $L_{T}^{1}$ and a low-frequency part that will have good properties with respect to multiplication with high-modulation functions in $X^{s-1,1}$. This decomposition will depend on the space-frequency-localization of the three functions that appear in the trilinear term. 
1A. Presentation of the results. In this paper we consider the dispersive equation

$$
u_{t}+L_{\alpha+1} u+\frac{1}{2} \partial_{x}\left(u^{2}\right)=0
$$

associated with the initial condition

$$
u(0, \cdot)=u_{0},
$$

where $x \in \mathbb{R}$ or $\mathbb{T}, u=u(t, x)$ and $u_{0}=u_{0}(x)$ are real-valued functions, $\alpha>0$ is a real number and the linear operator $L_{\alpha+1}$ satisfies the following hypothesis:

Hypothesis 1. $L_{\alpha+1}$ is the Fourier multiplier operator by i $p_{\alpha+1}$, where $p_{\alpha+1}$ is a real-valued odd function satisfying, for some $\lambda_{0}>0$,

(1) For any $|\xi| \gg 1$ and $0<\lambda \leq \lambda_{0}$,

$$
\lambda^{\alpha+1}\left|p_{\alpha+1}\left(\lambda^{-1} \xi\right)\right| \lesssim|\xi|^{\alpha+1}
$$

(2) For any $\left(\xi_{1}, \xi_{2}\right) \in \mathbb{R}^{2}$ with $\left|\xi_{1}\right| \gg 1$ and any $0<\lambda \leq \lambda_{0}$,

$$
\lambda^{\alpha+1}\left|\Omega\left(\lambda^{-1} \xi_{1}, \lambda^{-1} \xi_{2}\right)\right| \sim|\xi|_{\min }|\xi|_{\max }^{\alpha},
$$

where

$$
\begin{aligned}
\Omega\left(\xi_{1}, \xi_{2}\right) & :=p_{\alpha+1}\left(\xi_{1}+\xi_{2}\right)-p_{\alpha+1}\left(\xi_{1}\right)-p_{\alpha+1}\left(\xi_{2}\right), \\
|\xi|_{\min } & :=\min \left(\left|\xi_{1}\right|,\left|\xi_{2}\right|,\left|\xi_{1}+\xi_{2}\right|\right) \\
\text { and } \mid \xi_{\max } & :=\max \left(\left|\xi_{1}\right|,\left|\xi_{2}\right|,\left|\xi_{1}+\xi_{2}\right|\right) .
\end{aligned}
$$

Remark 1.1. We will see in Lemma 2.1 below that, for $\alpha>0$, a very simple criterion on $p$ ensures (1-6). With this criterion in hand, it is not too hard to check that the following linear operators satisfy Hypothesis 1 :

(1) The purely dispersive operators $L:=\partial_{x} D_{x}^{\alpha}$ with $\alpha>0$.

(2) The linear intermediate long wave operator $L:=\partial_{x} D_{x} \operatorname{coth} D_{x}$. Note that here $\alpha=1$.

(3) Some perturbations of the Benjamin-Ono equation, such as the Smith operator [1972], $L:=$ $\partial_{x}\left(D_{x}^{2}+1\right)^{1 / 2}$. Here again $\alpha=1$.

Before stating our main result, let us define what we mean by unconditional well-posedness.

Definition 1.2. Let $\mathbb{K}=\mathbb{R}$ or $\mathbb{T}, T>0$ and $s \geq 0$. We will say that $u \in L^{\infty}\left(0, T ; H^{s}(\mathbb{K})\right)$ is a solution to (1-3) associated with the initial datum $u_{0} \in H^{s}(\mathbb{K})$ if $u$ satisfies (1-3)-(1-4) in the distributional sense, i.e., for any test function $\phi \in C_{c}^{\infty}(]-T, T[\times \mathbb{K})$,

$$
\int_{0}^{\infty} \int_{\mathbb{K}}\left[\left(\phi_{t}+L_{\alpha+1} \phi\right) u+\frac{1}{2} \phi_{x} u^{2}\right] d x d t+\int_{\mathbb{K}} \phi(0, \cdot) u_{0} d x=0
$$

Remark 1.3. For $u \in L^{\infty}\left(0, T ; H^{s}(\mathbb{K})\right)$, with $s \geq 0, u^{2}$ is well defined and is in $L^{\infty}\left(0, T ; H^{s-(1 / 2+)}(\mathbb{K})\right)$. Moreover, (1-5) forces

$$
L_{\alpha+1} u \in L^{\infty}\left(0, T ; H^{s-\alpha-1}(\mathbb{K})\right) .
$$

Therefore, $u_{t} \in L^{\infty}\left(0, T ; H^{s-\alpha-1}(\mathbb{K})\right)$ and (1-7) ensures that (1-3) is satisfied in $L^{\infty}\left(0, T ; H^{s-\alpha-1}(\mathbb{K})\right)$. In particular, $u \in C\left([0, T] ; H^{s-\alpha-1}(\mathbb{K})\right)$ and (1-7) forces the initial condition $u(0)=u_{0}$. Note that this 
actually implies that $u \in C\left([0, T] ; H^{\theta}(\mathbb{K})\right)$ for any $\theta<s$. Finally, we note that this ensures that $u$ satisfies the Duhamel formula associated with (1-3).

Definition 1.4. Let $\mathbb{K}=\mathbb{R}$ or $\mathbb{T}$ and $s \in \mathbb{R}$. We will say that the Cauchy problem associated with (1-3) is unconditionally locally well-posed in $H^{s}(\mathbb{K})$ if, for any initial data $u_{0} \in H^{s}(\mathbb{K})$, there exists $T=$ $T\left(\left\|u_{0}\right\|_{H^{s}}\right)>0$ and a solution $u \in C\left([0, T] ; H^{s}(\mathbb{K})\right)$ to (1-3) emanating from $u_{0}$. Moreover, $u$ is the unique solution to (1-3) associated with $u_{0}$ that belongs to $L^{\infty}(] 0, T\left[; H^{s}(\mathbb{K})\right)$. Finally, for any $R>0$, the solution map $u_{0} \mapsto u$ is continuous from the ball of $H^{s}(\mathbb{K})$ with radius $R$ centered at the origin into $C\left([0, T(R)] ; H^{s}(\mathbb{K})\right)$.

Theorem 1.5. Let $\mathbb{K}=\mathbb{R}$ or $\mathbb{T}, L_{\alpha+1}$ satisfy Hypothesis 1 with $1 \leq \alpha \leq 2$ and let $s \geq 1-\frac{\alpha}{2}$ with $(s, \alpha) \neq\left(\frac{1}{2}, 1\right)$. Then the Cauchy problem associated with (1-3) is unconditionally locally well-posed in $H^{s}(\mathbb{K})$ with a maximal time of existence $T \gtrsim\left(1+\left\|u_{0}\right\|_{H^{1-\alpha / 2}}\right)^{-2(\alpha+1) /(2 \alpha-1)}$.

Remark 1.6. In the regular case (Cauchy problem in $H^{s}$ with $s>\frac{1}{2}$ ), we actually need (1-6) only for $\left|\xi_{1}\right| \wedge\left|\xi_{2}\right| \gg 1$.

Remark 1.7. Our method also works in the case $\alpha>2$. In this case we get the unconditional wellposedness in $H^{s}(\mathbb{K})$ for $s \geq 0$.

Remark 1.8. For $L_{\alpha+1}:=\partial_{x}^{3}$, we recover the unconditional LWP results for the KdV equation in $L^{2}(\mathbb{R})$ and $L^{2}(\mathbb{})$ obtained in [Zhou 1997; Babin et al. 2011], respectively.

For $L_{\alpha+1}$ with $\left.\alpha \in\right] 1,2$ [ our results on unconditional uniqueness are, to our knowledge, new for both the real line case and the periodic case.

In the limit case $(s, \alpha)=\left(\frac{1}{2}, 1\right)$ we do not succeed in proving the unconditional uniqueness result. However, our method of proof enables us to prove the well-posedness without using a gauge transform. We think that this result is also of interest since $H^{1 / 2}$ is the energy space when $\alpha=1$. It is worth noticing that, as far as we know, the available results without gauge transformation on the local well-posedness of the Benjamin-Ono equation in Sobolev spaces $H^{s}(\mathbb{R})$ were restricted to $s \geq 1$ (see [Guo et al. 2011]). Also, the well-posedness in the energy space $H^{1 / 2}$ seems to be new for the intermediate long waves equation, at least in the periodic setting.

Theorem 1.9. Let $\mathbb{K}=\mathbb{R}$ or $\mathbb{T}$ and assume $L_{\alpha+1}$ satisfies Hypothesis 1 with $\alpha=1$. Then the Cauchy problem associated with (1-3) is locally well-posed in $H^{1 / 2}(\mathbb{K})$ with a maximal time of existence $T \gtrsim$ $\left(1+\left\|u_{0}\right\|_{H^{1 / 2}}\right)^{-4}$.

Let us assume now that the symbol $p_{\alpha+1}$ satisfies, moreover,

$$
\left|p_{\alpha+1}(\xi)\right| \lesssim|\xi| \text { for }|\xi| \leq 1 \quad \text { and } \quad\left|p_{\alpha+1}(\xi)\right| \sim|\xi|^{\alpha+1} \text { for }|\xi| \geq 1 \text {. }
$$

Then it is not too hard to check that (1-3) enjoys the conservation laws

$$
\frac{d}{d t} \int_{\mathbb{K}} u^{2} d x=0 \quad \text { and } \quad \frac{d}{d t} \int_{\mathbb{K}}\left(\left|\Lambda^{\alpha / 2} u\right|^{2}+\frac{1}{3} u^{3}\right) d x=0,
$$


where $\Lambda^{\alpha / 2}$ is the space Fourier multiplier defined by

$$
\widehat{\Lambda^{\alpha / 2}} v(\xi)=\left|\frac{p_{\alpha+1}(\xi)}{\xi}\right|^{\frac{1}{2}} \hat{v}(\xi) .
$$

Combined with the embedding $H^{\alpha / 2} \hookrightarrow L^{3}$, we get an a priori bound of the $H^{\alpha / 2}$-norm of the solution. We may then iterate Theorems 1.5 and 1.9 to obtain the following corollary:

Corollary 1.10. Let $\mathbb{K}=\mathbb{R}$ or $\mathbb{T}$ and assume $L_{\alpha+1}$ satisfies Hypothesis 1 and (1-8). Then the Cauchy problem associated with (1-3) is unconditionally globally well-posed in $H^{\alpha / 2}(\mathbb{K})$ for $1<\alpha \leq 2$, and globally well-posed in $H^{1 / 2}(\mathbb{T})$ for $\alpha=1$.

Remark 1.11. The linear operators given in Remark 1.1 also satisfy assumption (1-8).

Remark 1.12. If one considers LWP and not unconditional LWP, then the best-known results for (1-1) with $1<\alpha<2$ have been obtained in [Herr et al. 2010], where the global well-posedness in $L^{2}(\mathbb{R})$ is proved by using a paradifferential gauge transformation. As far as we know, the best available results without gauge transformation are obtained in [Guo 2012], where the LWP in $H^{s}(\mathbb{R})$ with $s>2-\alpha$ is proven. This leads to a global well-posedness result only for $\alpha>\frac{4}{3}$. Therefore, even for (1-1), our results improve the local and global available well-posedness results without the use of gauge transformation on the real line. To the best of our knowledge, they are new on the one-dimensional torus, where we are not aware of any global well-posedness result.

It is well known that, taking into account some damping or dissipative effects, dissipative versions of (1-3) can be derived (see for instance [Ott and Sudan 1970; Edwin and Roberts 1986]). One quite direct application of the fact that we do not need a gauge transform to solve (1-3) is that we can easily treat the dissipative limit of dissipative versions of (1-3). Such a dissipative limit was, for example, studied for the Benjamin-Ono equation on the real line in [Guo et al. 2011; Molinet 2013].

Let us introduce the following dissipative version of (1-3):

$$
u_{t}+L_{\alpha+1} u+\varepsilon A_{\beta} u+u u_{x}=0,
$$

where $\varepsilon>0$ is a small parameter, $\beta \geq 0$ and $A_{\beta}$ is a linear operator satisfying the following hypothesis: Hypothesis 2. We assume that $A_{\beta}$ is the Fourier multiplier operator by $q_{\beta}$, where $q_{\beta}$ is a real-valued, even function, bounded on bounded intervals, satisfying: for all $0<\lambda \ll 1$ and $\xi \gg 1$,

$$
\lambda^{\beta} q_{\beta}\left(\lambda^{-1} \xi\right) \sim|\xi|^{\beta}
$$

Remark 1.13. Both the homogeneous operators $D_{x}^{\beta}$ and the nonhomogeneous operators $J_{x}^{\beta}$ satisfy Hypothesis 2.

Theorem 1.14. Let $\mathbb{K}=\mathbb{R}$ or $\mathbb{T}, 1 \leq \alpha \leq 2,0 \leq \beta \leq 1+\alpha$ and $s \geq 1-\frac{\alpha}{2}$.

(1) Then the Cauchy problem associated with (1-9) is locally well-posed in $H^{s}(\mathbb{K})$. 
(2) For $u_{0} \in H^{s}(\mathbb{K})$, let $u$ be the solution to (1-3) emanating from $u_{0}$ and let the maximal time of existence of $u$ in $H^{s}$ be $T^{*} \gtrsim\left(1+\left\|u_{0}\right\|_{H^{1-\alpha / 2}}\right)^{-2(\alpha+1) /(2 \alpha-1)}$ (note that $T^{*}$ may be infinite). Then the maximal time of existence $T_{\varepsilon}$ of the solution $u_{\varepsilon}$ to (1-9) emanating from $u_{0}$ satisfies $\liminf _{\varepsilon \rightarrow 0} T_{\varepsilon} \geq T^{*}$. Moreover, for any $0<T_{0}<T^{*}, u_{\varepsilon} \rightarrow u$ in $C\left(\left[0, T_{0}\right] ; H^{s}\right)$ as $\varepsilon \rightarrow 0$.

Remark 1.15. The constraint $\beta \leq 1+\alpha$ is clearly an artifact of the method we used. We think that it could be dropped by replacing, in some estimates, the dispersive Bourgain spaces by dispersive-dissipative Bourgain spaces that were first introduced in [Molinet and Ribaud 2002]. But, since the dissipative operators involved in wave motions are commonly of order less or equal to 2 , we do not pursue this issue.

The rest of the paper is organized as follows: In Section 2, we introduce the notations, define the function spaces and state some preliminary lemmas. In Section 3, we develop our method in the simplest case, $s>\frac{1}{2}$, while the nonregular case is treated in Section 4. Section 5 is devoted to the proof of Theorem 1.14. We conclude the paper with an Appendix explaining how to deal with the special case $(s, \alpha)=\left(\frac{1}{2}, 1\right)$.

\section{Notations, function spaces and preliminary lemmas}

2A. Notation. For any positive numbers $a$ and $b$, the notation $a \lesssim b$ means that there exists a positive constant $c$ such that $a \leq c b$. We also write $a \sim b$ when $a \lesssim b$ and $b \lesssim a$. Moreover, if $\alpha \in \mathbb{R}$, then $\alpha_{+}$ and $\alpha_{-}$will denote a number slightly greater and less than $\alpha$, respectively.

For $u=u(x, t) \in \mathscr{S}\left(\mathbb{R}^{2}\right), \mathscr{F} u=\hat{u}$ will denote its space-time Fourier transform, whereas $\mathscr{F}_{x} u=(u)^{\wedge x}$ and $\mathscr{F}_{t} u=(u)^{\wedge t}$ will denote its Fourier transform in space and in time, respectively. For $s \in \mathbb{R}$, we define the Bessel and Riesz potentials of order $-s, J_{x}^{s}$ and $D_{x}^{s}$, by

$$
J_{x}^{s} u=\mathscr{F}_{x}^{-1}\left(\left(1+|\xi|^{2}\right)^{\frac{s}{2} \mathscr{F}_{x}} u\right) \quad \text { and } \quad D_{x}^{s} u=\mathscr{F}_{x}^{-1}\left(|\xi|^{s} \mathscr{F}_{x} u\right)
$$

Throughout the paper, we fix a smooth cutoff function $\eta$ such that

$$
\eta \in C_{0}^{\infty}(\mathbb{R}), \quad 0 \leq \eta \leq 1, \quad \eta_{\left.\right|_{[-1,1]}}=1 \quad \text { and } \quad \operatorname{supp}(\eta) \subset[-2,2] .
$$

We set $\phi(\xi):=\eta(\xi)-\eta(2 \xi)$. For $l \in \mathbb{Z}$, we define

$$
\phi_{2^{l}}(\xi):=\phi\left(2^{-l} \xi\right)
$$

and, for $l \in \mathbb{N}^{*}$,

$$
\psi_{2^{l}}(\xi, \tau)=\phi_{2^{l}}\left(\tau-p_{\alpha+1}(\xi)\right)
$$

where $i p_{\alpha+1}$ is the Fourier symbol of $L_{\alpha+1}$. By convention, we also denote

$$
\psi_{1}(\xi, \tau):=\eta\left(2\left(\tau-p_{\alpha+1}(\xi)\right)\right)
$$

Any summations over capitalized variables such as $N, L, K$ or $M$ are presumed to be dyadic. Unless stated otherwise, we work with homogeneous dyadic decomposition for the space-frequency variables 
and nonhomogeneous decompositions for modulation variables, i.e., these variables range over numbers of the form $\left\{2^{k}: k \in \mathbb{Z}\right\}$ and $\left\{2^{k}: k \in \mathbb{N}\right\}$, respectively. Then we have that

$$
\sum_{N>0} \phi_{N}(\xi)=1 \quad \text { for all } \xi \in \mathbb{R}^{*} \quad \text { and } \quad \operatorname{supp}\left(\phi_{N}\right) \subset\left\{\frac{1}{2} N \leq|\xi| \leq 2 N\right\} \quad \text { for } N \in\left\{2^{k}: k \in \mathbb{Z}\right\}
$$

and

$$
\sum_{L \geq 1} \psi_{L}(\xi, \tau)=1 \text { for all }(\xi, \tau) \in \mathbb{R}^{2}
$$

Let us define the Littlewood-Paley multipliers by

$$
P_{N} u=\mathscr{F}_{x}^{-1}\left(\phi_{N} \mathscr{F}_{x} u\right), \quad Q_{L} u=\mathscr{F}^{-1}\left(\psi_{L} \mathscr{F} u\right),
$$

$P_{\geq N}:=\sum_{K \geq N} P_{K}, P_{\leq N}:=\sum_{K \leq N} P_{K}, Q_{\geq L}:=\sum_{K \geq L} Q_{K}$ and $Q_{\leq L}:=\sum_{K \leq L} Q_{K}$. For brevity we also write $u_{N}=P_{N} u, u_{\leq N}=P_{\leq N} u$, etc.

Let $\chi$ be a (possibly complex-valued) bounded measurable function on $\mathbb{R}^{2}$ and define the pseudoproduct operator $\Pi=\Pi_{\chi}$ on $\mathscr{S}(\mathbb{R})^{2}$ by

$$
\mathscr{F}(\Pi(f, g))(\xi)=\int_{\mathbb{R}} \hat{f}\left(\xi_{1}\right) \hat{g}\left(\xi-\xi_{1}\right) \chi\left(\xi, \xi_{1}\right) d \xi_{1} .
$$

Throughout the paper, we write $\Pi=\Pi_{\chi}$, where $\chi$ may be different at each occurrence of $\Pi$. This bilinear operator behaves like a product in the sense that it satisfies the following properties:

$$
\begin{gathered}
\Pi(f, g)=f g \quad \text { if } \chi \equiv 1 \\
\int_{\mathbb{R}} \Pi_{\chi}(f, g) h=\int_{\mathbb{R}} f \Pi_{\chi_{1}}(g, h)=\int_{\mathbb{R}} \Pi_{\chi_{2}}(f, h) g
\end{gathered}
$$

with $\chi_{1}\left(\theta, \theta_{1}\right)=\bar{\chi}\left(\theta_{1}, \theta\right)$ and $\chi_{2}\left(\theta, \theta_{1}\right)=\bar{\chi}\left(\theta-\theta_{1}, \theta\right)$ for any real-valued functions $f, g, h \in \mathscr{Y}(\mathbb{R})$. Moreover, we easily check from the Bernstein inequality that, if $f_{i} \in L^{2}(\mathbb{R})$ has a Fourier transform localized in an annulus $\left\{|\xi| \sim N_{i}\right\}, i=1,2,3$, then

$$
\left|\int_{\mathbb{R}} \Pi\left(f_{1}, f_{2}\right) f_{3}\right| \lesssim N_{\min }^{\frac{1}{2}} \prod_{i=1}^{3}\left\|f_{i}\right\|_{L^{2}}
$$

where the implicit constant only depends on $\|\chi\|_{L^{\infty}\left(\mathbb{R}^{2}\right)}$ and $N_{\min }=\min \left\{N_{1}, N_{2}, N_{3}\right\}$. With this notation in hand, we will be able to systematically estimate terms of the form

$$
\int_{\mathbb{R}} P_{N}\left(u^{2}\right) \partial_{x} P_{N} u
$$

to put the derivative on the lowest frequency factor.

2B. Function spaces. For $1 \leq p \leq \infty, L^{p}(\mathbb{R})$ is the usual Lebesgue space with the norm $\|\cdot\|_{L^{p}}$ and, for $s \in \mathbb{R}, H^{s}(\mathbb{R})$ is the usual Sobolev space with its usual norm,

$$
\|\phi\|_{H^{s}}=\left\|J_{x}^{s} \phi\right\|_{L^{2}}
$$


If $B$ is one of the spaces defined above, $1 \leq p \leq \infty$, we will define the space-time spaces $L_{t}^{p} B$ and $\widetilde{L}_{t}^{p} B$ equipped with the norms

$$
\|f\|_{L_{t}^{p} B}=\left(\int_{\mathbb{R}}\|f(\cdot, t)\|_{B}^{p} d t\right)^{\frac{1}{p}},
$$

with obvious modifications for $p=\infty$, and

$$
\|f\|_{\tilde{L}_{t}^{p} B}=\left(\sum_{N>0}\left\|P_{N} f\right\|_{L_{t}^{p} B}^{2}\right)^{\frac{1}{2}} .
$$

For $s, b \in \mathbb{R}$, we introduce the Bourgain spaces $X^{s, b}$ related to the linear part of (1-3) as the completion of the Schwartz space $\mathscr{S}\left(\mathbb{R}^{2}\right)$ under the norm

$$
\|v\|_{X^{s, b}}:=\left(\int_{\mathbb{R}^{2}}\left\langle\tau-p_{\alpha+1}(\xi)\right\rangle^{2 b}\langle\xi\rangle^{2 s}|\hat{v}(\xi, \tau)|^{2} d \xi d \tau\right)^{\frac{1}{2}},
$$

where $\langle x\rangle:=1+|x|$ and $i p_{\alpha+1}$ is the Fourier symbol of $L_{\alpha+1}$. Recall that

$$
\|v\|_{X^{s, b}}=\left\|U_{\alpha}(-t) v\right\|_{H_{t, x}^{s, b}}
$$

where $U_{\alpha}(t)=\exp \left(t L_{\alpha+1}\right)$ is the generator of the free evolution associated with (1-3).

Finally, we will use restriction-in-time versions of these spaces. Let $T>0$ be a positive time and let $Y$ be a normed space of space-time functions. The restriction space $Y_{T}$ will be the space of functions $v: \mathbb{R} \times] 0, T[\rightarrow \mathbb{R}$ satisfying

$$
\|v\|_{Y_{T}}:=\inf \left\{\|\tilde{v}\|_{Y}|\tilde{v}: \mathbb{R} \times \mathbb{R} \rightarrow \mathbb{R}, \tilde{v}|_{\mathbb{R} \times] 0, T[}=v\right\}<\infty .
$$

\section{C. Preliminary lemmas.}

Lemma 2.1. Let $p: \mathbb{R} \rightarrow \mathbb{R}$ be an odd function belonging to $C^{1}(\mathbb{R}) \cap C^{2}\left(\mathbb{R}^{*}\right)$. Assume that there exist $\alpha>0$ and $\xi_{0}>0$ such that, for all $\xi \geq \xi_{0}$,

$$
\left|p^{\prime}(\xi)\right| \sim|\xi|^{\alpha} \text { and }\left|p^{\prime \prime}(\xi)\right| \sim|\xi|^{\alpha-1} .
$$

Then the Fourier multiplier $L_{\alpha+1}$ by ip satisfies Hypothesis 1 .

Proof. Let $0<\lambda \leq \xi_{0}^{-1}$ be a real number. First, by the mean value theorem, for $\xi \geq 1$,

$$
\left|p\left(\lambda^{-1} \xi\right)\right| \lesssim\left|p\left(\xi_{0}\right)\right|+\lambda^{-(\alpha+1)} \xi^{\alpha+1} \lesssim \lambda^{-1}\left(\lambda \xi_{0}\right) \max _{\xi \in\left[0, \xi_{0}\right]}\left|p^{\prime}(\xi)\right|+\xi^{\alpha+1}
$$

and thus

$$
\lambda^{\alpha+1}\left|p\left(\lambda^{-1} \xi\right)\right| \lesssim \lambda^{\alpha} \max _{\xi \in\left[0, \xi_{0}\right]}\left|p^{\prime}(\xi)\right|+\xi^{\alpha+1} \lesssim \xi^{\alpha+1}
$$

as soon as $\lambda \leq\left(\max _{\xi \in\left[0, \xi_{0}\right]}\left|p^{\prime}(\xi)\right|\right)^{-1 / \alpha}$. This proves (1-5) for

$$
\lambda_{0}=\min \left(\xi_{0}^{-1},\left(\max _{\xi \in\left[0, \xi_{0}\right]}\left|p^{\prime}(\xi)\right|\right)^{-\frac{1}{\alpha}}\right) .
$$


Let us now prove (1-6). In the sequel, we take $0<\lambda \leq \lambda_{0}$ with $\lambda_{0}$ defined by (2-5). By symmetry, we can assume $\left|\xi_{2}\right| \leq\left|\xi_{1}\right|$. We separate different cases:

Case 1: $\left|\xi_{2}\right| \ll\left|\xi_{1}\right|$. Since, by hypothesis, $\left|\xi_{1}\right| \gg 1$, it follows that $\lambda^{-1}\left|\xi_{1}\right| \gg \xi_{0}$ and thus there exists $\theta \in\left[\xi_{1}, \xi_{1}+\xi_{2}\right]$ such that

$$
\lambda^{\alpha+1}\left|p\left(\lambda^{-1}\left(\xi_{1}+\xi_{2}\right)\right)-p\left(\lambda^{-1} \xi_{1}\right)\right|=\lambda^{\alpha}\left|\xi_{2}\right|\left|p^{\prime}\left(\lambda^{-1} \theta\right)\right| \sim \lambda^{\alpha}\left|\xi_{2}\right|\left|\lambda^{-1} \theta\right|^{\alpha} \sim\left|\xi_{2}\right|\left|\xi_{1}\right|^{\alpha} .
$$

Now, if $\lambda^{-1}\left|\xi_{2}\right| \leq \xi_{0}$ then

$$
\lambda^{\alpha+1}\left|p\left(\lambda^{-1} \xi_{2}\right)\right| \leq \lambda^{\alpha}\left|\xi_{2}\right| \max _{\xi \in\left[0, \xi_{0}\right]}\left|p^{\prime}(\xi)\right| \ll\left|\xi_{2}\right|\left|\xi_{1}\right|^{\alpha}
$$

On the other hand, if $\lambda^{-1}\left|\xi_{2}\right| \geq \xi_{0}$ then

$$
\begin{aligned}
\lambda^{\alpha+1}\left|p\left(\lambda^{-1} \xi_{2}\right)\right| & =\lambda^{\alpha+1}\left|p\left(\xi_{0}\right)+p\left(\lambda^{-1} \xi_{2}\right)-p\left(\xi_{0}\right)\right| \\
& \leq \lambda^{\alpha+1}\left(\left|\xi_{0}\right| \max _{\xi \in\left[0, \xi_{0}\right]}\left|p^{\prime}(\xi)\right|+\lambda^{-1}\left|\xi_{2}\right|\left|\lambda^{-1} \xi_{2}\right|^{\alpha}\right) \\
& \leq\left|\xi_{2}\right|^{\alpha+1}+\lambda^{\alpha}\left|\xi_{2}\right| \max _{\xi \in\left[0, \xi_{0}\right]}\left|p^{\prime}(\xi)\right| \ll\left|\xi_{2}\right|\left|\xi_{1}\right|^{\alpha} .
\end{aligned}
$$

Gathering these two estimates leads to

$$
\lambda^{\alpha+1}\left|\Omega\left(\lambda^{-1} \xi_{1}, \lambda^{-1} \xi_{2}\right)\right| \sim\left|\xi_{2}\right|\left|\xi_{1}\right|^{\alpha}
$$

Case 2: $\left|\xi_{2}\right| \gtrsim\left|\xi_{1}\right|$. In this case we have $\lambda^{-1}\left|\xi_{2}\right| \gg \xi_{0}$. Since $p$ is an odd function, by symmetry we can assume that $\xi_{1}>0$.

Case 2(a): $\xi_{1} \xi_{2} \geq 0$. Then we have $0<\xi_{0} \ll \lambda^{-1} \xi_{2} \leq \lambda^{-1} \xi_{1}<\lambda^{-1} \xi_{1}+\xi_{2}$. We notice that $\lambda^{\alpha+1}\left|\Omega\left(\lambda^{-1} \xi_{1}, \lambda^{-1} \xi_{2}\right)\right|$

$$
=\lambda^{\alpha+1} \int_{\xi_{0}}^{\lambda^{-1} \xi_{2}}\left(p^{\prime}\left(\lambda^{-1} \xi_{1}+\theta\right)-p^{\prime}(\theta)\right) d \theta+\lambda^{\alpha+1}\left(p\left(\lambda^{-1} \xi_{1}+\xi_{0}\right)-p\left(\lambda^{-1} \xi_{1}\right)\right)-\lambda^{\alpha+1} p\left(\xi_{0}\right)
$$

with

$$
\left|p\left(\lambda^{-1} \xi_{1}+\xi_{0}\right)-p\left(\lambda^{-1} \xi_{1}\right)\right| \lesssim \xi_{0} \lambda^{-\alpha} \xi_{1}^{\alpha} \ll \lambda^{-\alpha-1} \xi_{2} \xi_{1}^{\alpha}
$$

and

$$
p^{\prime}\left(\lambda^{-1} \xi_{1}+\theta\right)-p^{\prime}(\theta)=\int_{0}^{\lambda^{-1} \xi_{1}} p^{\prime \prime}(\theta+\mu) d \mu
$$

But, for $\theta \geq \xi_{0}, p^{\prime \prime}$ does not change sign since $\left|p^{\prime \prime}(\theta)\right| \sim|\theta|^{\alpha-1}$ and $p^{\prime \prime}$ is continuous outside 0 . Therefore, for $\theta \in\left[\xi_{0}, \lambda^{-1} \xi_{2}\right]$, we get

$$
\int_{0}^{\lambda^{-1} \xi_{1}} p^{\prime \prime}(\theta+\mu) d \mu \sim \int_{0}^{\lambda^{-1} \xi_{1}}(\theta+\mu)^{\alpha-1} d \mu \sim\left(\left(\lambda^{-1} \xi_{1}+\theta\right)^{\alpha}-\theta^{\alpha}\right) \sim \lambda^{-\alpha} \xi_{1}^{\alpha} .
$$

Gathering these estimates we obtain

$$
\lambda^{\alpha+1}\left|\Omega\left(\lambda^{-1} \xi_{1}, \lambda^{-1} \xi_{2}\right)\right| \sim \xi_{2} \xi_{1}^{\alpha} .
$$


Case 2(b): $\xi_{1} \xi_{2}<0$. For $\xi_{1}+\xi_{2} \ll-\xi_{2}$, recalling that $p$ is an odd function, we can argue exactly as in Case 1 , but with $\xi_{1}+\xi_{2},-\xi_{2}$ and $\xi_{1}$ playing the role of $\xi_{2}, \xi_{1}$ and $\xi_{1}+\xi_{2}$, respectively. Finally, for $\xi_{1}+\xi_{2} \gtrsim-\xi_{2}$, we argue exactly as in Case 2(a) with the same exchange of roles as above.

Lemma 2.2. Assume that $p_{\alpha+1}$ satisfies (1-6) with $\lambda=1$. Let $L_{1}, L_{2}, L_{3} \geq 1,0<N_{1} \leq N_{2} \leq N_{3}$ be dyadic numbers and $u, v, w \in \mathscr{S}^{\prime}\left(\mathbb{R}^{2}\right)$. Then

$$
\int_{\mathbb{R}^{2}} \Pi\left(Q_{L_{1}} P_{N_{1}} u, Q_{L_{2}} P_{N_{2}} v\right) Q_{L_{3}} P_{N_{3}} w=0
$$

whenever the following relation is not satisfied:

$$
L_{\max } \sim N_{1} N_{2}^{\alpha} \quad \text { or } \quad\left(L_{\max } \gg N_{1} N_{2}^{\alpha} \quad \text { and } \quad L_{\max } \sim L_{\text {med }}\right),
$$

where $L_{\max }=\max \left(L_{1}, L_{2}, L_{3}\right), L_{\text {med }}=\max \left(\left\{L_{1}, L_{2}, L_{3}\right\}-\left\{L_{\max }\right\}\right)$ and where the two first implicit constants in (2-6) are related to the implicit constant in (1-6).

Proof. This is a direct consequence of the hypothesis (1-6) on the resonance function $\Omega\left(\xi_{1}, \xi_{2}\right)$, since

$$
\Omega\left(\xi_{1}, \xi_{2}\right)=\sigma\left(\tau_{1}+\tau_{2}, \xi_{1}+\xi_{2}\right)-\sigma\left(\tau_{1}, \xi_{1}\right)-\sigma\left(\tau_{2}, \xi_{2}\right)
$$

with $\sigma(\tau, \xi):=\tau-p_{\alpha+1}(\xi)$.

Lemma 2.3. Let $L \geq 1,1 \leq p \leq \infty$ and $s \in \mathbb{R}$. The operator $Q_{\leq L}$ is bounded in $L_{t}^{p} H^{s}$ uniformly in $L \geq 1$.

Proof. Let $R_{\leq L}$ be the Fourier multiplier by $\eta(\tau / L)$, where $\eta$ is as defined in Section $2 \mathrm{~A}$. The trick is to notice that $Q_{\leq L} u=U_{\alpha}(t)\left(R_{\leq L} U_{\alpha}(-t) u\right)$. Therefore, using the unitarity of $U_{\alpha}(\cdot)$ in $H^{s}(\mathbb{R})$, we infer that

$$
\begin{aligned}
\left\|Q_{\leq L} u\right\|_{L_{t}^{p} H^{s}}=\left\|U_{\alpha}(t)\left(R_{\leq L} U_{\alpha}(-t) u\right)\right\|_{L_{t}^{p} H^{s}}=\left\|R_{\leq L} U_{\alpha}(-t) u\right\|_{L_{t}^{p} H^{s}} \lesssim\left\|U_{\alpha}(-t) u\right\|_{L_{t}^{p} H^{s}} \\
=\|u\|_{L_{t}^{p} H^{s}}
\end{aligned}
$$

For any $T>0$, we consider $1_{T}$, the characteristic function of $[0, T]$, and use the decomposition

$$
1_{T}=1_{T, R}^{\mathrm{low}}+1_{T, R}^{\mathrm{high}}, \quad \widehat{1_{T, R}^{\mathrm{low}}}(\tau)=\eta\left(\frac{\tau}{R}\right) \widehat{1_{T}}(\tau)
$$

for some $R>0$.

The properties of this decomposition we will need are listed in the following lemmas.

Lemma 2.4. For any $R>0$ and $T>0$,

$$
\left\|1_{T, R}^{\text {high }}\right\|_{L^{1}} \lesssim T \wedge R^{-1}
$$

and

$$
\left\|1_{T, R}^{\text {low }}\right\|_{L^{\infty}} \lesssim 1
$$


Proof. A direct computation provides

$$
\begin{aligned}
\left\|1_{T, R}^{\mathrm{high}}\right\|_{L^{1}} & =\int_{\mathbb{R}}\left|\int_{\mathbb{R}}\left(1_{T}(t)-1_{T}\left(t-\frac{s}{R}\right)\right) \mathscr{F}^{-1} \eta(s) d s\right| d t \\
& \leq \int_{\mathbb{R}} \int_{[0, T] \backslash[s / R, T+s / R] \cup[s / R, T+s / R] \backslash[0, T]}\left|\mathscr{F}^{-1} \eta(s)\right| d t d s \\
& \lesssim \int_{\mathbb{R}}\left(T \wedge \frac{|s|}{R}\right)\left|\mathscr{F}^{-1} \eta(s)\right| d s \\
& \lesssim T \wedge R^{-1} .
\end{aligned}
$$

Finally, the proof of (2-9) follows directly from the definition of $1_{T, R}^{\text {low }}$ and Young's inequality.

Lemma 2.5. Let $u \in L^{2}\left(\mathbb{R}^{2}\right)$. Then, for any $T>0, R>0$ and $L \gg R$,

$$
\left\|Q_{L}\left(1_{T, R}^{\text {low }} u\right)\right\|_{L^{2}} \lesssim\left\|Q_{\sim L} u\right\|_{L^{2}} .
$$

Proof. By Plancherel we get

$$
\begin{aligned}
I_{L} & =\left\|Q_{L}\left(1_{T, R}^{\text {low }} u\right)\right\|_{L^{2}} \\
& =\left\|\varphi_{L}(\tau-\omega(\xi)) \widehat{1_{T, R}^{\text {low }}} *_{\tau} \hat{u}(\tau, \xi)\right\|_{L^{2}} \\
& =\left\|\sum_{L_{1} \geq 1} \varphi_{L}(\tau-\omega(\xi)) \int_{\mathbb{R}} \varphi_{L_{1}}\left(\tau^{\prime}-\omega(\xi)\right) \hat{u}\left(\tau^{\prime}, \xi\right) \eta\left(\frac{\tau-\tau^{\prime}}{R}\right) \frac{e^{-i T\left(\tau-\tau^{\prime}\right)}-1}{\tau-\tau^{\prime}} d \tau^{\prime}\right\|_{L^{2}} .
\end{aligned}
$$

In the region where $L_{1} \ll L$ or $L_{1} \gg L$, we have $\left|\tau-\tau^{\prime}\right| \sim L \vee L_{1} \gg R$, thus $I_{L}$ vanishes. On the other hand, for $L \sim L_{1}$, we get

$$
I_{L} \lesssim \sum_{L \sim L_{1}}\left\|Q_{L}\left(1_{T, R}^{\text {low }} Q_{L_{1}} u\right)\right\|_{L^{2}} \lesssim\left\|Q_{\sim L} u\right\|_{L^{2}}
$$

\section{Unconditional well-posedness in the regular case $s>\frac{1}{2}$}

In this section we develop our method in the regular case $s>\frac{1}{2}$. This will emphasize the simplicity of this approach to prove unconditional well-posedness for (1-3) posed on $\mathbb{R}$ or $\mathbb{T}$.

Let $\lambda>0$ and $L_{\alpha+1}^{\lambda}$ be the Fourier multiplier by $i \lambda^{\alpha+1} p_{\alpha+1}\left(\lambda^{-1} \cdot\right)$. We notice that if $u$ is a solution to (1-3) on ]0, $T$ [ then $u_{\lambda}(t, x)=\lambda^{\alpha} u\left(\lambda^{\alpha+1} t, \lambda x\right)$ is a solution to (1-3) on ]0, $\lambda^{-(\alpha+1)} T$ [ with $L_{\alpha+1}$ replaced by $L_{\alpha+1}^{\lambda}$. Therefore, up to this change of unknown and equation, we can always assume that the operator $L_{\alpha+1}$ verifies (1-6) with $0<\lambda \leq 1$.

3A. A priori estimates. For $s \in \mathbb{R}$ we define the function space $M^{s}$ as $M^{s}:=L_{t}^{\infty} H^{s} \cap X^{s-1,1}$, endowed with the natural norm

$$
\|u\|_{M^{s}}=\|u\|_{L_{t}^{\infty} H^{s}}+\|u\|_{X^{s-1,1}} .
$$

For $u_{0} \in H^{s}(\mathbb{R}), s>\frac{1}{2}$, we will construct a solution to (1-3) in $M_{T}^{s}$, whereas the difference of two solutions emanating from initial data belonging to $H^{s}(\mathbb{R})$ will take place in $M_{T}^{s-1}$. 
Lemma 3.1. Let $0<T<2, s>\frac{1}{2}$ and let $u \in L_{T}^{\infty} H^{s}$ be a solution to (1-3) associated with an initial datum $u_{0} \in H^{s}(\mathbb{R})$. Then $u \in M_{T}^{s}$ and

$$
\|u\|_{M_{T}^{s}} \lesssim\|u\|_{L_{T}^{\infty} H^{s}}+\|u\|_{L_{T}^{\infty} H^{s}}\|u\|_{L_{T}^{\infty} H^{\frac{1}{2}+}} \cdot
$$

Moreover, for any pair $(u, v) \in\left(L_{T}^{\infty} H^{s}\right)^{2}$ of solutions to (1-3) associated with a pair of initial data $\left(u_{0}, v_{0}\right) \in\left(H^{s}(\mathbb{R})\right)^{2}$ and any $s-1 \leq r \leq s$,

$$
\|u-v\|_{M_{T}^{r}} \lesssim\|u-v\|_{L_{T}^{\infty} H^{r}}+\|u+v\|_{L_{T}^{\infty} H^{s}}\|u-v\|_{L_{T}^{\infty} H^{r}} .
$$

Proof. We have to extend the function $u$ from $(0, T)$ to $\mathbb{R}$. For this we follow [Masmoudi and Nakanishi 2005] and introduce the extension operator $\rho_{T}$ defined by

$$
\rho_{T} u(t):=\eta(t) u\left(T \mu\left(\frac{t}{T}\right)\right),
$$

where $\eta$ is the smooth cut-off function defined in Section $2 \mathrm{~A}$ and $\mu(t)=\max (1-|t-1|, 0)$. This $\rho_{T}$ is a bounded operator from $X_{T}^{\theta, b}$ into $X^{\theta, b}$ and from $L^{p}(0, T ; X)$ into $L^{p}(\mathbb{R} ; X)$ for any $\left.\left.b \in\right]-\infty, 1\right]$, $s \in \mathbb{R}, p \in[1, \infty]$ and any Banach space $X$. Moreover, these bounds are uniform for $0<T<1$.

By using this extension operator, it is clear that we only have to estimate the $X_{T}^{s-1,1}$-norm of $u$ to prove (3-1). As noticed in Remark 1.3, $u$ satisfies the Duhamel formula of (1-3) and $u \in C\left([0, T] ; H^{\theta}\right)$ for any $\theta<s$. Hence, standard linear estimates in Bourgain's spaces lead to

$$
\begin{aligned}
\|u\|_{X_{T}^{s-1,1}} \lesssim\left\|u_{0}\right\|_{H^{s-1}}+\left\|\partial_{x}\left(u^{2}\right)\right\|_{X_{T}^{s-1,0}} & \lesssim\left\|u_{0}\right\|_{H^{s-1}}+\left\|u^{2}\right\|_{L_{T}^{2} H^{s}} \\
& \lesssim\|u\|_{L_{T}^{\infty} H^{s-1}}+\|u\|_{L_{T}^{\infty} H^{\frac{1}{2}+}}\|u\|_{L_{T}^{\infty} H^{s}}
\end{aligned}
$$

by standard product estimates in Sobolev spaces (see [Adams 1975]).

In the same way, for $s-1 \leq r \leq s$ we have

$\|u-v\|_{X_{T}^{r-1,1}} \lesssim\left\|u_{0}-v_{0}\right\|_{H^{r-1}}+\|(u+v)(u-v)\|_{L_{T}^{2} H^{r}} \lesssim\|u-v\|_{L_{T}^{\infty} H^{r-1}}+\|u+v\|_{L_{T}^{\infty} H^{s}}\|u-v\|_{L_{T}^{\infty} H^{r}}$, since $s>\frac{1}{2}+$ and $r+s>0$. This proves (3-2).

Lemma 3.2. Assume $u_{i} \in M^{0}, i=1,2,3$, are functions with spatial Fourier support in $\left\{|\xi| \sim N_{i}\right\}$ with $N_{i}>0$ dyadic satisfying $N_{1} \leq N_{2} \leq N_{3}$. For any $t>0$, we set

If $N_{1} \lesssim 1$,

$$
I_{t}\left(u_{1}, u_{2}, u_{3}\right)=\int_{0}^{t} \int_{\mathbb{R}} \Pi\left(u_{1}, u_{2}\right) u_{3} .
$$

$$
\left|I_{t}\left(u_{1}, u_{2}, u_{3}\right)\right| \lesssim N_{1}^{\frac{1}{2}}\left\|u_{1}\right\|_{L_{t}^{\infty} L_{x}^{2}}\left\|u_{2}\right\|_{L_{t x}^{2}}\left\|u_{3}\right\|_{L_{t x}^{2}} .
$$

In the case $N_{1} \gg 1$,

$$
\begin{aligned}
\left|I_{t}\left(u_{1}, u_{2}, u_{3}\right)\right| & \lesssim N_{1}^{-\frac{1}{2}} N_{3}^{1-\alpha}\left\|u_{1}\right\|_{L_{t}^{\infty} L_{x}^{2}}\left(\left\|u_{2}\right\|_{L_{t x}^{2}}\left\|u_{3}\right\|_{X^{-1,1}}+\left\|u_{2}\right\|_{X^{-1,1}}\left\|u_{3}\right\|_{L_{t x}^{2}}\right) \\
+ & N_{1}^{\frac{1}{2}} N_{3}^{-\alpha}\left\|u_{1}\right\|_{X^{-1,1}}\left\|u_{2}\right\|_{L_{t x}^{2}}\left\|u_{3}\right\|_{L_{t}^{\infty} L_{x}^{2}}+N_{1}^{-1} N_{3}^{-\frac{1}{8}}\left\|u_{1}\right\|_{L_{t}^{\infty} L_{x}^{2}}\left\|u_{2}\right\|_{L_{t}^{\infty} L_{x}^{2}}\left\|u_{3}\right\|_{L_{t}^{\infty} L_{x}^{2}} .
\end{aligned}
$$


Proof. Estimate (3-4) easily follows from (2-2) together with Hölder's inequality, thus it suffices to estimate $\left|I_{t}\right|$ for $N_{1} \gg 1$. Note that $I_{t}$ vanishes unless $N_{2} \sim N_{3}$. Setting $R=N_{1}^{3 / 2} N_{3}^{1 / 8}$, we split $I_{t}$ as

$$
I_{t}\left(u_{1}, u_{2}, u_{3}\right)=I_{\infty}\left(1_{t, R}^{\mathrm{high}} u_{1}, u_{2}, u_{3}\right)+I_{\infty}\left(1_{t, R}^{\text {low }} u_{1}, u_{2}, u_{3}\right):=I_{t}^{\mathrm{high}}+I_{t}^{\mathrm{low}},
$$

where $I_{\infty}(u, v, w)=\int_{\mathbb{R}^{2}} \Pi(u, v) w$. The contribution of $I_{t}^{\text {high }}$ is estimated, thanks to Lemma 2.4 as well as (2-2) and Hölder's inequality, by

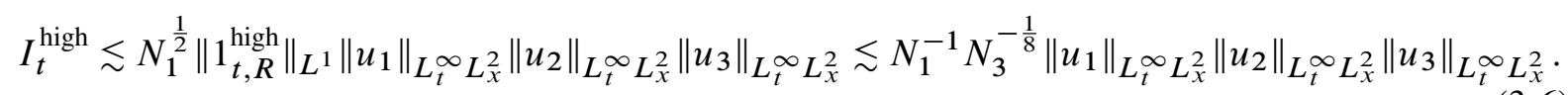

To evaluate the contribution $I_{t}^{\text {low }}$ we use that, according to Lemma 2.2, we have the decomposition

$$
\begin{aligned}
& I_{\infty}\left(1_{t, R}^{\text {low }} u_{1}, u_{2}, u_{3}\right)=I_{\infty}(\left.Q_{\gtrsim} N_{1} N_{3}^{\alpha}\left(1_{t, R}^{\text {low }} u_{1}\right), u_{2}, u_{3}\right) \\
&+I_{\infty}\left(Q_{\ll N_{1} N_{2}^{\alpha}}\left(1_{t, R}^{\text {low }} u_{1}\right), Q_{\gtrsim N_{1} N_{3}^{\alpha} u_{2}, u_{3}}\right) \\
& \quad+I_{\infty}\left(Q_{\ll N_{1} N_{2}^{\alpha}}\left(1_{t, R}^{\text {low }} u_{1}\right), Q_{\ll N_{1} N_{3}^{\alpha}} u_{2}, Q_{\sim N_{1} N_{3}^{\alpha}} u_{3}\right) .
\end{aligned}
$$

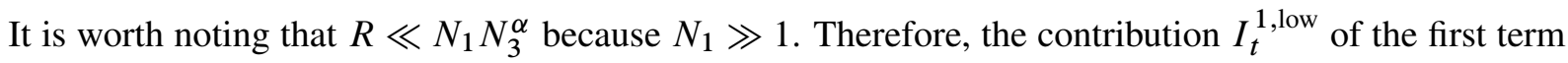
of the above right-hand side to $I_{t}^{\text {low }}$ is easily estimated, thanks to Lemma 2.5 , by

$$
I_{t}^{1, \text { low }} \lesssim N_{1}^{\frac{1}{2}}\left(N_{1} N_{3}^{\alpha}\right)^{-1}\left\|u_{1}\right\|_{X^{0,1}}\left\|u_{2}\right\|_{L_{t x}^{2}}\left\|u_{3}\right\|_{L_{t}^{\infty} L_{x}^{2}} \lesssim N_{1}^{\frac{1}{2}} N_{3}^{-\alpha}\left\|u_{1}\right\|_{X^{-1,1}}\left\|u_{2}\right\|_{L_{t x}^{2}}\left\|u_{3}\right\|_{L_{t}^{\infty} L_{x}^{2}}
$$

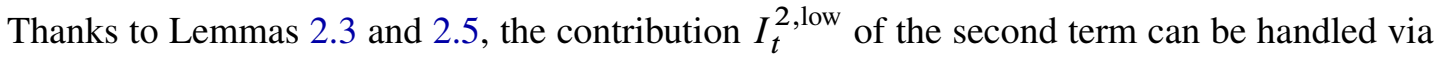

$$
\begin{aligned}
I_{t}^{2, \text { low }} & \lesssim N_{1}^{\frac{1}{2}}\left(N_{1} N_{3}^{\alpha}\right)^{-1}\left\|u_{1}\right\|_{L_{t}^{\infty} L_{x}^{2}}\left\|u_{2}\right\|_{X^{0,1}}\left\|u_{3}\right\|_{L_{t x}^{2}} \\
& \lesssim N_{1}^{-\frac{1}{2}} N_{3}^{1-\alpha}\left\|u_{1}\right\|_{L_{t}^{\infty} L_{x}^{2}}\left\|u_{2}\right\|_{X^{-1,1}}\left\|u_{3}\right\|_{L_{t x}^{2}} .
\end{aligned}
$$

Finally, the contribution of the third term is estimated in the same way.

Remark 3.3. From (2-1) we see that the estimates in Lemma 3.2 also hold for any other rearrangements of $N_{1}, N_{2}$ and $N_{3}$.

We are now in position to derive our "improved" energy estimate on smooth solutions to (1-3).

Proposition 3.4. Let $0<T<2$ and let $u \in L_{T}^{\infty} H^{s}$ with $s>\frac{1}{2}$ be a solution to (1-3) associated with an initial datum $u_{0} \in H^{s}(\mathbb{R})$. Then

$$
\|u\|_{L_{T}^{\infty} H^{s}}^{2} \lesssim\left\|u_{0}\right\|_{H^{s}}^{2}+\left(1+\|u\|_{L_{T}^{\infty} H^{\frac{1}{2}+}}^{2}\right)\|u\|_{L_{T}^{\infty} H^{\frac{1}{2}}+}\|u\|_{L_{T}^{\infty} H^{s}}^{2}
$$

Proof. We apply the operator $P_{N}$ with $N>0$ dyadic to (1-3). On account of Remark 1.3, it is clear that $P_{N} u \in C\left([0, T] ; H^{\infty}\right)$ with $\partial_{t} u_{N} \in L^{\infty}\left(0, T ; H^{\infty}\right)$. Therefore, taking the $L_{x}^{2}$-scalar product of the resulting equation with $P_{N} u$, multiplying by $\langle N\rangle^{2 s}$ and integrating on ]0, $t$ [ with $0<t<T$, we obtain

$$
\langle N\rangle^{2 s}\left\|P_{N} u(t)\right\|_{L^{2}}^{2}=\langle N\rangle^{2 s}\left\|P_{N} u_{0}\right\|_{L^{2}}^{2}+\langle N\rangle^{2 s} \int_{0}^{t} \int_{\mathbb{R}} \partial_{x} P_{N}\left(u^{2}\right) P_{N} u .
$$


Integrating by parts and applying Bernstein inequalities, this leads to

$$
\left\|P_{N} u\right\|_{L_{T}^{\infty} H^{s}}^{2} \lesssim\left\|P_{N} u_{0}\right\|_{H^{s}}^{2}+\sup _{t \in] 0, T[}\langle N\rangle^{2 s}\left|\int_{0}^{t} \int_{\mathbb{R}} P_{N}\left(u^{2}\right) \partial_{x} P_{N} u\right| .
$$

Thus it remains to estimate

$$
I:=\sum_{N>0}\langle N\rangle^{2 s} \sup _{t \in] 0, T[}\left|\int_{0}^{t} \int_{\mathbb{R}} P_{N}\left(u^{2}\right) \partial_{x} P_{N} u\right| .
$$

According to (3-1), $u$ belongs to $M_{T}^{s}$. We take an extension $\tilde{u}$ of $u$ supported in time in ]-2,2[ such that $\|\tilde{u}\|_{M^{s}} \lesssim\|u\|_{M_{T}^{s}}$. To simplify the notation we drop the tilde in the sequel.

By localization considerations, we get

$$
P_{N}\left(u^{2}\right)=P_{N}\left(u_{\gtrsim N} u_{\gtrsim N}\right)+2 P_{N}\left(u_{\ll N} u\right) .
$$

Moreover, using a Taylor expansion of $\phi_{N}$, we easily get

$$
P_{N}\left(u_{\ll N} u\right)=u_{\ll N} P_{N} u+N^{-1} \Pi\left(\partial_{x} u_{\ll N}, u\right),
$$

where $\Pi=\Pi_{\chi}$ with $\chi\left(\xi, \xi_{1}\right)=-i \int_{0}^{1} \phi^{\prime}\left(N^{-1}\left(\xi-\theta \xi_{1}\right)\right) d \theta \in L^{\infty}$. Inserting (3-13)-(3-14) into (3-12) and integrating by parts, we deduce

$$
\begin{aligned}
I \lesssim \sum_{N>0} & \sum_{0<N_{1} \ll N} N_{1}\langle N\rangle^{2 s} \sup _{t \in] 0, T[}\left|\int_{0}^{t} \int_{\mathbb{R}} \Pi_{\chi_{1}}\left(u_{N_{1}}, u_{N}\right) u_{N}\right| \\
& +\sum_{N>0} \sum_{0<N_{1} \ll N} N_{1}\langle N\rangle^{2 s} \sup _{t \in] 0, T[}\left|\int_{0}^{t} \int_{\mathbb{R}} \Pi_{\chi_{2}}\left(u_{N_{1}}, u_{\sim N}\right) u_{N}\right| \\
& +\sum_{N>0} \sum_{N_{1} \gtrsim N} N\langle N\rangle^{2 s} \sup _{t \in] 0, T[}\left|\int_{0}^{t} \int_{\mathbb{R}} \Pi_{\chi_{3}}\left(u_{N_{1}}, u_{\sim N_{1}}\right) u_{N}\right|
\end{aligned}
$$

where $\chi_{i}, 1 \leq i \leq 3$, are bounded uniformly in $N$ and $N_{1}$, and defined by

$$
\begin{aligned}
& \chi_{1}\left(\xi, \xi_{1}\right)=\frac{\xi_{1}}{N_{1}} 1_{\text {supp } \phi_{N_{1}}}\left(\xi_{1}\right), \\
& \chi_{2}\left(\xi, \xi_{1}\right)=\chi\left(\xi, \xi_{1}\right) \frac{\xi_{1}}{N_{1}} \frac{\xi}{N} \frac{1_{\text {supp } \phi_{N}}(\xi) 1_{\operatorname{supp} \phi_{N_{1}}}\left(\xi_{1}\right)}{\phi_{\sim N}\left(\xi-\xi_{1}\right)}, \\
& \chi_{3}\left(\xi, \xi_{1}\right)=\frac{\xi}{N} \phi_{N}(\xi) .
\end{aligned}
$$

Recalling now the definition of $I_{t}$ (see Lemma 3.2), it follows from (2-1) that

$$
I \lesssim \sum_{N>0} \sum_{N_{1} \gtrsim N} N\left\langle N_{1}\right\rangle^{2 s} \sup _{t \in] 0, T[}\left|I_{t}\left(u_{N}, u_{\sim N_{1}}, u_{N_{1}}\right)\right| .
$$

The contribution of the sum over $N \lesssim 1$ is easily estimated, thanks to (3-4) and Cauchy-Schwarz, by

$$
\sum_{N \leq 2^{9}} \sum_{N_{1} \gtrsim N} N\left\langle N_{1}\right\rangle^{2 s}\left\|u_{N}\right\|_{L_{t}^{\infty} L_{x}^{2}}\left\|u_{N_{1}}\right\|_{L_{t}^{2} L_{x}^{2}}^{2} \lesssim\|u\|_{L_{t}^{\infty} L_{x}^{2}}\|u\|_{L_{t}^{\infty} H^{s}}^{2} .
$$


Finally, the contribution of the sum over $N \gg 1$ is controlled with the second part of Lemma 3.2 by

$$
\begin{aligned}
& \sum_{N>2^{9}} \sum_{N_{1} \gtrsim N} N N_{1}^{2 s}[ N^{-\frac{1}{2}} N_{1}^{1-\alpha}\left\|u_{N}\right\|_{L_{t}^{\infty} L_{x}^{2}}\left\|u_{N_{1}}\right\|_{L_{t x}^{2}}\left\|u_{N_{1}}\right\|_{X^{-1,1}} \\
&\left.+N^{\frac{1}{2}} N_{1}^{-\alpha}\left\|u_{N}\right\|_{X^{-1,1}}\left\|u_{N_{1}}\right\|_{L_{t}^{\infty} L_{x}^{2}}^{2}+N^{-1} N_{1}^{-\frac{1}{8}}\left\|u_{N}\right\|_{L_{t}^{\infty} L_{x}^{2}}\left\|u_{N_{1}}\right\|_{L_{t}^{\infty} L_{x}^{2}}^{2}\right] \\
& \lesssim\|u\|_{M_{T}^{\frac{1}{2}+}\|u\|_{M_{T}^{s}}\|u\|_{L_{T}^{\infty} H^{s}} .}
\end{aligned}
$$

Gathering all the above estimates leads to

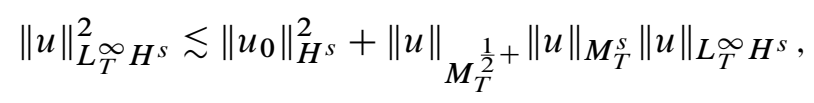

which, together with (3-1), completes the proof of the proposition.

Let us now establish an a priori estimate at the regularity level $s-1$ on the difference of two solutions.

Proposition 3.5. Let $0<T<2$ and let $u, v \in L_{T}^{\infty} H^{s}$ with $s>\frac{1}{2}$ be two solutions to (1-3) associated with initial data $u_{0}, v_{0} \in H^{s}(\mathbb{R})$, respectively. Then

$$
\|u-v\|_{L_{T}^{\infty} H^{s-1}}^{2} \lesssim\left\|u_{0}-v_{0}\right\|_{H^{s-1}}^{2}+\|u+v\|_{M_{T}^{s}}\|u-v\|_{M_{T}^{s-1}}^{2}
$$

Proof. The difference $w=u-v$ satisfies

$$
w_{t}+D^{\alpha} w_{x}=\partial_{x}(z w)
$$

where $z=u+v$. Proceeding as in the proof of Proposition 3.4, we infer that, for $N>0$,

$$
\left\|P_{N} w\right\|_{L_{T}^{\infty} H^{s-1}}^{2} \lesssim\left\|P_{N} w_{0}\right\|_{H^{s-1}}^{2}+\sup _{t \in] 0, T[}\langle N\rangle^{2(s-1)}\left|\int_{0}^{t} \int_{\mathbb{R}} P_{N}(z w) \partial_{x} P_{N} w\right|
$$

Again, according to (3-1), we can take extensions $\tilde{z}$ and $\tilde{w}$ of $z$ and $w$ supported in time in ]-2, 2[ such that $\|\tilde{z}\|_{M^{s}} \lesssim\|z\|_{M_{T}^{s}}$ and $\|\tilde{w}\|_{M^{s-1}} \lesssim\|w\|_{M_{T}^{s-1}}$. To simplify the notation we drop the tilde in the sequel.

Setting

$$
J:=\sum_{N>0}\langle N\rangle^{2(s-1)} \sup _{t \in] 0, T[}\left|\int_{0}^{t} \int_{\mathbb{R}} P_{N}(z w) \partial_{x} P_{N} w\right|
$$

it follows from (3-14) and classical dyadic decomposition that, for all $N>0$,

$$
\begin{aligned}
P_{N}(z w) & =P_{N}\left(z_{\ll N} w\right)+P_{N}\left(z_{\sim N} w_{\lesssim N}\right)+\sum_{N_{1} \gg N} P_{N}\left(z_{N_{1}} w_{\sim N_{1}}\right) \\
& =z_{\ll N} w_{N}+N^{-1} \Pi_{\chi}\left(\partial_{x} z_{\ll N}, w\right)+P_{N}\left(z_{\sim N} w_{\lesssim N}\right)+\sum_{N_{1} \gg N} P_{N}\left(z_{N_{1}} w_{\sim N_{1}}\right) .
\end{aligned}
$$


Inserting this into (3-25) and integrating by parts, we infer

$$
\begin{array}{r}
J \lesssim \sum_{N>0} \sum_{N_{1} \ll N} N_{1}\langle N\rangle^{2(s-1)}\left(\sup _{t \in] 0, T[}\left|\int_{0}^{t} \int_{\mathbb{R}} \Pi_{\chi_{1}}\left(z_{N_{1}}, w_{N}\right) w_{N}\right|+\sup _{t \in] 0, T[}\left|\int_{0}^{t} \int_{\mathbb{R}} \Pi_{\chi_{2}}\left(z_{N_{1}}, w_{\sim}\right) w_{N}\right|\right) \\
+\sum_{N>0} \sum_{N_{1} \lesssim N} N\langle N\rangle^{2(s-1)} \sup _{t \in] 0, T[}\left|\int_{0}^{t} \int_{\mathbb{R}} \Pi_{\chi_{3}}\left(z_{\sim} \sim N, w_{N_{1}}\right) w_{N}\right| \\
+\sum_{N>0} \sum_{N_{1} \gg N} N\langle N\rangle^{2(s-1)} \sup _{t \in] 0, T[}\left|\int_{0}^{t} \int_{\mathbb{R}} \Pi_{\chi_{3}}\left(z_{N_{1}}, w_{\sim N_{1}}\right) w_{N}\right|,
\end{array}
$$

where $\chi_{i}, 1 \leq i \leq 3$, are as defined in (3-15)-(3-17). Therefore, it suffices to estimate

$$
\begin{aligned}
& J \lesssim \sum_{N>0} \sum_{N_{1} \gtrsim N} N\left\langle N_{1}\right\rangle^{2(s-1)} \sup _{t \in] 0, T[}\left|I_{t}\left(z_{N}, w_{\sim} N_{1}, w_{N_{1}}\right)\right| \\
& \quad+\sum_{N>0} \sum_{N_{1} \gtrsim N} N_{1}\left\langle N_{1}\right\rangle^{2(s-1)} \sup _{t \in] 0, T[}\left|I_{t}\left(z_{\sim} N_{1}, w_{N}, w_{N_{1}}\right)\right| \\
& \quad+\sum_{N>0} \sum_{N_{1} \gtrsim N} N\langle N\rangle^{2(s-1)} \sup _{t \in] 0, T[}\left|I_{t}\left(z_{N_{1}}, w_{N_{1}}, w_{N}\right)\right| \\
& :=J_{1}+J_{2}+J_{3} .
\end{aligned}
$$

The contribution of the sum over $N \lesssim 1$ in (3-27) is easily estimated, thanks to (3-4), by

$$
\begin{array}{r}
\sum_{N \lesssim 1} \sum_{N_{1} \gtrsim N} N^{\frac{1}{2}}\left(N\left\|z_{N}\right\|_{L_{t}^{\infty} L_{x}^{2}}\left\|w_{N_{1}}\right\|_{L_{t}^{2} H^{s-1}}^{2}+N_{1}\left\langle N_{1}\right\rangle^{-1}\left\|z_{N_{1}}\right\|_{L_{t}^{2} H^{s}}\left\|w_{N}\right\|_{L_{t}^{\infty} L_{x}^{2}}\left\|w_{N_{1}}\right\|_{L_{t}^{2} H^{s-1}}\right. \\
\left.+N\left\langle N_{1}\right\rangle^{1-2 s}\left\|z_{N_{1}}\right\|_{L_{t}^{2} H^{s}}\left\|w_{N_{1}}\right\|_{L_{t}^{2} H^{s-1}}\left\|w_{N}\right\|_{L_{t}^{\infty} L_{x}^{2}}\right) \\
\lesssim\|z\|_{L_{t}^{\infty} L_{x}^{2}\|w\|_{L_{t}^{\infty} H^{s-1}}^{2}+\|w\|}\left\|_{L_{t}^{\infty} H_{x}^{-\frac{1}{2}}}\right\| z\left\|_{L_{t}^{\infty} H^{s}}\right\| w \|_{L_{t}^{\infty} H^{s-1}}
\end{array}
$$

For the contribution of the sum over $N \gg 1$, it is worth noting that, since $s>\frac{1}{2}$, the term $J_{3}$ is controlled by $J_{2}$. The contribution of $J_{1}$ is estimated, thanks to Lemma 3.2, by

$$
\begin{array}{r}
\sum_{N \gg 1} \sum_{N_{1} \gtrsim N} N N_{1}^{2(s-1)}\left[N^{-\frac{1}{2}} N_{1}^{1-\alpha}\left\|z_{N}\right\|_{L_{t}^{\infty} L_{x}^{2}}\left\|w_{N_{1}}\right\|_{L_{t x}^{2}}\left\|w_{N_{1}}\right\|_{X^{-1,1}}\right. \\
\left.+N^{\frac{1}{2}} N_{1}^{-\alpha}\left\|z_{N}\right\|_{X^{-1,1}}\left\|w_{N_{1}}\right\|_{L_{t}^{\infty} L_{x}^{2}}^{2}+N^{-1} N_{1}^{-\frac{1}{8}}\left\|z_{N}\right\|_{L_{t}^{\infty} L_{x}^{2}}\left\|w_{N_{1}}\right\|_{L_{t}^{\infty}}^{2} L_{x}^{2}\right] \\
\lesssim\|z\|_{M^{\frac{1}{2}}+}\|w\|_{M^{s-1}}\|w\|_{L_{t}^{\infty} H^{s-1}}
\end{array}
$$

Finally, in the same way we bound $J_{2}$ by

$$
\begin{gathered}
\sum_{N \gg 1} \sum_{N_{1} \gtrsim N} N_{1}^{2 s-1}\left[N^{-\frac{1}{2}} N_{1}^{1-\alpha}\left\|w_{N}\right\|_{L_{t}^{\infty} L_{x}^{2}}\left(\left\|z_{N_{1}}\right\|_{L_{t x}^{2}}\left\|w_{N_{1}}\right\|_{X^{-1,1}}+\left\|z_{N_{1}}\right\|_{X^{-1,1}}\left\|w_{N_{1}}\right\|_{L_{t x}^{2}}\right)\right. \\
+N^{\frac{1}{2}} N_{1}^{-\alpha}\left\|w_{N}\right\|_{X^{-1,1}}\left\|z_{N_{1}}\right\|_{L_{t}^{\infty} L_{x}^{2}}\left\|w_{N_{1}}\right\|_{L_{t}^{\infty} L_{x}^{2}} \\
\left.+N^{-1} N_{1}^{-\frac{1}{8}}\left\|w_{N}\right\|_{L_{t}^{\infty} L_{x}^{2}}\left\|z_{N_{1}}\right\|_{L_{t}^{\infty} L_{x}^{2}}\left\|w_{N_{1}}\right\|_{L_{t}^{\infty} L_{x}^{2}}\right] \\
\lesssim\|z\|_{M^{s}}\|w\|_{M^{-\frac{1}{2}+}}\|w\|_{L_{t}^{\infty} H^{s-1}}+\|z\|_{M^{s}}\|w\|_{M^{s-1}}\|w\|_{L_{t}^{\infty} H^{-\frac{1}{2}+}} .
\end{gathered}
$$


Gathering the estimates (3-27)-(3-30), we obtain

$$
J \lesssim\left(\|z\|_{M_{T}^{\frac{1}{2}+}}\|w\|_{M^{s-1}}+\|z\|_{M_{T}^{s}}\|w\|_{M^{-\frac{1}{2}+}}\right)\|w\|_{L_{T}^{\infty} H^{s-1}}+\|z\|_{M_{T}^{s}}\|w\|_{M^{s-1}}\|w\|_{L_{T}^{\infty} H^{-\frac{1}{2}+}},
$$

which leads to (3-22) and completes the proof of the proposition.

3B. Unconditional well-posedness. Fix $s>\frac{1}{2}$. First, it is worth noticing that we can always assume that we deal with data that have small $H^{s}$-norm. Indeed, if $u \in L^{\infty}\left(0, T ; H^{s}\right)$ is a solution to (1-3), then, for $0<\lambda \leq 1, u_{\lambda}:=\lambda^{\alpha} u\left(\lambda^{\alpha+1} \cdot \lambda \cdot\right) \in L^{\infty}\left(0, \lambda^{\alpha+1} T ; H^{s}\right)$ is a solution to (1-3) with $L_{\alpha+1}$ replaced by $L_{\alpha+1}^{\lambda}$, that is, the Fourier multiplier by $i \lambda^{\alpha+1} p_{\alpha+1}\left(\lambda^{-1} \cdot\right)$. Recall that we assumed at the beginning of this section that $L_{\alpha+1}^{\lambda}$ satisfies (1-6) for any $0<\lambda \leq 1$. For $0<\varepsilon \ll 1$, let us denote by $\mathscr{B}^{s}(\varepsilon)$ the ball of $H^{s}(\mathbb{R})$ centered at the origin with radius $\varepsilon$. Since

$$
\left\|u_{\lambda}(0)\right\|_{H^{s}} \lesssim \lambda^{\alpha-\frac{1}{2}}\left\|u_{0}\right\|_{H^{s}}
$$

we see that we can force $u_{0, \lambda}$ to belong to $\mathscr{B}^{s}(\varepsilon)$ by choosing $\lambda=\left[\varepsilon\left(1+\left\|u_{0}\right\|_{H^{s}}\right)\right]^{-1 /(\alpha-1 / 2)}$. Therefore, the unconditional well-posedness in $H^{s}(\mathbb{R})$ of (1-3) for small $H^{s}$-initial data with a time of existence $T \geq 1$ will ensure the unconditional well-posedness of (1-3) for arbitrary large $H^{s}$-initial data with a maximal time of existence

$$
T \gtrsim\left(1+\left\|u_{0}\right\|_{H^{s}}\right)^{-\frac{2(\alpha+1)}{2 \alpha-1}} .
$$

Existence and unconditional uniqueness. It is well known (see for instance [Abdelouhab et al. 1989]) that (1-3) is locally well-posed in $H^{s}$ for $s>\frac{3}{2}$ with a minimal time of existence $T=T\left(\left\|u_{0}\right\|_{H^{3 / 2+}}\right)>0$. So, let $u \in C\left(\left[0, T_{0}\right] ; H^{\infty}(\mathbb{R})\right.$ be a smooth solution to (1-3) emanating from a smooth initial datum $u_{0} \in H^{\infty}(\mathbb{R})$ with $\left\|u_{0}\right\|_{H^{s}} \ll 1$. According to (3-10),

$$
\|u\|_{L_{T}^{\infty} H^{s}}^{2} \lesssim\|u(0)\|_{H^{s}}^{2}+\left(1+\|u\|_{L_{T}^{\infty} H^{\frac{1}{2}+}}^{2}\right)\|u\|_{L_{T}^{\infty} H^{\frac{1}{2}}+\|u\|_{L_{T}^{\infty} H^{s}}^{2}}
$$

for any $0<T \leq \min \left(1, T_{0}\right)$ and $s>\frac{1}{2}$. Let us denote by $T^{*} \geq T_{0}$ the maximal time of existence of $u$ in $H^{\infty}(\mathbb{R})$. The well-posedness result in [Abdelouhab et al. 1989] ensures that $\lim _{T \nearrow T^{*}}\|u\|_{L_{T}^{\infty} H^{3}}=+\infty$ whenever $T^{*}$ is finite. Since

$$
\|u(0)\|_{H^{\frac{1}{2}}+} \leq\|u(0)\|_{H^{s}} \ll 1,
$$

(3-32) together with the continuity of $T \mapsto\|u\|_{L_{T}^{\infty} H^{1 / 2+}}$ on $] 0, T^{*}[$ ensure that

$$
\|u\|_{L_{T^{\prime}}^{\infty} H^{\frac{1}{2}}+} \lesssim\|u(0)\|_{H^{\frac{1}{2}}+} \ll 1
$$

with $T^{\prime}=\min \left(1, T^{*}\right)$. But then (3-32) leads, for any $s>\frac{1}{2}$, to

$$
\|u\|_{L_{T^{\prime}}^{\infty} H^{s}} \lesssim\|u(0)\|_{H^{s}} .
$$

This proves that $T^{\prime}<T^{*}$ and thus $T^{\prime}=1$ and $T^{*} \geq 1$.

Now, let $u_{0} \in H^{s}(\mathbb{R})$ with $s>\frac{1}{2}$. From the above estimates, we infer that we can pass to the limit on a sequence of solutions $\left\{u_{n}\right\}$ emanating from smooth approximations of $u_{0}$ to obtain the existence of a solution $u \in L_{T}^{\infty} H^{s}$ of (1-3) with initial data $u_{0}$. Note that one can easily pass to the limit on $u_{n}^{2}$ 
by compactness arguments, since $\left\{u_{n}\right\}$ and $\left\{\partial_{t} u_{n}\right\}$ are bounded in $L_{T}^{\infty} H^{s}$ and $L_{T}^{\infty} H^{s-3}$, respectively. Estimates (3-22) and (3-1)-(3-2) then ensure that this solution is the only one in this class. Now the continuity of $u$ with values in $H^{s}(\mathbb{R})$ as well as the continuity of the flow map in $H^{s}(\mathbb{R})$ will follow from the Bona-Smith argument [1975]. For any $\varphi \in H^{s}(\mathbb{R})$, dyadic integer $N \geq 1$ and $r \geq 0$, straightforward calculations in Fourier space lead to

$$
\left\|P_{\leq N} \varphi\right\|_{H_{x}^{s+r}} \lesssim N^{r}\|\varphi\|_{H_{x}^{s}} \quad \text { and } \quad\left\|\varphi-P_{\leq N} \varphi\right\|_{H_{x}^{s-r}} \lesssim N^{-r}\left\|P_{>N} \varphi\right\|_{H_{x}^{s}} .
$$

Let $u_{0} \in H^{s}$ with $s>\frac{1}{2}$ be such that $\left\|u_{0}\right\|_{H^{s}} \ll 1$. We denote by $u^{N} \in L^{\infty}\left(0,1 ; H^{s}\right)$ the solution of (1-3) emanating from $u_{0}^{N}=P_{\leq N} u_{0}$ and, for $1 \leq N_{1} \leq N_{2}$, we set

$$
w:=u^{N_{1}}-u^{N_{2}} \text {. }
$$

Then, (3-22) and (3-2) lead to

$$
\|w\|_{M_{1}^{s-1}} \lesssim\|w(0)\|_{H^{s-1}} \lesssim N_{1}^{-1}\left\|P_{>N_{1}} u_{0}\right\|_{H^{s}}
$$

Moreover, for any $r \geq 0$ and $s>\frac{1}{2}$, we have

$$
\left\|u^{N_{i}}\right\|_{M_{1}^{s+r}} \lesssim\left\|u_{0}^{N_{i}}\right\|_{H^{s+r}} \lesssim N_{i}^{r}\left\|u_{0}\right\|_{H^{s}} .
$$

Next, we observe that $w$ solves the equation

$$
w_{t}+L_{\alpha+1} w=\frac{1}{2} \partial_{x}\left(w^{2}\right)+\partial_{x}\left(u^{N_{1}} w\right) .
$$

Proposition 3.6. Let $0<T<2$ and let $w \in M_{T}^{S}$ with $s>\frac{1}{2}$ be a solution to (3-36). Then

$$
\|w\|_{L_{T}^{\infty} H^{s}}^{2} \lesssim\|w(0)\|_{H^{s}}^{2}+\|w\|_{M_{T}^{s}}^{3}+\left\|u^{N_{1}}\right\|_{M_{T}^{s}}\|w\|_{M_{T}^{s}}^{2}+\left\|u^{N_{1}}\right\|_{M_{T}^{s+1}}\|w\|_{M_{T}^{s}}\|w\|_{M_{T}^{s-1}}
$$

Proof. Actually, this is a consequence of estimates derived in the proof of Propositions 3.4 and 3.5. We separate the contributions of $\partial_{x}\left(w^{2}\right)$ and $\partial_{x}\left(u^{N_{1}} w\right)$. Let $\left.t \in\right] 0, T[$. First, (3-21) leads to

$$
\sum_{N>0} N^{2 s}\left|\int_{0}^{t} \int_{\mathbb{R}} P_{N} \partial_{x}\left(w^{2}\right) P_{N} w\right| \lesssim\|w\|_{M_{T}^{s}}^{3} .
$$

Second, applying (3-31) at the level $s$ with $z$ replaced by $u^{N_{1}}$, we obtain

$$
\sum_{N>0} N^{2 s}\left|\int_{0}^{t} \int_{\mathbb{R}} P_{N} \partial_{x}\left(u^{N_{1}} w\right) P_{N} w\right| \lesssim\left\|u^{N_{1}}\right\|_{M_{T}^{s}}\|w\|_{M_{T}^{s}}^{2}+\left\|u^{N_{1}}\right\|_{M_{T}^{s+1}}\|w\|_{M_{T}^{s}}\|w\|_{M_{T}^{-\frac{1}{2}+}},
$$

which leads to (3-37) since $s>\frac{1}{2}$.

Combining (3-2) with (3-37) and (3-35), we get

$\|w\|_{M_{1}^{s}}^{2} \lesssim\left(1+\left\|u_{0}\right\|_{H^{s}}^{2}\right)\left[\left\|w_{0}\right\|_{H^{s}}^{2}+\left\|u_{0}\right\|_{H^{s}}\|w\|_{M_{1}^{s}}^{2}+\left\|u_{0}\right\|_{H^{s}}\|w\|_{M_{1}^{s}}^{2}+N_{1}\left\|u_{0}\right\|_{H^{s}}\|w\|_{M_{1}^{s}}\|w\|_{M_{1}^{s-1}}\right]$.

Then, the smallness assumption on $\left\|u_{0}\right\|_{H^{s}}$ and (3-34) lead to

$$
\|w\|_{M_{1}^{s}}^{2} \lesssim\left\|w_{0}\right\|_{H^{s}}^{2}+N_{1}^{2}\|w\|_{M_{1}^{s-1}}^{2} \lesssim\left\|P_{>N_{1}} u_{0}\right\|_{H^{s}}^{2}\left(1+\left\|P_{>N_{1}} u_{0}\right\|_{H^{s}}^{2}\right) \rightarrow 0 \quad \text { as } N_{1} \rightarrow 0 .
$$


This shows that $\left\{u^{N}\right\}$ is a Cauchy sequence in $C\left([0,1] ; H^{s}\right)$ and thus $\left\{u^{N}\right\}$ converges in $C\left([0,1] ; H^{s}\right)$ to a solution of (1-3) emanating from $u_{0}$. Then, the uniqueness result ensures that $u \in C\left([0,1] ; H^{s}\right)$.

Continuity of the flow map. Now let $\left\{u_{0, n}\right\} \subset H^{s}(\mathbb{R})$ be such that $u_{0, n} \rightarrow u_{0}$ in $H^{s}(\mathbb{R})$. We want to prove that the emanating solution $u_{n}$ tends to $u$ in $C\left([0,1] ; H^{s}\right)$. By the triangle inequality, for $n$ large enough,

$$
\left\|u-u_{n}\right\|_{L_{1}^{\infty} H^{s}} \leq\left\|u-u^{N}\right\|_{L_{1}^{\infty} H^{s}}+\left\|u^{N}-u_{n}^{N}\right\|_{L_{1}^{\infty} H^{s}}+\left\|u_{n}^{N}-u_{n}\right\|_{L_{1}^{\infty} H^{s}} .
$$

Using the estimate (3-38) on the solution to (3-36) we first infer that

$$
\left\|u-u^{N}\right\|_{M_{1}^{s}}+\left\|u_{n}-u_{n}^{N}\right\|_{M_{1}^{s}} \lesssim\left\|P_{>N} u_{0}\right\|_{H^{s}}+\left\|P_{>N} u_{0, n}\right\|_{H^{s}}
$$

and thus

$$
\lim _{N \rightarrow \infty} \sup _{n \in \mathbb{N}}\left(\left\|u-u^{N}\right\|_{L_{1}^{\infty} H^{s}}+\left\|u_{n}-u_{n}^{N}\right\|_{L_{1}^{\infty} H^{s}}\right)=0 .
$$

Next, we notice that (3-22) and (3-2) ensure that

$$
\left\|u^{N}-u_{n}^{N}\right\|_{M_{1}^{s-1}} \lesssim\left\|u_{0}^{N}-u_{0, n}^{N}\right\|_{H^{s-1}},
$$

and thus (3-38) and (3-34) lead to

$$
\left\|u^{N}-u_{n}^{N}\right\|_{M_{1}^{s}}^{2} \lesssim\left\|u_{0}^{N}-u_{0, n}^{N}\right\|_{H^{s}}^{2}+N^{2}\left\|u_{0}^{N}-u_{0, n}^{N}\right\|_{H^{s-1}}^{2} \lesssim\left\|u_{0}-u_{0, n}\right\|_{H^{s}}^{2}\left(1+N^{2}\right) .
$$

Combining (3-39) and (3-40), we obtain the continuity of the flow map. The proof of Theorem 1.5 is thus completed in the case $\mathbb{K}=\mathbb{R}$ and $s>\frac{1}{2}$.

3C. The periodic case. In this subsection we explain the necessary adaptations to treat the periodic case. First, we define our function spaces in the periodic setting. Since the map $u \mapsto u_{\lambda}$ maps $L^{\infty}\left(0, T ; H^{s}(\mathbb{T})\right)$ into $L^{\infty}\left(0, \lambda^{\alpha+1} T ; H^{s}(\lambda \mathbb{\mathbb { T }})\right)$, we will have to consider space of functions on the tori $\lambda \mathbb{T}$ with $\lambda \geq 1$. We use the same notations as in [Colliander et al. 2004] to deal with Fourier transform of space-periodic functions with a large period $2 \pi \lambda$. Then, $(d \xi)_{\lambda}$ will be the renormalized counting measure on $\lambda^{-1} \mathbb{Z}$ :

$$
\int a(\xi)(d \xi)_{\lambda}=\frac{1}{\lambda} \sum_{\xi \in \lambda-1 \mathbb{Z}} a(\xi) \text {. }
$$

As noticed in [Colliander et al. 2004], $(d \xi)_{\lambda}$ is the counting measure on the integers when $\lambda=1$ and converges weakly to the Lebesgue measure when $\lambda \rightarrow \infty$. In the definitions below, all the Lebesgue norms in $\xi$ will be with respect to the measure $(d \xi) \lambda$. For a $2 \pi \lambda$-periodic function $\varphi$, we define its space Fourier transform on $\lambda^{-1} \mathbb{Z}$ by

$$
\hat{\varphi}(\xi)=\int_{\lambda \mathbb{T}} e^{-i \xi x} f(x) d x \quad \text { for all } \xi \in \lambda^{-1} \mathbb{Z} .
$$

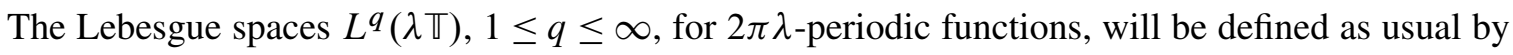

$$
\|\varphi\|_{L^{q}}=\left(\int_{\lambda \mathbb{T}}|\varphi(x)|^{q} d x\right)^{\frac{1}{q}}
$$

with the obvious modification for $q=\infty$. 
The Sobolev spaces $H^{s}(\lambda \mathbb{\mathbb { T }})$ for $2 \pi \lambda$-periodic functions are endowed with the norm

$$
\|\varphi\|_{H^{s}}=\left\|\langle\xi\rangle^{s} \hat{\varphi}(\xi)\right\|_{L_{\xi}^{2}}=\left\|J_{x}^{s} \varphi\right\|_{L^{2}}
$$

where $\langle\cdot\rangle=\left(1+|\cdot|^{2}\right)^{1 / 2}$ and $\widehat{J_{x}^{s} \varphi}(\xi)=\langle\xi\rangle^{s} \hat{\varphi}(\xi)$.

In the same way, for a function $u(t, x)$ on $\mathbb{R} \times \lambda \mathbb{T}$, we define its space-time Fourier transform by

$$
\hat{u}(\tau, \xi)=\mathscr{F}_{t, x}(u)(\tau, \xi)=\int_{\mathbb{R}} \int_{\lambda \mathbb{T}} e^{-i(\tau t+\xi x)} u(t, x) d x d t \quad \text { for all }(\tau, \xi) \in \mathbb{R} \times \lambda^{-1} \mathbb{Z} .
$$

For any $(s, b) \in \mathbb{R}^{2}$, we define the Bourgain space $X^{s, b}$ of $2 \pi \lambda$-periodic (in $x$ ) functions as the completion of $\mathscr{S}(\lambda \mathbb{\mathbb { V }} \times \mathbb{R})$ for the norm

$$
\|u\|_{X^{s, b}}=\left\|\left\langle\tau-p_{\alpha+1}(\xi)\right\rangle^{b}\langle\xi\rangle^{s} \hat{u}\right\|_{L_{\tau, \xi}^{2}} .
$$

Finally, we define the functions $\phi_{N}$ and $\psi_{L}$ and the Fourier multipliers $P_{N}$ and $Q_{L}$ as in Section 2 A. Since we take a homogeneous decomposition in space frequencies, in the periodic setting

$$
u=P_{0} u+\sum_{N>0} P_{N} u
$$

where $\widehat{P_{0} u}(\xi)=\hat{u}(0)$.

Now, with these definitions, we claim that Lemma 3.1 and Propositions 3.4, 3.5 and 3.6 also hold for $2 \pi \lambda$-periodic functions with an implicit constant that does not depend on $\lambda \geq 1$. Indeed, all the tools (the Sobolev and Hölder inequalities) we used in the proofs of these results work also in the periodic setting, independently of the period. However, in view of (3-41), we have to care about the contribution of the null-space frequencies, since we take an homogeneous decomposition. First, since the nonlinear term is a pure derivative, it is clear that the contribution of the null frequency of the nonlinear term vanishes in all the estimates. Now, it is also direct to check that

$$
\int_{\lambda \pi} P_{N}\left(u P_{0} u\right) \partial_{x} P_{N} u=0
$$

and, in the same way,

$$
\int_{\lambda \pi} P_{N}\left(w P_{0} z\right) \partial_{x} P_{N} w=0
$$

We thus just have to control the contribution of the terms $P_{N}\left(z P_{0} w\right)$ in Proposition 3.5 and $P_{N}\left(u^{N_{1}} P_{0} w\right)$ in Proposition 3.6. But the contribution of the first term in Proposition 3.5 can be easily estimated by

$$
\begin{aligned}
N^{2(s-1)}\left|\int_{0}^{t} \int_{\lambda \mathbb{T}} P_{N}\left(z P_{0} w\right) \partial_{x} P_{N} w\right| & \lesssim \sup _{\left.t^{\prime} \in\right] 0, T[}\left|\hat{w}\left(t^{\prime}, 0\right)\right| N^{2(s-1)} N\left\|P_{N} z\right\|_{L_{T}^{2} L^{2}}\left\|P_{N} w\right\|_{L_{T}^{2} L^{2}} \\
& \lesssim \delta_{N}\|z\|_{L_{T}^{\infty} H^{s}}\|w\|_{L_{T}^{\infty} H^{s-1}}^{2}
\end{aligned}
$$

where $\left\|\left(\delta_{2^{j}}\right)_{j \in \mathbb{Z}}\right\|_{l^{1}(\mathbb{Z})} \lesssim 1$. Finally, the contribution of the second term in Proposition 3.6 can be estimated in exactly the same way by

$$
N^{2 s}\left|\int_{0}^{t} \int_{\lambda \mathbb{T}} P_{N}\left(u^{N_{1}} P_{0} w\right) \partial_{x} P_{N} w\right| \lesssim \delta_{N}\left\|u^{N_{1}}\right\|_{L_{T}^{\infty} H^{s+1}}\|w\|_{L_{T}^{\infty} H^{s}}\|w\|_{L_{T}^{\infty} H^{s-1}} .
$$


This completes the proof of the regular case $s>\frac{1}{2}$ in the periodic setting.

\section{Estimates in the nonregular case}

In this section, we provide the needed estimates at level $s \geq 1-\frac{\alpha}{2}$ for $1<\alpha \leq 2$. We introduce the space

$$
F^{s, b}=F^{s, \alpha, b}=X^{s-\frac{\alpha+1}{2}, b+\frac{1}{2}}+X^{s-\frac{1+\alpha}{8}, b+\frac{1}{8}},
$$

endowed with the usual norm, and we define

$$
Y^{s}=Y^{s, \alpha}=L_{t}^{\infty} H^{s} \cap F^{s, \alpha, \frac{1}{2}}=L_{t}^{\infty} H^{s} \cap\left(X^{s-\frac{\alpha+1}{2}, 1}+X^{s-\frac{1+\alpha}{8}, \frac{5}{8}}\right) .
$$

For $u_{0} \in H^{s}(\mathbb{R})$ we will construct a solution to (1-3) that belongs to $Y_{T}^{S}$ for some $T=T\left(\left\|u_{0}\right\|_{H^{1-\alpha / 2}}\right)>0$. As in the regular case, by a dilation argument, we may assume that $L_{\alpha+1}$ satisfies (1-6) for $0<\lambda \leq 1$.

Remark 4.1. Except in the case $(s, \alpha)=(0,2)$, we could simply take $Y^{s, \alpha}:=L_{t}^{\infty} H^{s} \cap X^{s-(\alpha+1) / 2,1}$, since $u \in L^{\infty}\left(0, T ; H^{s}\right)$ forces $\partial_{x}\left(u^{2}\right) \in L^{\infty}\left(0, T ; H^{s-(\alpha+1) / 2}\right)$. To this point of view, $(s, \alpha)=(0,2)$ is a limit case since $u \in L^{\infty}\left(0, T ; L^{2}\right)$ only implies $\partial_{x}\left(u^{2}\right) \in L^{\infty}\left(0, T ; H^{-3 / 2-}\right)$. As in [Zhou 1997], to overcome this difficulty we have to evaluate our solution in Bourgain's spaces with different conormal regularities.

Lemma 4.2. Let $0<T<2,1<\alpha \leq 2, s \geq 1-\frac{\alpha}{2}$ and let $u \in L_{T}^{\infty} H^{s}$ be a solution to (1-3) associated with an initial datum $u_{0} \in H^{s}(\mathbb{R})$. Then $u$ belongs to $Y_{T}^{s, \alpha}$. Moreover, if $(s, \alpha) \neq(0,2)$,

$$
\|u\|_{Y_{T}^{s, \alpha}} \lesssim\|u\|_{L_{T}^{\infty} H^{s}}\left(1+\|u\|_{L_{T}^{\infty} H^{1-\frac{\alpha}{2}}}\right)
$$

and, if $(s, \alpha)=(0,2)$,

$$
\|u\|_{Y_{T}^{0,2}} \lesssim\|u\|_{L_{T}^{\infty} L_{x}^{2}}\left(1+\|u\|_{L_{T}^{\infty} L_{x}^{2}}^{2}\right)
$$

Proof. As in Lemma 3.1 we will work with the extension $\tilde{u}=\rho_{T} u$ of $u$ (see (3-3)). Recall that $\operatorname{supp} \tilde{u} \subset[-2,2] \times \mathbb{R}$ and that

$$
\|\tilde{u}\|_{L_{t}^{\infty} H^{s}} \lesssim\|u\|_{L_{T}^{\infty} H^{s}} \quad \text { and } \quad\|\tilde{u}\|_{X^{\theta, b}} \lesssim\|u\|_{X_{T}^{\theta, b}}
$$

for any $(\theta, b) \in \mathbb{R} \times]-\infty, 1]$. It thus remains to control the $F_{T}^{s, \alpha, \frac{1}{2}}$-norm of $u$. In the case $(s, \alpha) \neq(0,2)$, we actually simply control the $X_{T}^{s-(\alpha+1) / 2,1}$-norm of $u$. Using the integral formulation (see Remark 1.3), standard linear estimates in Bourgain's spaces, and standard product estimates in Sobolev spaces, we infer that

$$
\begin{aligned}
\|u\|_{X_{T}^{s-\frac{1+\alpha}{2}, 1}} \lesssim\left\|u_{0}\right\|_{H^{s-\frac{1+\alpha}{2}}}+\left\|\partial_{x}\left(u^{2}\right)\right\|_{X_{T}^{s-\frac{1+\alpha}{2}, 0}} & \lesssim\left\|u_{0}\right\|_{H^{s-\frac{1+\alpha}{2}}}+\left\|u^{2}\right\|_{L_{T}^{2} H^{s+\frac{1-\alpha}{2}}} \\
& \lesssim\|u\|_{L_{T}^{\infty} H^{s}}+\|u\|_{L_{T}^{\infty} H^{1-\frac{\alpha}{2}}}\|u\|_{L_{T}^{\infty} H^{s}},
\end{aligned}
$$

since, for $1<\alpha \leq 2$ and $s \geq 1-\frac{\alpha}{2}$ with $(s, \alpha) \neq(0,2)$, we have $s+1-\frac{\alpha}{2}>0$ and $s+1-\frac{\alpha}{2}-\left(s+\frac{1-\alpha}{2}\right)=\frac{1}{2}$.

Let us now tackle the case $(s, \alpha)=(0,2)$. First we notice that, since $L^{1}(\mathbb{R}) \hookrightarrow H^{-1 / 2-}(\mathbb{R})$, we have

$$
\|u\|_{X_{T}^{-\frac{7}{4}, 1}} \lesssim\left\|u_{0}\right\|_{H^{-\frac{7}{4}}}+\left\|u^{2}\right\|_{L_{t}^{2} H^{-\frac{3}{4}}} \lesssim\|u\|_{L_{T}^{\infty} L_{x}^{2}}\left(1+\|u\|_{L_{T}^{\infty} L_{x}^{2}}\right)
$$


To bound the $F^{0,2,1 / 2}$-norm of $u$, we first notice that linear estimates in Bourgain's spaces lead to

and then decompose $u^{2}$ as

$$
\|u\|_{F_{T}^{0,2, \frac{1}{2}}} \lesssim\left\|u_{0}\right\|_{H^{-\frac{3}{2}}}+\left\|u^{2}\right\|_{F_{T}^{0,2,-\frac{1}{2}}}
$$

$$
u^{2}=P_{\lesssim 1} u^{2}+\sum_{N \gg 1}\left(P_{N}\left(P_{\ll N} u u_{\sim N}\right)+\sum_{N_{1}^{\prime} \sim N_{1} \gtrsim N} P_{N}\left(u_{N_{1}} u_{N_{1}^{\prime}}\right)\right) .
$$

The contribution of the first term in the right-hand side is easily controlled by $\|u\|_{L_{T}^{\infty} L_{x}^{2}}^{2}$. The contribution of the second term is easily estimated by

$$
\begin{aligned}
\left\|\sum_{N \gg 1} \partial_{x} P_{N}\left(P_{\ll N} u u \sim N\right)\right\|_{F_{T}^{0,2,-\frac{1}{2}}} & \lesssim\left\|\sum_{N \gg 1} P_{N} \partial_{x}\left(P_{\ll N} u u_{\sim N}\right)\right\|_{X_{T}^{-\frac{3}{2}, 0}} \\
& \lesssim\left(\sum_{N \gg 1}\left\|P_{N}\left(P_{\ll N} u u \sim N\right)\right\|_{L_{T}^{2} L_{x}^{1}}^{2}\right)^{\frac{1}{2}} \\
& \lesssim\left(\sum_{N \geq 1}\left\|u_{N}\right\|_{L_{T}^{2} L_{x}^{2}}^{2}\left\|P_{\ll N} u\right\|_{L_{T}^{\infty} L_{x}^{2}}^{2}\right)^{\frac{1}{2}} \\
& \lesssim\|u\|_{L_{T}^{\infty} L^{2}}\|u\|_{L_{T}^{\infty} L_{x}^{2} .}
\end{aligned}
$$

To estimate the third term, we take advantage of the $X^{-3 / 8,-3 / 8}$-part of $F^{0,2,-1 / 2}$. For $N \gg 1$, we have

$$
\begin{aligned}
\sum_{N_{1}^{\prime} \sim N_{1} \gtrsim N}\left\|\partial_{x} P_{N}\left(P_{N_{1}} u P_{N_{1}^{\prime}} u\right)\right\|_{F_{T}^{0,2,-\frac{1}{2}}} \\
\lesssim \sum_{N_{1}^{\prime} \sim N_{1} \gtrsim N} N\left\|\sum_{\substack{\left(L, L_{1}, L_{2}\right) \\
\text { satisfying (2-6) }}} \partial_{x} P_{N} Q_{L}\left(Q_{L_{1}} \tilde{u}_{N_{1}} Q_{L_{2}} \tilde{u}_{N_{1}^{\prime}}\right)\right\|_{X^{-\frac{3}{8},-\frac{3}{8}}} .
\end{aligned}
$$

For the contribution of the sum over $L \gtrsim N N_{1}^{2}$ in (4-7), we obtain

$$
\begin{aligned}
\sum_{N_{1} \sim N_{1}^{\prime} \gtrsim N}\left\|\partial_{x} P_{N} Q_{\gtrsim N N_{1}^{2}}\left(\tilde{u}_{N_{1}} \tilde{u}_{N_{1}^{\prime}}\right)\right\|_{X^{-\frac{3}{8},-\frac{3}{8}}} & \lesssim \sum_{N_{1} \sim N_{1}^{\prime} \gtrsim N} N^{\frac{5}{8}} N^{\frac{1}{2}}\left(N N_{1}^{2}\right)^{-\frac{3}{8}}\left\|\tilde{u}_{N_{1}}\right\|_{L_{t x}^{2}}\left\|\tilde{u}_{N_{1}^{\prime}}\right\|_{L_{t}^{\infty} L_{x}^{2}} \\
& \lesssim\|\tilde{u}\|_{L_{t}^{\infty} L_{x}^{2}} \sum_{N_{1} \gtrsim N}\left(\frac{N}{N_{1}}\right)^{\frac{3}{4}}\left\|\tilde{u}_{N_{1}}\right\|_{L_{t x}^{2}} \\
& \lesssim \gamma_{N}\|\tilde{u}\|_{L_{t}^{\infty} L_{x}^{2}}^{(4-8)}
\end{aligned}
$$

with $\left\|\left(\gamma_{2^{j}}\right)\right\|_{l^{2}(\mathbb{N})} \leq 1$. The contribution of the region where $L \ll N N_{1}^{2}$ and $L_{1} \gtrsim N N_{1}^{2}$ in (4-7) is controlled by

$$
\begin{aligned}
& \sum_{N_{1} \sim N_{1}^{\prime} \approx N}\left\|\partial_{x} P_{N} Q_{\ll N N_{1}^{2}}\left(Q_{\gtrsim N N_{1}^{2}} \tilde{u}_{N_{1}} \tilde{u}_{N_{1}^{\prime}}\right)\right\|_{X^{-\frac{3}{8},-\frac{3}{8}}} \\
& \quad \lesssim \sum_{N_{1} \sim N_{1}^{\prime} \gtrsim N} N^{\frac{5}{8}} N^{\frac{1}{2}}\left(N N_{1}^{2}\right)^{-1} N_{1}^{\frac{7}{4}}\left\|\tilde{u}_{N_{1}}\right\|_{X^{-\frac{7}{4}, 1}}\left\|\tilde{u}_{N_{1}^{\prime}}\right\|_{L_{t}^{\infty} L_{x}^{2}} \lesssim N^{-\frac{1}{8}}\|\tilde{u}\|_{L_{t}^{\infty} L_{x}^{2}}\|\tilde{u}\|_{X^{-\frac{7}{4}, 1}} .
\end{aligned}
$$


Finally, the contribution of the last region, where $L, L_{1} \ll N N_{1}^{2}$ and $L_{2} \sim N N_{1}^{2}$, in (4-7) is controlled in the same way. Gathering (4-4) and (4-7)-(4-9), we obtain the desired result for the case $(s, \alpha)=(0,2)$.

In the sequel we will need the following straightforward estimates.

Lemma 4.3. Let $\alpha \geq 0$ and $w \in F^{0,1 / 2}$. For $1 \leq B \lesssim N^{\alpha+1}$, we have

$$
\left\|Q_{\gtrsim B} w_{N}\right\|_{L^{2}} \lesssim B^{-1} N^{\frac{1+\alpha}{2}}\left\|Q_{\gtrsim B} w_{N}\right\|_{F^{0, \frac{1}{2}}}
$$

and, for $B \gtrsim\langle N\rangle^{\alpha+1}$, we have

$$
\left\|Q_{\gtrsim B} w_{N}\right\|_{L^{2}} \lesssim B^{-\frac{5}{8}}\langle N\rangle^{\frac{1+\alpha}{8}}\left\|Q_{\gtrsim B} w_{N}\right\|_{F^{0, \frac{1}{2}}} .
$$

Proof. Noticing that $F^{0,1 / 2}=F^{0, \alpha, 1 / 2}=X^{-(1+\alpha) / 2,1}+X^{-(1+\alpha) / 8,5 / 8}$, it is easy to check that

$$
\begin{aligned}
\left\|Q_{\gtrsim B} w_{N}\right\|_{L^{2}} & \lesssim \max \left(B^{-1}\langle N\rangle^{\frac{1+\alpha}{2}}, B^{-\frac{5}{8}}\langle N\rangle^{\frac{1+\alpha}{8}}\right)\left\|Q_{\gtrsim B} w_{N}\right\|_{F^{0, \frac{1}{2}}} \\
& \lesssim B^{-\frac{5}{8}}\langle N\rangle^{\frac{1+\alpha}{8}} \max \left(\left(\frac{\langle N\rangle^{1+\alpha}}{B}\right)^{\frac{3}{8}}, 1\right)\left\|Q_{\gtrsim B} w_{N}\right\|_{F^{0, \frac{1}{2}}},
\end{aligned}
$$

which leads to the desired result.

Now we rewrite Lemma 3.2 in the context of the $F^{s, b}$ spaces.

Lemma 4.4. Assume $u_{i} \in Y^{0}, i=1,2,3$, are functions with spatial Fourier support in $\left\{|\xi| \sim N_{i}\right\}$ with $N_{i}>0$ dyadic satisfying $N_{1} \leq N_{2} \leq N_{3}$.

If $N_{3} \gg 1$ and $N_{1} \gtrsim N_{3}^{2(1-\alpha) / 3}$, for $(p, q) \in\{(2, \infty),(\infty, 2)\}$,

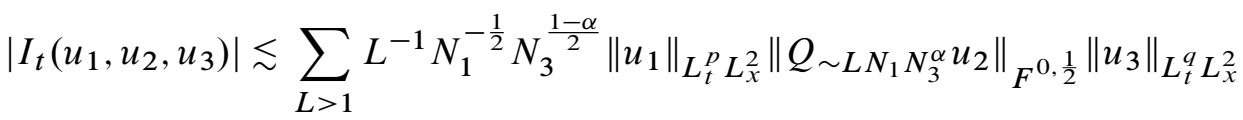

$$
\begin{aligned}
& +N_{1}^{-\frac{1}{2}} N_{3}^{\frac{1-\alpha}{2}}\left\|u_{1}\right\|_{L_{t}^{p} L_{x}^{2}}\left\|u_{2}\right\|_{L_{t}^{q} L_{x}^{2}}\left\|Q_{\sim N_{1} N_{3}^{\alpha}} u_{3}\right\|_{F^{0, \frac{1}{2}}} \\
& +N_{1}^{-\frac{1}{8}}\left\langle N_{1}\right\rangle^{\frac{1+\alpha}{8}} N_{3}^{\frac{-5 \alpha}{8}}\left\|u_{1}\right\|_{F^{0, \frac{1}{2}}}\left\|u_{2}\right\|_{L_{t x}^{2}}\left\|u_{3}\right\|_{L_{t}^{\infty} L_{x}^{2}} \\
& +N_{1}^{-\frac{1}{4}} N_{3}^{\frac{1}{8}-\frac{\alpha}{2}}\left\|u_{1}\right\|_{L_{t}^{\infty} L_{x}^{2}}\left\|u_{2}\right\|_{L_{t}^{\infty} L_{x}^{2}}\left\|u_{3}\right\|_{L_{t}^{\infty} L_{x}^{2}} .
\end{aligned}
$$

Proof. For $R=N_{1}^{3 / 4} N_{3}^{\alpha / 2-1 / 8}$, we decompose $I_{t}$ as in (3-5) and obtain from (3-6) that

$$
\left|I_{t}^{\mathrm{high}}\right| \lesssim N_{1}^{-\frac{1}{4}} N_{3}^{\frac{1}{8}-\frac{\alpha}{2}} \prod_{i=1}^{3}\left\|u_{i}\right\|_{L_{t}^{\infty} L_{x}^{2}} .
$$

To evaluate $I_{t}^{\text {low }}$ we use the decomposition (3-7) and notice that

$$
R=N_{1}^{\frac{3}{4}} N_{3}^{\frac{\alpha}{2}-\frac{1}{8}} \leq N_{1} N_{3}^{\frac{2 \alpha}{3}-\frac{7}{24}} \ll N_{1} N_{3}^{\alpha} \quad \text { and } \quad N_{1} N_{3}^{\alpha} \gtrsim N_{3}^{\frac{2+\alpha}{3}} \gg 1 .
$$

Therefore, the contribution $I_{t}^{1, \text { low }}$ of the first term of the right-hand side of (3-7) to $I_{t}^{\text {low }}$ is easily estimated, thanks to Lemmas 2.5 and 4.3 , by

$$
\left|I_{t}^{1, \text { low }}\right| \lesssim N_{1}^{\frac{1}{2}}\left(N_{1} N_{3}^{\alpha}\right)^{-\frac{5}{8}}\left\langle N_{1}\right\rangle^{\frac{\alpha+1}{8}}\left\|u_{1}\right\|_{F^{0, \frac{1}{2}}}\left\|u_{2}\right\|_{L_{t x}^{2}}\left\|u_{3}\right\|_{L_{t}^{\infty} L_{x}^{2}}
$$


which is acceptable. Thanks to Lemmas $2.3,2.5$ and 4.3 , the contribution $I_{t}^{2 \text {,low }}$ of the second term can be handled in the following way:

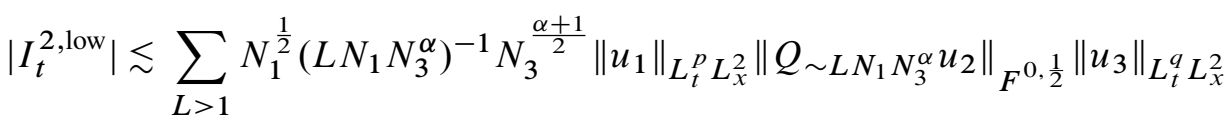

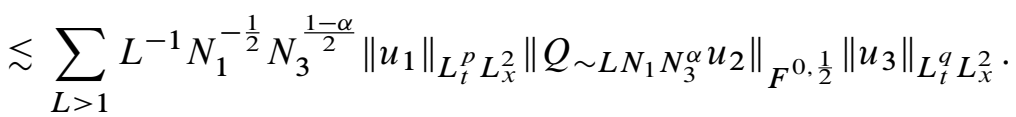

In the same way, we get that the contribution $I_{t}^{3, \text { low }}$ of the third term in $I_{t}^{\text {low }}$ is bounded by

$$
\begin{aligned}
& \left|I_{t}^{3, \text { low }}\right| \lesssim N_{1}^{\frac{1}{2}}\left(N_{1} N_{3}^{\alpha}\right)^{-1} N_{3}^{\frac{\alpha+1}{2}}\left\|u_{1}\right\|_{L_{t}^{p} L_{x}^{2}}\left\|u_{2}\right\|_{L_{t}^{q} L_{x}^{2}} \| Q_{\sim N_{1} N_{3}^{\alpha} u_{3} \|_{F^{0, \frac{1}{2}}}} \\
& \lesssim N_{1}^{-\frac{1}{2}} N_{3}^{\frac{1-\alpha}{2}}\left\|u_{1}\right\|_{L_{t}^{p} L_{x}^{2}}\left\|u_{2}\right\|_{L_{t}^{q} L_{x}^{2}} \| Q_{\sim N_{1} N_{3}^{\alpha} u_{3} \|_{F^{0, \frac{1}{2}}}}
\end{aligned}
$$

Gathering all these estimates, we obtain the desired bound.

Proposition 4.5. Let $0<T<2,1<\alpha \leq 2, s \geq 1-\frac{\alpha}{2}$ and let $u \in L_{T}^{\infty} H^{s}$ be a solution to (1-3) associated with an initial datum $u_{0} \in H^{s}(\mathbb{R})$. Then $u$ belongs to $\tilde{L}_{T}^{\infty} H^{s}$ and

$$
\|u\|_{\widetilde{L}_{T}^{\infty} H^{s}}^{2} \lesssim\left\|u_{0}\right\|_{H^{s}}^{2}+\|u\|_{L_{T}^{\infty} H^{s}}\left(\|u\|_{L_{T}^{\infty} H^{1-\frac{\alpha}{2}}}\|u\|_{Y_{T}^{s}}+\|u\|_{L_{T}^{\infty} H^{s}}\|u\|_{Y_{T}^{1-\frac{\alpha}{2}}}\right) .
$$

Proof. First, we notice that Lemma 4.2 ensures that $u \in Y_{T}^{s}$. Applying the operator $P_{N}$ with $N>0$ dyadic to (1-3), arguing as in (3-11), we obtain

$$
\left\|P_{N} u\right\|_{L_{T}^{\infty} H^{s}}^{2} \lesssim\left\|P_{N} u_{0}\right\|_{H^{s}}^{2}+\sup _{t \in] 0, T[}\langle N\rangle^{2 s}\left|\int_{0}^{t} \int_{\mathbb{R}} P_{N}\left(u^{2}\right) \partial_{x} P_{N} u\right| .
$$

We take an extension $\tilde{u}$ of $u$ supported in time in $]-4,4\left[\right.$ such that $\|\tilde{u}\|_{Y^{s}} \lesssim\|u\|_{Y_{T}^{s}}$. To simplify the notation we drop the tilde in the sequel. We infer from (3-18) that it suffices to estimate

$$
I=\sum_{N>0} \sum_{N_{1} \gtrsim N} N\left\langle N_{1}\right\rangle^{2 s} \sup _{t \in] 0, T[}\left|I_{t}\left(u_{N}, u_{\sim N_{1}}, u_{N_{1}}\right)\right| .
$$

The low frequencies part, $N \lesssim 1$, is estimated exactly as in (3-19) by

$$
\|u\|_{L_{t}^{\infty} L_{x}^{2}}\|u\|_{L_{t}^{\infty} H^{s}}^{2} .
$$

On the other hand, the contribution of the sum over $N \gg 1$ is controlled, thanks to Lemma 4.4 , by

$$
\begin{aligned}
& \sum_{N \gg 1} \sum_{N_{1} \gtrsim N}\left[\left(\frac{N}{N_{1}}\right)^{\frac{\alpha-1}{2}}\left\|u_{N}\right\|_{L_{t}^{2} H^{1-\frac{\alpha}{2}}}\left\|u_{N_{1}}\right\|_{L_{t}^{\infty} H^{s}}\left\|u_{N_{1}}\right\|_{F^{s, \frac{1}{2}}}\right. \\
& +\left(\frac{N}{N_{1}}\right)^{\frac{5 \alpha}{8}}\left\|u_{N}\right\|_{F^{1-\frac{\alpha}{2}, \frac{1}{2}}}\left\|u_{N_{1}}\right\|_{L_{t}^{2} H^{s}}\left\|u_{N_{1}}\right\|_{L_{t}^{\infty} H^{s}} \\
& \left.+N^{\frac{\alpha}{2}-\frac{1}{4}} N_{1}^{\frac{1}{8}-\frac{\alpha}{2}}\left\|u_{N}\right\|_{L_{t}^{\infty} H^{1-\frac{\alpha}{2}}}\left\|u_{N_{1}}\right\|_{L_{t}^{\infty} H^{s}}^{2}\right] \\
& \lesssim\|u\|_{Y^{1-\frac{\alpha}{2}}}\|u\|_{L_{t}^{\infty} H^{s}}^{2}+\|u\|_{L_{t}^{\infty} H^{1-\frac{\alpha}{2}}}\|u\|_{L_{t}^{\infty} H^{s}}\|u\|_{Y^{s}},
\end{aligned}
$$


where we use the discrete Young's inequality in $N_{1}$ and then Cauchy-Schwarz in $N$ to bound the first two terms.

Gathering the above estimates we eventually obtain

$$
I \lesssim\|u\|_{Y_{T}^{1-\frac{\alpha}{2}}}\|u\|_{L_{T}^{\infty} H^{s}}^{2}+\|u\|_{L_{T}^{\infty} H^{1-\frac{\alpha}{2}}}\|u\|_{L_{T}^{\infty} H^{s}}\|u\|_{Y_{T}^{s}}
$$

which completes the proof of the proposition.

4A. Estimates on the difference of two solutions. First we introduce the function spaces where we will estimate the difference of two solutions of (1-3). Contrary to the regular case, we will have to work in a function space that puts a weight on the very low frequencies. This kind of weighted space for the difference of two solutions was, for instance, used in [Ionescu et al. 2008] in the context of short-time Bourgain spaces.

For $\theta \in \mathbb{R}$ we define the Banach space

$$
\bar{H}^{\theta}(\mathbb{R})=\left\{\varphi \in H^{\theta}(\mathbb{R}) \mid\|\varphi\|_{\bar{H}^{\theta}}<\infty\right\}
$$

with

$$
\|\varphi\|_{\bar{H}^{\theta}}:=\left\|\left\langle|\xi|^{-\frac{1}{2}}\right\rangle\langle\xi\rangle^{\theta} \hat{\varphi}\right\|_{L^{2}}
$$

equipped with the norm $\|\cdot\|_{\bar{H}^{\theta}}$. Then we define the space $\widetilde{L}_{t}^{\infty} \bar{H}^{\theta}$ by

$$
\|w\|_{\tilde{L}_{t}^{\infty} \bar{H}^{\theta}}:=\left(\sum_{N>0}\left\|w_{N}\right\|_{L_{t}^{\infty} \bar{H}^{\theta}}^{2}\right)^{\frac{1}{2}} .
$$

Finally, we define the function spaces $\tilde{Y}^{\theta}$ and $Z^{\theta}, \theta \in \mathbb{R}$, by

$$
\tilde{Y}^{\theta}=\tilde{L}_{t}^{\infty} H^{\theta} \cap F^{\theta, \frac{1}{2}} \quad \text { and } \quad Z^{\theta}=\tilde{L}_{t}^{\infty} \bar{H}^{\theta} \cap F^{\theta, \frac{1}{2}}
$$

with $F^{\theta, b}$ as defined in (4-1).

If $u, v \in L_{T}^{\infty} H^{s}$ are two solutions of (1-3) with $s \geq 1-\frac{\alpha}{2}$, then, by Lemma 4.2 and Proposition 4.5, we know that $u$ and $v$ belong to $Y_{T}^{S} \cap \widetilde{L}_{T}^{\infty} H^{s}$. Moreover, again using the extension operator $\rho_{T}$, it is easy to check that

$$
Y_{T}^{s} \cap \widetilde{L}_{T}^{\infty} H^{s} \hookrightarrow \tilde{Y}_{T}^{s}
$$

with an embedding constant that does not depend on $0<T \leq 2$. Hence, $u$ and $v$ belong to $\tilde{Y}_{T}^{s}$. Assuming that $u_{0}-v_{0} \in \bar{H}^{s}$, we claim that the difference $u-v$ belongs to $Z_{T}^{s}$. Indeed, according to the above definitions of $\widetilde{Y}^{s}$ and $Z^{s}$, it suffices to check that $P_{1}(u-v)$ belongs to $\widetilde{L}_{T}^{\infty} \bar{H}^{s}$. But this is straightforward, since, by the Duhamel formula, for any dyadic integer $0<N<1$ we have

$$
\left\|P_{N}(u-v)\right\|_{L_{T}^{\infty} \bar{H}^{s}} \lesssim\left\|u_{0}-v_{0}\right\|_{\bar{H}^{s}}+N^{\frac{1}{2}}\left(\|u\|_{L_{T}^{\infty} L_{x}^{2}}^{2}+\|v\|_{L_{T}^{\infty} L_{x}^{2}}^{2}\right)
$$

We are thus allowed to estimate the difference $w=u-v$ in the space $Z_{T}^{s-3 / 2+\alpha / 2}$. 
Remark 4.6. For $\alpha>1$, we have $s-\frac{3}{2}+\frac{\alpha}{2}>s-1$ and thus, contrary to the preceding section, the derivative of a solution does not belong to the space where we estimate the difference $w=u-v$ of two solutions. This fact is crucial in the preceding section to recover the derivative in terms as $J_{2}$ in (3-27) that contains small space frequencies of $w$. In this section, we will instead combine the weight on the low space frequencies of $w$ with the resonance relation to control such contributions.

Proposition 4.7. Let $0<T<2,1<\alpha \leq 2, s \geq 1-\frac{\alpha}{2}$ and $u, v \in L_{T}^{\infty} H^{s}$ be two solutions to (1-3) on ] $0, T\left[\right.$ associated with initial data $u_{0}, v_{0} \in H^{s}$ such that $u_{0}-v_{0} \in \bar{H}^{s}$. Then $u-v \in Z_{T}^{s-3 / 2+\alpha / 2}$ and we have

$$
\|u-v\|_{Z_{T}^{s-\frac{3}{2}+\frac{\alpha}{2}}} \lesssim\|u-v\|_{L_{T}^{\infty} \bar{H}^{s-\frac{3}{2}+\frac{\alpha}{2}}}+\|u+v\|_{\tilde{Y}_{T}^{s}}\|u-v\|_{Z_{T}^{-\frac{1}{2}}}+\|u+v\|_{\widetilde{Y}^{1-\frac{\alpha}{2}}}\|u-v\|_{Z_{T}^{s-\frac{3}{2}+\frac{\alpha}{2}}} \cdot
$$

Proof. The fact that $u-v \in Z_{T}^{s-3 / 2+\alpha / 2}$ follows from the discussion above. Now, recall that $w=u-v$ satisfies (3-23) with $z=u+v$. We extend $w$ from $(0, T)$ to $\mathbb{R}$ by using the extension operator $\rho_{T}$ defined in (3-3). On account of the uniform bounds on $\rho_{T}$ (see the paragraph just after (3-3)), it remains to estimate the $F_{T}^{s-3 / 2+\alpha / 2, \alpha, 1 / 2}$-norm of $w$. From classical linear estimates in the framework of Bourgain's spaces, the Duhamel formulation associated with (3-23) leads to

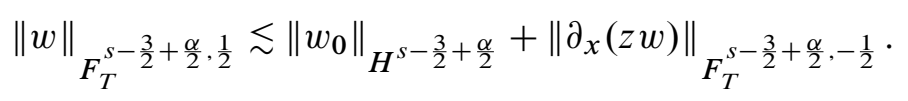

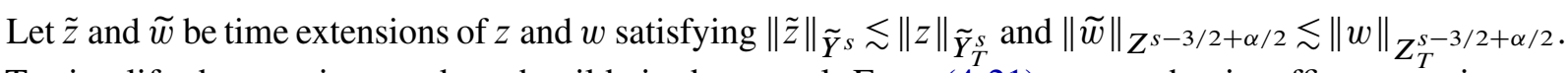
To simplify the notation we drop the tilde in the sequel. From (4-21) we see that it suffices to estimate

$$
\left\|\partial_{x}(z w)\right\|_{F^{s-\frac{3}{2}+\frac{\alpha}{2},-\frac{1}{2}}} \lesssim\left(\sum_{N>0}\left\|P_{N} \partial_{x}(z w)\right\|_{F^{s-\frac{3}{2}+\frac{\alpha}{2},-\frac{1}{2}}}^{2}\right)^{\frac{1}{2}} .
$$

We first estimate the low-high contribution $P_{N}\left(P_{\lesssim N} z P_{\sim N} w\right)$ :

$$
\begin{aligned}
& \left\|\partial_{x} P_{N}\left(P_{\lesssim N} z P \sim N w\right)\right\|_{F^{s-\frac{3}{2}+\frac{\alpha}{2},-\frac{1}{2}}} \lesssim \sum_{N_{1} \lesssim N} N\left\|P_{N}\left(P_{N_{1}} z P \sim N w\right)\right\|_{X^{s-2,0}} \\
& \lesssim \sum_{N_{1} \lesssim N} N_{1}^{\frac{1}{2}} N\langle N\rangle^{s-2}\left\|P_{N_{1}} z\right\|_{L_{t}^{\infty} L_{x}^{2}}\left\|P_{\sim N} w\right\|_{L_{t}^{2} L_{x}^{2}} \\
& \lesssim\left\|P_{\sim N} w\right\|_{L_{t}^{2} H^{s-\frac{3}{2}+\frac{\alpha}{2}}} \sum_{N_{1} \lesssim N}\left(\frac{N_{1}}{\langle N\rangle}\right)^{\frac{\alpha-1}{2}}\left\|P_{N_{1}} z\right\|_{L_{t}^{\infty} H^{1-\frac{\alpha}{2}}} \\
& \lesssim\|z\|_{L_{t}^{\infty} H^{1-\frac{\alpha}{2}}}\left\|P_{\sim N} w\right\|_{L_{t}^{\infty} H^{s-\frac{3}{2}+\frac{\alpha}{2}}}
\end{aligned}
$$

Similarly, the high-low interactions are estimated as follows:

$$
\begin{aligned}
\left\|\partial_{x} P_{N}\left(P_{\sim N} z P_{\lesssim N} w\right)\right\|_{F^{s-\frac{3}{2}+\frac{\alpha}{2},-\frac{1}{2}}} & \lesssim N\left\|P_{N}\left(P_{\sim N} z P_{\lesssim N} w\right)\right\|_{X^{s-2,0}} \\
& \lesssim\left\|P_{\sim N} z\right\|_{L_{t}^{2} H^{s}} \sum_{N_{1} \lesssim N}\left(\frac{N_{1}}{\langle N\rangle}\right)^{\frac{1}{2}}\left\|P_{N_{1}} w\right\|_{L_{t}^{\infty} H^{-\frac{1}{2}}} \\
& \lesssim\left\|P_{\sim N} z\right\|_{L_{t}^{2} H^{s}}\|w\|_{L_{t}^{\infty} H^{-\frac{1}{2}}} \cdot
\end{aligned}
$$


Now we deal with the high-high interactions term:

$$
\left\|\partial_{x} P_{N}\left(P_{\gg N} z P_{\gg N} w\right)\right\|_{F^{s-\frac{3}{2}+\frac{\alpha}{2},-\frac{1}{2}} \lesssim} \sum_{N_{1} \gg N} N\left\|\sum_{\substack{\left(L, L_{1}, L_{2}\right) \\ \text { satisfying }(2-6)}} \partial_{x} P_{N} Q_{L}\left(Q_{L_{1}} z_{N_{1}} Q_{L_{2}} w_{N_{1}}\right)\right\|_{F^{s-\frac{3}{2}+\frac{\alpha}{2},-\frac{1}{2}}} .
$$

We may assume that $N_{1} \gg 1$ since, otherwise, $N \ll N_{1} \lesssim 1$ and we have

$$
\left\|P_{\lesssim 1} \partial_{x}\left(P_{\lesssim 1} z P_{\lesssim 1} w\right)\right\|_{F^{s-\frac{3}{2}}+\frac{\alpha}{2},-\frac{1}{2}} \lesssim\left\|P_{\lesssim 1} z\right\|_{L_{t}^{\infty} L^{2}}\left\|P_{\lesssim 1} w\right\|_{L_{t}^{\infty} H^{-\frac{1}{2}}}
$$

For $N_{1} \gg 1$, we will take advantage of the fact that $X^{s-13 / 8+3 \alpha / 8,-3 / 8} \hookrightarrow F^{s-3 / 2+\alpha / 2,-1 / 2}$. The contribution of the sum over $L \gtrsim N N_{1}^{\alpha}$ can be thus controlled by

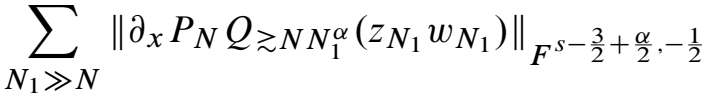

$$
\begin{aligned}
& \lesssim \sum_{N_{1} \gg N} N\left\|P_{N} Q_{\gtrsim N N_{1}^{\alpha}}\left(z_{N_{1}} w_{N_{1}}\right)\right\|_{X^{s-\frac{13}{8}}+\frac{3 \alpha}{8},-\frac{3}{8}} \\
& \lesssim \sum_{N_{1} \gg N} \sum_{L \gtrsim N N_{1}^{\alpha}} N\langle N\rangle^{s-\frac{13}{8}+\frac{3 \alpha}{8}} L^{-\frac{3}{8}}\left\|P_{N} Q_{L}\left(z_{N_{1}} w_{N_{1}}\right)\right\|_{L^{2}} \\
& \lesssim \sum_{N_{1} \gg N} N^{\frac{3}{2}}\langle N\rangle^{s-\frac{13}{8}+\frac{3 \alpha}{8}}\left(N N_{1}^{\alpha}\right)^{-\frac{3}{8}} N_{1}^{\frac{1}{2}-s}\left\|z_{N_{1}}\right\|_{L_{t}^{2} H^{s}}\left\|w_{N_{1}}\right\|_{L_{t}^{\infty} H^{-\frac{1}{2}}} \\
& \lesssim \sum_{N_{1} \gg N}\left(\frac{N}{N_{1}}\right)^{\frac{1}{2}-\frac{\alpha}{8}}\left(\frac{\langle N\rangle}{\left\langle N_{1}\right\rangle}\right)^{s-1+\frac{\alpha}{2}}\left\|z_{N_{1}}\right\|_{L_{t}^{2} H^{s}}\left\|w_{N_{1}}\right\|_{L_{t}^{\infty} H^{-\frac{1}{2}}} \\
& \lesssim \delta_{N}\|z\|_{L_{t}^{2} H^{s}}\|w\|_{L_{t}^{\infty} H^{-\frac{1}{2}}},
\end{aligned}
$$

where $\left\|\left(\delta_{2^{j}}\right)_{j}\right\|_{l^{2}(\mathbb{Z})} \lesssim 1$. The contribution of the region where $L \ll N N_{1}^{\alpha}$ and $L_{1} \gtrsim N N_{1}^{\alpha}$ is estimated, thanks to (4-10), by

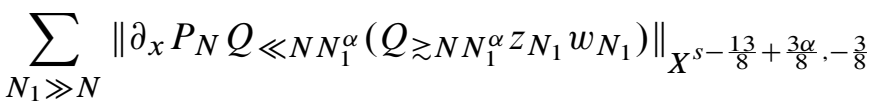

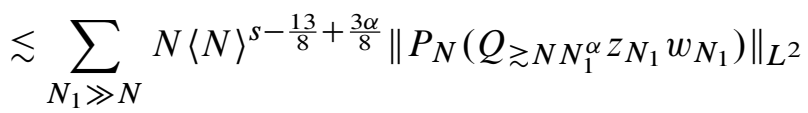

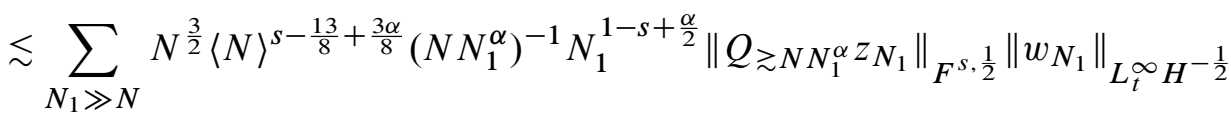

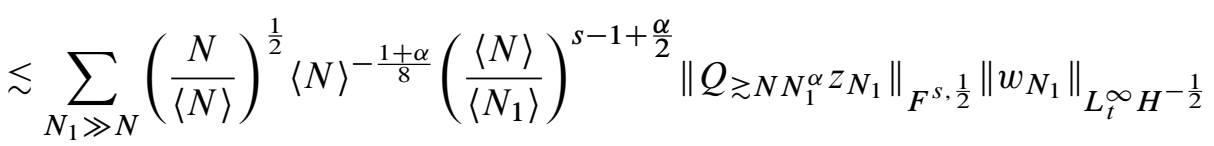

$$
\begin{aligned}
& \lesssim \delta_{N}\|z\|_{Y^{s}}\|w\|_{\widetilde{L}_{t}^{\infty} H^{-\frac{1}{2}}},
\end{aligned}
$$


where $\left\|\left(\delta_{2^{j}}\right)_{j}\right\|_{l^{2}(\mathbb{Z})} \lesssim 1$. Finally the contribution of the last region can be bounded, thanks to (4-10), by

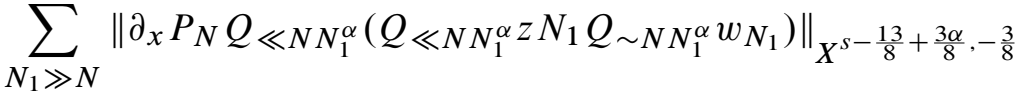

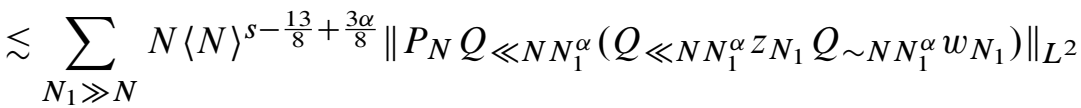

$$
\begin{aligned}
& \lesssim \sum_{N_{1} \gg N} N^{\frac{3}{2}}\langle N\rangle^{s-\frac{13}{8}+\frac{3 \alpha}{8}} N_{1}^{-s}\left(N N_{1}^{\alpha}\right)^{-1} N_{1}^{1+\frac{\alpha}{2}}\left\|Q_{\ll N N_{1}^{\alpha} z_{N_{1}}}\right\|_{L_{t}^{\infty} H^{s}}\left\|Q_{\sim N N_{1}^{\alpha}} w_{N_{1}}\right\|_{F^{-\frac{1}{2}}, \frac{1}{2}} \\
& \lesssim \sum_{N_{1} \gg N}\left(\frac{N}{\langle N\rangle}\right)^{\frac{1}{2}}\langle N\rangle^{-\frac{1+\alpha}{8}}\left(\frac{\langle N\rangle}{\left\langle N_{1}\right\rangle}\right)^{s-1+\frac{\alpha}{2}}\left\|z_{N_{1}}\right\|_{L_{t}^{\infty} H^{s}}\left\|w_{N_{1}}\right\|_{F^{-\frac{1}{2}, \frac{1}{2}}} \\
& \lesssim \delta_{N}\|z\|_{\tilde{L}_{t}^{\infty} H^{s}}\|w\|_{Z^{-\frac{1}{2}}},
\end{aligned}
$$

which is acceptable. This concludes the proof of Proposition 4.7.

Proposition 4.8. Let $1 \leq \alpha \leq 2,0<T<2$ and let $u, v \in L_{T}^{\infty} H^{s}$ with $s \geq 1-\frac{\alpha}{2}$ be two solutions to (1-3) associated with initial data $u_{0}, v_{0} \in H^{s}$ such that $u_{0}-v_{0} \in \bar{H}^{s}$. Then ${ }^{1}$

$$
\|u-v\|_{\tilde{L}_{T}^{\infty} \bar{H}^{s-\frac{3}{2}+\frac{\alpha}{2}}}^{2} \lesssim\left\|u_{0}-v_{0}\right\|_{\bar{H}^{s-\frac{3}{2}+\frac{\alpha}{2}}}^{2}+\|u+v\|_{Y_{T}^{s}}\|u-v\|_{\tilde{L}_{T}^{\infty} \bar{H}^{s-\frac{3}{2}+\frac{\alpha}{2}}}\|u-v\|_{Z_{T}^{s-\frac{3}{2}+\frac{\alpha}{2}}} \cdot
$$

Proof. Recall that the difference $w=u-v$ satisfies (3-23) with $z=u+v$. Applying the operator $P_{N}$ with $N>0$ dyadic to (3-23), taking the $L^{2}$ scalar product with $P_{N} w$ and integrating on ]0,t [, we obtain

$$
\left\|w_{N}\right\|_{L_{T}^{\infty} \bar{H}^{s-\frac{3}{2}+\frac{\alpha}{2}}}^{2} \lesssim\left\|P_{N} w_{0}\right\|_{\bar{H}^{s-\frac{3}{2}+\frac{\alpha}{2}}}+\left\langle N^{-1}\right\rangle\langle N\rangle^{2\left(s-\frac{3}{2}+\frac{\alpha}{2}\right)} \sup _{t \in[0, T]}\left|\int_{0}^{t} \int_{\mathbb{R}} P_{N}(z w) \partial_{x} w_{N}\right| .
$$

Therefore, we have to estimate

$$
J:=\sum_{N>0}\left\langle N^{-1}\right\rangle\langle N\rangle^{2\left(s-\frac{3-\alpha}{2}\right)} \sup _{t \in[0, T]}\left|\int_{0}^{t} \int_{\mathbb{R}} P_{N}(z w) \partial_{x} w_{N}\right| .
$$

We take extensions $\tilde{z}$ and $\tilde{w}$ of $z$ and $w$ supported in time in ]-4, 4[ such that $\|\tilde{z}\|_{Y^{s}} \lesssim\|u\|_{Y_{T}^{s}}$ and $\|\tilde{w}\|_{Z^{s}} \lesssim\|w\|_{Z_{T}^{s}}$. To simplify the notation we drop the tilde in the sequel.

Proceeding as in (3-27), we get

$$
\begin{aligned}
& J \lesssim \sum_{N>0} \\
& \sum_{N_{1} \gtrsim N} N\left\langle N_{1}^{-1}\right\rangle\left\langle N_{1}\right\rangle^{2\left(s-\frac{3-\alpha}{2}\right)} \sup _{t \in] 0, T[}\left|I_{t}\left(z_{N}, w_{\sim N_{1}}, w_{N_{1}}\right)\right| \\
&+\sum_{N>0} \sum_{N_{1} \gtrsim N} N_{1}\left\langle N_{1}^{-1}\right\rangle\left\langle N_{1}\right\rangle^{2\left(s-\frac{3-\alpha}{2}\right)} \sup _{t \in] 0, T[}\left|I_{t}\left(z_{\sim N_{1}}, w_{N}, w_{N_{1}}\right)\right| \\
& \quad+\sum_{N>0} \sum_{N_{1} \gtrsim N} N\left\langle N^{-1}\right\rangle\langle N\rangle^{2\left(s-\frac{3-\alpha}{2}\right)} \sup _{t \in] 0, T[}\left|I_{t}\left(z_{N_{1}}, w_{N_{1}}, w_{N}\right)\right| \\
&:=J_{1}+J_{2}+J_{3} .
\end{aligned}
$$

\footnotetext{
${ }^{1}$ We include the case $\alpha=1$ here since it does not lead to additional difficulties and will be useful in the Appendix to prove LWP for $(\alpha, s)=\left(1, \frac{1}{2}\right)$.
} 
Estimates for $J_{1}$. The contribution of the sum over $N \lesssim 1$ in $J_{1}$ is estimated, thanks to (3-4), by

$$
\sum_{N \lesssim 1} \sum_{N_{1} \gtrsim N} N^{\frac{3}{2}}\left\|z_{N}\right\|_{L_{t}^{\infty} L_{x}^{2}}\left\|w_{N_{1}}\right\|_{L_{t}^{\infty} \bar{H}^{s-\frac{3-\alpha}{2}}}^{2} \lesssim\|z\|_{L_{t}^{\infty} L_{x}^{2}}\|w\|_{\tilde{L}_{T}^{\infty} \bar{H}^{s-\frac{3-\alpha}{2}}}^{2} .
$$

The contribution $N \gg 1$ in $J_{1}$ can be controlled with Lemma 4.4 by

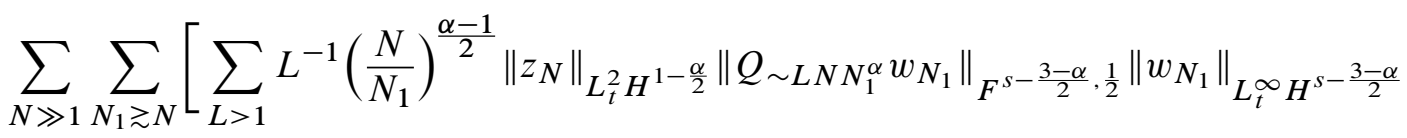

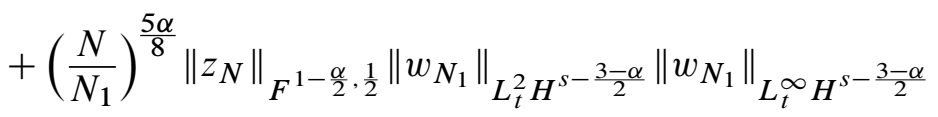

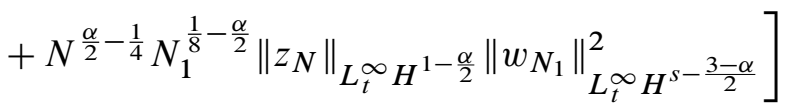

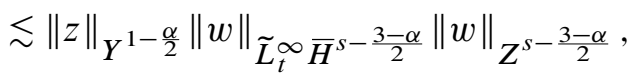

where for the first term we used Cauchy-Schwarz in $\left(N, N_{1}\right)$ and then summed in $L$. Note that for $\alpha>1$ we could replace the $\widetilde{L}_{t}^{\infty} H^{s-3 / 2+\alpha / 2}$-norm by a standard $L_{t}^{\infty} H^{s-3 / 2+\alpha / 2}$-norm by invoking the discrete Young inequality.

Estimates for $J_{2}$. We separate different contributions. First, the contribution of the sum over $N_{1} \lesssim 1$

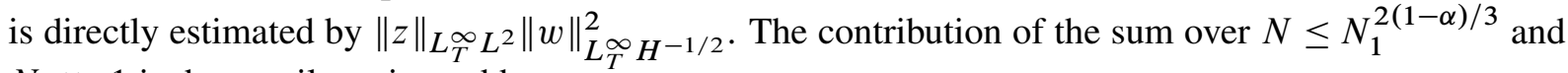
$N_{1} \gg 1$ is then easily estimated by

$$
\begin{aligned}
\sum_{N_{1} \gg 1} \sum_{N \leq N_{1}^{\frac{2}{3}(1-\alpha)}} N N_{1}^{\frac{\alpha-1}{2}}\left\|z_{N_{1}}\right\|_{L_{T}^{2} H^{s}}\left\|w_{N}\right\|_{L_{T}^{\infty} \bar{H}^{-\frac{1}{2}}}\left\|w_{N_{1}}\right\|_{L_{T}^{2} H^{s-\frac{3}{2}+\frac{\alpha}{2}}} & \\
& \lesssim \sum_{N_{1} \gg 1} N_{1}^{\frac{1-\alpha}{6}}\left\|z_{N_{1}}\right\|_{L_{T}^{2} H^{s}}\|w\|_{L_{T}^{\infty} \bar{H}^{-\frac{1}{2}}}\left\|w_{N_{1}}\right\|_{L_{T}^{2} H^{s-\frac{3}{2}+\frac{\alpha}{2}}} \\
& \lesssim\|z\|_{L_{T}^{\infty} H^{s}}\|w\|_{L_{T}^{\infty} \bar{H}^{-\frac{1}{2}}}\|w\|_{L_{T}^{\infty} H^{s-\frac{3}{2}+\frac{\alpha}{2}}}
\end{aligned}
$$

Finally the contribution of the sum over $N_{1} \gg 1$ and $N \gg N_{1}^{2(1-\alpha) / 3}$ is bounded, thanks to Lemma 4.4 , by

$$
\begin{aligned}
& \sum_{N_{1} \gg 1} \sum_{\substack{\frac{2}{3}(1-\alpha) \\
N \gg N_{1}^{3}}}\left[\sum_{L>1}\left\|w_{N}\right\|_{L_{t}^{\infty} \bar{H}^{-\frac{1}{2}}}\left\|Q_{\sim L N N_{1}^{\alpha}} w_{N_{1}}\right\|_{F^{s-\frac{3-\alpha}{2}, \frac{1}{2}}}\left\|z_{N_{1}}\right\|_{L_{t}^{2} H^{s}}\right. \\
& +\left\|w_{N}\right\|_{L_{t}^{\infty} \bar{H}^{-\frac{1}{2}}}\left\|w_{N_{1}}\right\|_{L_{t}^{2} H^{s-\frac{3-\alpha}{2}}}\left\|Q \sim N N_{1}^{\alpha} z_{N_{1}}\right\|_{F^{s, \frac{1}{2}}}
\end{aligned}
$$

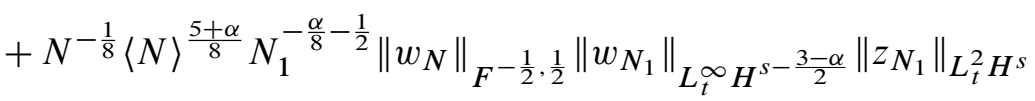

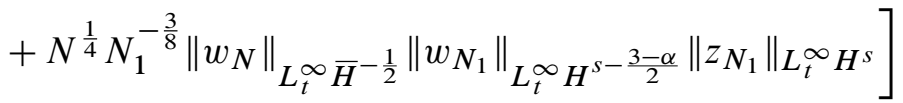

$$
\begin{aligned}
& \lesssim\|z\| Y^{s}\left(\|w\|_{\tilde{L}_{t}^{\infty} \bar{H}^{-\frac{1}{2}}}\|w\|_{Z^{s-\frac{3-\alpha}{2}}}+\|w\|_{Z^{-\frac{1}{2}}}\|w\|_{\tilde{L}_{t}^{\infty} \bar{H}^{-\frac{1}{2}}}\right),
\end{aligned}
$$

where again we used Cauchy-Schwarz in $\left(N, N_{1}\right)$ and then summed over $L$. 
Estimates for $J_{3}$. We first notice that for $N \lesssim N_{1}$ and $N_{1} \gg 1$, since $1+2\left(s-\frac{3-\alpha}{2}\right) \geq 0$,

$$
N\left\langle N^{-1}\right\rangle\langle N\rangle^{2\left(s-\frac{3-\alpha}{2}\right)} \lesssim N_{1}\left\langle N_{1}^{-1}\right\rangle\left\langle N_{1}\right\rangle^{2\left(s-\frac{3-\alpha}{2}\right)} .
$$

Therefore, the contribution of this region to $J_{3}$ is controlled by $J_{2}$. Finally the contribution of $N \lesssim N_{1} \lesssim 1$ is easily bounded by $\|z\|_{L_{t}^{\infty} L_{x}^{2}}\|w\|_{L_{t}^{\infty} \bar{H}^{-1 / 2}}^{2}$.

Gathering all the estimates, we eventually obtain

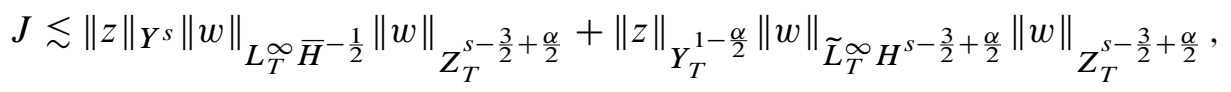

which completes the proof of (4-22).

4B. Unconditional well-posedness. Let us fix $s \geq 1-\frac{\alpha}{2}$. We notice that $1-\frac{\alpha}{2} \geq 0>s_{c}=\frac{1}{2}-\alpha$, which is the critical Sobolev exponent associated with (1-3) for dilation symmetry. Therefore, as in Section 3B, the unconditional well-posedness in $H^{s}(\mathbb{R})$ of (1-3) for small $H^{s}$-initial data with a maximal time of existence $T \geq 1$ will ensure the unconditional well-posedness of (1-3) for arbitrary large $H^{s}$-initial data with a maximal time of existence

$$
T \geq\left(1+\left\|u_{0}\right\|_{H^{s}}\right)^{-\frac{2(\alpha+1)}{2 \alpha-1}} .
$$

Moreover, as in Section 3B, estimates (4-2), (4-3), (4-14), and a continuity argument ensure that a smooth solution with small $H^{s}$-initial datum has got a time of existence $T$ in $H^{\infty}(\mathbb{R})$ that is greater than 1 . Now, to prove the existence of a solution with initial data $u_{0} \in H^{1-\alpha / 2}$, we cannot argue exactly as in Section 3B since, for $s=0$, we miss compactness to pass to the limit on the nonlinear term. Instead, we construct below a sequence of smooth solutions to (1-3) that converges strongly to a solution of (1-3) emanating from $u_{0}$. This will be done by using the Bona-Smith argument.

Let $u_{0} \in H^{s}$ with $s \geq 1-\frac{\alpha}{2}$ and $\left\|u_{0}\right\|_{H^{s}} \ll 1$. We denote by $u^{N}$ the solution of (1-3) emanating from $P_{\leq N} u_{0}$. From the discussion above, $u_{N} \in C\left([0,1] ; H^{\infty}(\mathbb{R})\right)$ and, for $1 \leq N_{1} \leq N_{2}$, we set

$$
w:=u^{N_{1}}-u^{N_{2}} \text {. }
$$

Let us note that $P_{\leq 1} w_{0}=P_{\leq 1}\left(u^{N_{1}}-u^{N_{2}}\right)=0$ and thus $w_{0} \in \bar{H}^{s}(\mathbb{R})$ with $\left\|w_{0}\right\|_{\bar{H}^{s}} \sim\left\|w_{0}\right\|_{H^{s}}$. It then follows from (4-20)-(4-22) that

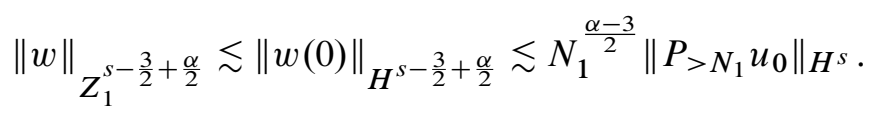

Moreover, on account of Lemma 4.2, Proposition 4.5 and (4-19), for any $r \geq 0$ we have

$$
\left\|u^{N_{i}}\right\|_{Y_{T}^{s+r}} \lesssim\left\|u^{N_{i}}\right\|_{\widetilde{Y}_{T}^{s+r}} \lesssim\left\|u_{0}^{N_{i}}\right\|_{H^{s+r}} \lesssim N_{i}^{r}\left\|u_{0}\right\|_{H^{s}} .
$$

Next, since $w$ satisfies (3-36), the Duhamel formula leads, for any $0<N<1$, to

and thus

$$
\left\|P_{N} w\right\|_{L_{1}^{\infty} \bar{H}^{s}} \lesssim\left\|P_{N} w_{0}\right\|_{\bar{H}^{s}}+N^{\frac{1}{2}}\left(\left\|u^{N_{1}}\right\|_{L_{1}^{\infty} L_{x}^{2}}^{2}+\|w\|_{L_{1}^{\infty} L_{x}^{2}}^{2}\right)
$$

$$
\left\|P_{\leq 1} w\right\|_{\tilde{L}_{1}^{\infty} \bar{H}^{s}} \lesssim\left\|w_{0}\right\|_{H^{s}}+\left(\left\|u^{N_{1}}\right\|_{L_{1}^{\infty} L_{x}^{2}}^{2}+\|w\|_{L_{1}^{\infty} L_{x}^{2}}^{2}\right)
$$


This proves that $w \in Z_{T}^{s}$. We will also need the following estimates on $w$ :

Proposition 4.9. Let $1<\alpha \leq 2,0<T<2$ and $w \in Z_{T}^{s}$ with $s \geq 1-\frac{\alpha}{2}$ be a solution to (3-36). Then

and

$$
\|w\|_{Y_{T}^{s}} \lesssim\|w\|_{L_{T}^{\infty} H^{s}}\left(1+\left\|u^{N_{1}}\right\|_{L_{T}^{\infty} H^{s}}^{2}+\|w\|_{L_{T}^{\infty} H^{s}}^{2}\right)
$$

$$
\|w\|_{\widetilde{L}_{T}^{\infty} H^{s}}^{2} \lesssim\left\|w_{0}\right\|_{H^{s}}^{2}+\|w\|_{Y_{T}^{s}}^{3}+\left\|u^{N_{1}}\right\|_{Y_{T}^{s}}\|w\|_{Z_{T}^{s}}^{2}+\left\|u^{N_{1}}\right\|_{Y_{T}^{s+\frac{3}{2}-\frac{\alpha}{2}}}\|w\|_{Z_{T}^{s-\frac{3}{2}}+\frac{\alpha}{2}}\|w\|_{Z_{T}^{s}} .
$$

Proof. First, (4-29) can be derived exactly as (4-2)-(4-3) of Lemma 4.2. Now, to prove (4-30), we separate the contribution of $\partial_{x}\left(w^{2}\right)$ and $\partial_{x}\left(u^{N_{1}} w\right)$. First, (4-17) leads to

$$
\sum_{N>0} N^{2 s}\left|\int_{0}^{t} \int_{\mathbb{R}} P_{N} \partial_{x}\left(w^{2}\right) P_{N} w\right| \lesssim\|w\|_{Y}^{3}
$$

Second, applying (4-25) at the level $s$ with $z$ replaced by $u^{N_{1}}$, we obtain

$$
\sum_{N>0} N^{2 s}\left|\int_{0}^{t} \int_{\mathbb{R}} P_{N} \partial_{x}\left(u^{N_{1}} w\right) P_{N} w\right| \lesssim\left\|u^{N_{1}}\right\|_{Y_{T}^{s+\frac{3}{2}-\frac{\alpha}{2}}}\|w\|_{Z_{T}^{-\frac{1}{2}}}\|w\|_{Z_{T}^{s}}+\left\|u^{N_{1}}\right\|_{Y_{T}^{1-\frac{\alpha}{2}}}\|w\|_{Z_{T}^{s}}^{2},
$$

which leads to (4-30) since $s-\frac{3}{2}+\frac{\alpha}{2} \geq-\frac{1}{2}$ for $s \geq 1-\frac{\alpha}{2}$ and $Z_{T}^{S} \hookrightarrow Y_{T}^{S}$.

Combining (4-28), (4-29), (4-30) and (4-19), we infer that

$$
\begin{aligned}
& \|w\|_{Z_{1}^{s}}^{2} \\
& \quad \lesssim\left(1+\left\|u_{0}\right\|_{H^{s}}^{2}\right)^{2}\left[\left\|w_{0}\right\|_{H^{s}}^{2}+\left\|u_{0}\right\|_{H^{s}}\|w\|_{Y_{1}^{s}}^{2}+\left\|u_{0}\right\|_{H^{s}}\|w\|_{Z_{1}^{s}}^{2}+N_{1}^{\frac{3-\alpha}{2}}\left\|u_{0}\right\|_{H^{s}}\|w\|_{Z_{1}^{s-\frac{3}{2}}+\frac{\alpha}{2}}\|w\|_{Z_{1}^{s}}\right] .
\end{aligned}
$$

Then, the smallness assumption on $\left\|u_{0}\right\|_{H^{s}},(4-26)$ and the continuous injection $Z_{T}^{s} \hookrightarrow Y_{T}^{s}$, lead to

$$
\begin{aligned}
\|w\|_{Z_{1}^{s}}^{2} & \lesssim\left\|w_{0}\right\|_{H^{s}}^{2}+N_{1}^{3-\alpha}\|w\|_{Z_{1}^{s-\frac{3}{2}+\frac{\alpha}{2}}}^{2} \\
& \lesssim\left\|P_{>N_{1}} u_{0}\right\|_{H^{s}}^{2}\left(1+\left\|P_{>N_{1}} u_{0}\right\|_{H^{s}}^{2}\right) \rightarrow 0 \quad \text { as } N_{1} \rightarrow 0 .
\end{aligned}
$$

This shows that $\left\{u^{N}\right\}$ is a Cauchy sequence in $C\left([0,1] ; H^{s}\right)$ and thus $\left\{u^{N}\right\}$ converges in $C\left([0,1] ; H^{s}\right)$ to a solution of (1-3) emanating from $u_{0}$. Note that there is no problem passing to the limit on the nonlinear term here, since we have strong convergence.

Now, Lemma 4.2, Proposition 4.5 and (4-19) ensure that any $L_{1}^{\infty} H^{s}$-solution to (1-3) on ]0, 1[ belongs to $\tilde{Y}_{T}^{s}$. Therefore, according to Propositions 4.7 and $4.8, u$ is the only solution to (1-3) associated with the initial datum $u_{0}$ that belongs to $L_{\text {loc }}^{\infty} H^{s}$.

To prove the continuity of the solution map in $H^{s}(\mathbb{R})$, we proceed as in Section 3B. Let $\left\{u_{0, n}\right\} \subset H^{s}(\mathbb{R})$ be such that $u_{0, n} \rightarrow u_{0}$ in $H^{s}(\mathbb{R})$ and let $\left\{u_{n}\right\} \subset C\left([0,1] ; H^{s}(\mathbb{R})\right)$ be the associated sequence of solutions to (1-3). Taking the same notations as above, we observe that, by construction,

$$
P_{\leq 1}\left(u_{0}-u_{0}^{N}\right)=P_{\leq 1}\left(u_{0, n}-u_{0, n}^{N}\right)=0 \text { for all } N \geq 1 .
$$


This ensures that $u-u^{N}$ and $u_{n}-u_{n}^{N}$ belong to $Z_{T}^{s}$. Estimate (4-31) on solutions to (3-36) then leads to

$$
\left\|u-u^{N}\right\|_{Z_{1}^{s}}+\left\|u_{n}-u_{n}^{N}\right\|_{Z_{1}^{s}} \lesssim\left\|P_{>N} u_{0}\right\|_{H^{s}}+\left\|P_{>N} u_{0, n}\right\|_{H^{s}},
$$

which yields

$$
\lim _{N \rightarrow \infty} \sup _{n \in \mathbb{N}}\left(\left\|u-u^{N}\right\|_{L_{1}^{\infty} H^{s}}+\left\|u_{n}-u_{n}^{N}\right\|_{L_{1}^{\infty} H^{s}}\right)=0 .
$$

It remains to estimate $\left\|u_{n}^{N}-u^{N}\right\|_{H^{s}}$. Note that we cannot use Propositions 4.8 and 4.9 here, since $u_{0, n}^{N}-u_{0}^{N}$ does not belong a priori to $\bar{H}^{s}(\mathbb{R})$. However, since $u_{0}^{N}$ and $u_{0, n}^{N}$ belong to $H^{\infty}(\mathbb{R})$, we know, from the beginning of this section, that $u^{N}$ and $u_{n}^{N}$ belong to $C\left([0,1] ; H^{\infty}(\mathbb{R})\right)$. We now fix $N \gg 1$. Setting $s^{\prime}=\max (1, s)$, we have

$$
\left\|u_{0}^{N}-u_{0, n}^{N}\right\|_{H^{s^{\prime}}} \rightarrow 0 \quad \text { as } n \rightarrow \infty
$$

Therefore, on account of Section 3B,

$$
\left\|u^{N}-u_{n}^{N}\right\|_{L_{T}^{\infty} H^{s^{\prime}}} \rightarrow 0 \quad \text { as } n \rightarrow \infty \quad \text { with } \quad T \sim\left(1+\left\|u_{0}^{N}\right\|_{H^{s^{\prime}}}\right)^{-\frac{2(\alpha+1)}{2 \alpha-1}} .
$$

Since $u^{N} \in C\left([0,1] ; H^{\infty}(\mathbb{R})\right)$ we can iterate this argument a finite number of times to obtain that the convergence of $u_{n}^{N}$ to $u^{N}$ holds actually in $C\left([0,1] ; H^{s^{\prime}}(\mathbb{R})\right)$. The continuity of the flow map in $H^{s}(\mathbb{R})$ follows by combining this last result with (4-32).

4C. The periodic case. We use the notations of Section 3C. Let $H_{0}^{s}(\lambda \mathbb{T})$ be the closed subspace of zero-mean functions of $H_{0}^{s}(\lambda \mathbb{\pi})$. We define the Banach space $\bar{H}^{s}(\lambda \mathbb{\pi})$ as the space $H_{0}^{s}(\lambda \mathbb{\pi})$ endowed with the norm

$$
\|u\|_{\bar{H}^{s}}=\left\|\left\langle|\xi|^{-\frac{1}{2}}\right\rangle\langle\xi\rangle^{s} \hat{\varphi}\right\|_{L_{\xi}^{2}}
$$

Let $(u, v) \in\left(L^{\infty}\left(0, T ; H^{s}(\lambda \mathbb{T})\right)\right)^{2}$ be a pair of solutions to (1-3) associated with initial data $\left(u_{0}, v_{0}\right)$ in $\left(H^{s}(\lambda \mathbb{T})\right)^{2}$ such that $u_{0}-v_{0} \in \bar{H}^{s}(\lambda \mathbb{T})$. As noticed in Remark 1.3, $(u, v) \in C\left([0, T] ; H^{s-\alpha-1}(\lambda \mathbb{T})\right)^{2}$ and it is not too hard to check that the mean value is a constant of the motion for such solutions. Therefore, $u(t)-v(t)$ has mean value zero for all $t \in[0, T]$.

As explained in Section 3C, to extend our result on the torus $\lambda \mathbb{T}$, uniformly for $\lambda \geq 1$, we only have to care about the contributions of the null frequencies each time we used the homogeneous decomposition in space frequencies. First we notice that in the proof of Lemma 4.2 we do not use any homogeneous decomposition in space frequencies and thus this lemma still holds in the periodic setting. Note that this is also true for (4-29), since the proof of this estimate is exactly the same. Moreover, on account of (3-42), the contributions of the null frequencies vanish in the proof of Lemma 4.2. Now, for Propositions 4.7, 4.8 and 4.9, we only have to care about the contributions of $\partial_{x} P_{N}\left(w P_{0} z\right)$, since, according to the discussion above, $P_{0} w=P_{0}(u-v)=0$ on $[0, T]$. On account of (3-43), these contributions vanish in (4-22) and (4-30). Finally, these contributions can be estimated in Proposition 4.7 by

$$
\left\|\partial_{x} P_{N}\left(P_{N} w P_{0} z\right)\right\|_{F^{s-\frac{3}{2}+\frac{\alpha}{2},-\frac{1}{2}}} \lesssim N\left\|P_{N}\left(P_{N} w P_{0} z\right)\right\|_{X^{s-2,0}} \lesssim \delta_{N}\|z\|_{L_{t}^{\infty} L_{x}^{2}}\|w\|_{L_{t}^{2} H_{x}^{s-1}}
$$


with $\left\|\left(\delta_{2^{j}}\right)\right\|_{l^{1}(\mathbb{Z})} \lesssim 1$. This is acceptable, since $1-\frac{\alpha}{2} \geq 0$ and $s-\frac{3}{2}+\frac{\alpha}{2} \geq s-1$. The proof of Theorem 1.5 is now complete.

\section{Dissipative limits}

First, we notice that, if $u$ is a solution to (1-9), then $u_{\lambda}$ defined by $u_{\lambda}(t, x)=\lambda^{\alpha} u\left(\lambda^{1+\alpha} t, \lambda x\right)$ is a solution to

$$
\partial_{t} u_{\lambda}+L_{\alpha+1}^{\lambda} u_{\lambda}+\varepsilon \lambda^{\alpha+1-\beta} A_{\beta}^{\lambda} u_{\lambda}+\frac{1}{2} \partial_{x}\left(u_{\lambda}\right)^{2}=0
$$

with

$$
\widehat{L_{\alpha+1}^{\lambda} v}(\xi)=i \lambda^{\alpha+1} p_{\alpha+1}\left(\lambda^{-1} \xi\right) \hat{v}(\xi)
$$

and

$$
\widehat{A_{\beta}^{\lambda}} v(\xi)=\lambda^{\beta} q_{\beta}\left(\lambda^{-1} \xi\right) \hat{v}(\xi) \text { for all } \xi \in \mathbb{R} .
$$

Therefore, as in the preceding section, up to this change of unknown, of parameter $\varepsilon$ and of operators, we may assume that $u$ satisfies (1-9) with $L_{\alpha+1}$ and $A_{\beta}$ that verify Hypotheses 1 and 2 for all $0<\lambda \leq 1$.

Second, we notice that Hypothesis 2 now ensures that, for $0<\lambda \leq 1$ and $N \gg 1$ dyadic,

$$
\left(A_{\beta}^{\lambda} P_{N} v, P_{N} v\right)_{L^{2}} \gtrsim N^{\frac{\beta}{2}}\left\|P_{N} v\right\|_{L^{2}}^{2}
$$

and

$$
\left\|A_{\beta}^{\lambda} P_{N} v\right\|_{L^{2}} \lesssim N^{\beta}\left\|P_{N} v\right\|_{L^{2}}
$$

The main point is now to prove that the Cauchy problem (1-9) is locally well-posed in $H^{s}$ uniformly in $\varepsilon>0$.

Proposition 5.1. Let $1 \leq \alpha \leq 2,0 \leq \beta \leq 1+\alpha$ and $s \geq 1-\frac{\alpha}{2}$. For any $\varphi \in H^{s}(\mathbb{R})$ there exists $T \sim\left(1+\left\|u_{0}\right\|_{H^{1-\alpha / 2}}\right)^{-2(\alpha+1) /(2 \alpha-1)}$ and a solution $u_{\varepsilon} \in C\left([0, T] ; H^{s}\right)$ to (1-9) that is unique in some function space ${ }^{2}$ embedded in $L_{T}^{\infty}\left(0, T ; H^{s}\right)$. Moreover, there exists $C>0$ such that, for any $\left.\varepsilon \in\right] 0,1[$,

$$
\sup _{t \in[0, T]}\left\|u_{\varepsilon}(t)\right\|_{H^{s}} \leq C\|\varphi\|_{H^{s}}
$$

Finally, for any $R>0$, the family of solution maps $\left.S_{\varepsilon}: \varphi \mapsto u_{\varepsilon}, \varepsilon \in\right] 0,1\left[\right.$, from $B(0, R)_{H^{s}}$ into $C\left([0, T(R)] ; H^{s}(\mathbb{R})\right)$ is equicontinuous, i.e., for any sequence $\left\{\varphi_{n}\right\} \subset B(0, R)_{H^{s}}$ converging to $\varphi$ in $H^{s}(\mathbb{R})$,

$$
\lim _{n \rightarrow+\infty} \sup _{\varepsilon \in] 0,1[}\left\|S_{\varepsilon} \varphi-S_{\varepsilon} \varphi_{n}\right\|_{L^{\infty}\left(0, T(R) ; H^{s}(\mathbb{R})\right)}=0
$$

Proof. We treat the cases $(\alpha, s) \neq\left(1, \frac{1}{2}\right)$. This last case can be treated in the same way by using the estimates derived in the Appendix. First we notice that, for (1-9), in view of (5-2), the energy estimate (4-14) becomes

$$
\|u\|_{\widetilde{L}_{T}^{\infty} H^{s}}+\sqrt{\varepsilon}\|u\|_{L_{T}^{2} H^{s+\frac{\beta}{2}}} \lesssim\left\|u_{0}\right\|_{H^{s}}+\|u\|_{L_{T}^{\infty} H^{1-\frac{\alpha}{2}}}\|u\|_{Y_{T}^{s}}+\|u\|_{L_{T}^{\infty} H^{s}}\|u\|_{Y_{T}^{1-\frac{\alpha}{2}}} .
$$

\footnotetext{
${ }^{2}$ For $(\alpha, s) \neq\left(1, \frac{1}{2}\right)$, this space is simply the space $L_{T}^{\infty} H^{s} \cap L_{T}^{2} H^{s+\beta / 2}$.
} 
On the other hand, viewing $\varepsilon A_{\beta} u$ as a forcing term, (4-2)-(4-3) together with (5-3) lead to

$$
\|u\|_{Y_{T}^{s}} \lesssim\|u\|_{L_{T}^{\infty} H^{s}}\left(1+\|u\|_{L_{T}^{\infty} H^{1-\frac{\alpha}{2}}}^{2}\right)+\varepsilon\|u\|_{L_{T}^{2} H^{s-\frac{1+\alpha}{2}+\beta}} .
$$

To derive an a priori bound from the above estimates, as in the previous section, we have to use the dilation argument that is described in the beginning of this section. So the dilation function $u_{\lambda}$ defined by $u_{\lambda}(t, x)=\lambda^{\alpha} u\left(\lambda^{1+\alpha} t, \lambda x\right)$ satisfies (5-1) and we set

$$
\|v\|_{N^{s}}:=\|v\|_{L_{T}^{\infty} H^{s}}+\sqrt{\varepsilon \lambda^{\alpha+1-\beta}}\|v\|_{L_{T}^{2} H^{s+\frac{\beta}{2}}} .
$$

Since $\beta \leq \alpha+1$, this ensures that, for $\lambda \lesssim\left(1+\|\varphi\|_{H^{s}}\right)^{-2(\alpha+1) /(2 \alpha-1)}$ and $0<T \leq 2$,

$$
\left\|u_{\lambda}\right\|_{N_{T}^{s}} \lesssim\left\|\varphi_{\lambda}\right\|_{H^{s}}+\left(1+\left\|u_{\lambda}\right\|_{N_{T}^{1-\frac{\alpha}{2}}}^{2}\right)\left\|u_{\lambda}\right\|_{N_{T}^{1-\frac{\alpha}{2}}}\left\|u_{\lambda}\right\|_{N_{T}^{s}}
$$

with $\left\|\varphi_{\lambda}\right\|_{H^{s}} \lesssim \lambda^{\alpha-1 / 2}\|\varphi\|_{H^{s}} \ll 1$. This leads to the uniform bound (5-4) for smooth solutions to (1-9) by a classical continuity argument.

Now, proceeding in the same way for the difference of two solutions, it is not too hard to check that (4-20) becomes

$$
\begin{aligned}
& \|u-v\|_{Z_{T}^{s-\frac{3}{2}+\frac{\alpha}{2}}} \\
& \lesssim\|u-v\|_{\tilde{L}_{T}^{\infty} \bar{H}^{s-\frac{3}{2}+\frac{\alpha}{2}}}+\|u-v\|_{L_{T}^{2} H^{s-\frac{3}{2}+\frac{\alpha}{2}+\beta}}+\|u+v\|_{\widetilde{Y}_{T}^{s}}\|u-v\|_{Z_{T}^{-\frac{1}{2}}}+\|u+v\|_{\widetilde{Y}^{1-\frac{\alpha}{2}}}\|u-v\|_{Z_{T}^{s-\frac{3}{2}+\frac{\alpha}{2}}},
\end{aligned}
$$

whereas (4-22) becomes

$$
\|u-v\|_{\widetilde{L}_{T}^{\infty} \bar{H}^{s-\frac{3}{2}+\frac{\alpha}{2}}}+\sqrt{\varepsilon}\|u-v\|_{L_{T}^{2} \bar{H}^{s-\frac{3}{2}+\frac{\alpha}{2}+\frac{\beta}{2}}} \lesssim\left\|u_{0}-v_{0}\right\|_{\bar{H}^{s-\frac{3}{2}+\frac{\alpha}{2}}}+\|u+v\|_{\tilde{Y}_{T}^{s}}\|u-v\|_{Z_{T}^{s-\frac{3}{2}+\frac{\alpha}{2}}} .
$$

By the same dilation arguments as above, this leads to

$$
\|u-v\|_{Z_{T}^{s-\frac{3}{2}+\frac{\alpha}{2}}}+\sqrt{\varepsilon}\|u-v\|_{L_{T}^{2} H^{s-\frac{3}{2}+\frac{\alpha}{2}+\frac{\beta}{2}}} \lesssim\left\|u_{0}-v_{0}\right\|_{\bar{H}^{s-\frac{3}{2}+\frac{\alpha}{2}}} .
$$

Combining the above estimates and the Bona-Smith argument, we can proceed as in Section 4B and construct a sequence of smooth solutions that converges strongly in $C\left([0, T] ; H^{s}\right)$ towards a solution $u_{\varepsilon}$ to (1-9). We thus obtain the existence of a solution $u_{\varepsilon} \in C\left([0, T] ; H^{s}\right) \cap L_{T}^{2} H^{s+\beta / 2}$ to (1-9) with $T \gtrsim\left(1+\left\|u_{0}\right\|_{H^{1-\alpha / 2}}\right)^{-2(\alpha+1) /(2 \alpha-1)}$ and $\varphi \in H^{s}$ as initial data. Moreover, (5-8) ensures that this is the only solution emanating from $\varphi$ in the class $L_{\mathrm{loc}}^{\infty} H^{s} \cap L_{\mathrm{loc}}^{2} H^{s+\beta / 2}$. Obviously, this solution satisfies (5-4). Finally, the equicontinuity of the solution map in $C\left(0, T ; H^{S}\right)$ follows from Bona-Smith arguments as in Section 3B.

It is clear that the above proposition implies part (1) of Theorem 1.14. Now, part (2) will follow from general arguments (see for instance [Guo and Wang 2009]). Let us denote by $S_{\varepsilon}$ and $S$ the nonlinear group associated with, respectively, (1-9) and (1-3). Let $\varphi \in H^{s}(\mathbb{R}), s \geq 1-\frac{\alpha}{2}$ and let $T=T\left(\|\varphi\|_{H^{1-\alpha / 2}}\right)>0$ 
be as given by Proposition 5.1. For any $N>0$ we can rewrite $S_{\varepsilon}(\varphi)-S(\varphi)$ as

$$
\begin{aligned}
S_{\varepsilon}(\varphi)-S(\varphi) & =\left(S_{\varepsilon}(\varphi)-S_{\varepsilon}\left(P_{\leq N} \varphi\right)\right)+\left(S_{\varepsilon}\left(P_{\leq N} \varphi\right)-S\left(P_{\leq N} \varphi\right)\right)+\left(S\left(P_{\leq N} \varphi\right)-S(\varphi)\right) \\
& =I_{\varepsilon, N}+J_{\varepsilon, N}+K_{N} .
\end{aligned}
$$

By continuity with respect to initial data in $H^{s}(\mathbb{R})$ of the solution map associated with (1-3), we have $\lim _{N \rightarrow \infty}\left\|K_{N}\right\|_{L^{\infty}\left(0, T ; H^{s}\right)}=0$. Moreover, (5-5) ensures that

$$
\lim _{N \rightarrow \infty} \sup _{\varepsilon \in] 0,1[}\left\|I_{\varepsilon, N}\right\|_{L^{\infty}\left(0, T ; H^{s}\right)}=0 .
$$

It thus remains to check that, for any fixed $N>0, \lim _{\varepsilon \rightarrow 0}\left\|J_{\varepsilon, N}\right\|_{L^{\infty}\left(0, T ; H_{x}^{s}\right)}=0$. Since $P_{\leq N} \varphi \in H^{\infty}(\mathbb{R})$, it is worth noticing that $S_{\varepsilon}\left(P_{\leq N} \varphi\right)$ and $S\left(P_{\leq N} \varphi\right)$ belong to $C^{\infty}\left(\mathbb{R} ; H^{\infty}(\mathbb{R})\right)$. Moreover, according to Theorem 1.14 and Proposition 5.1, for all $\theta \in \mathbb{R}$ and $\varepsilon \in] 0,1[$,

$$
\left\|S_{\varepsilon}\left(P_{\leq N} \varphi\right)\right\|_{L_{T}^{\infty} H_{x}^{\theta}}+\left\|S\left(P_{\leq N} \varphi\right)\right\|_{L_{T}^{\infty} H_{x}^{\theta}} \leq C\left(N, \theta,\|\varphi\|_{L_{x}^{2}}\right) .
$$

Now, setting $v_{\varepsilon}:=S_{\varepsilon}\left(P_{\leq N} \varphi\right)$ and $v:=S\left(P_{\leq N} \varphi\right)$, we observe that $w_{\varepsilon}:=v_{\varepsilon}-v$ satisfies

$$
\partial_{t} w_{\varepsilon}+L_{\alpha+1} w_{\varepsilon}=-\frac{1}{2} \partial_{x}\left(w_{\varepsilon}\left(v+v_{\varepsilon}\right)\right)-\varepsilon A_{\beta} v_{\varepsilon}
$$

with initial data $w_{\varepsilon}(0)=0$. For $s \geq 0$, taking the $H^{s}$-scalar product of this last equation with $w_{\varepsilon}$ and integrating by parts, we get

$$
\frac{d}{d t}\left\|w_{\varepsilon}\right\|_{H^{s}} \lesssim\left(1+\left\|\partial_{x}\left(v+v_{\varepsilon}\right)\right\|_{\left.L_{x}^{\infty}\right)}\left\|w_{\varepsilon}\right\|_{H^{s}}^{2}+\left\|\left[J^{s} \partial_{x},\left(v+v_{\varepsilon}\right)\right] w_{\varepsilon}\right\|_{L_{x}^{2}}\left\|w_{\varepsilon}\right\|_{H^{s}}+\varepsilon^{2}\left\|D_{x}^{\beta} v_{\varepsilon}\right\|_{H^{s}}^{2} .\right.
$$

Applying the mean value theorem to the Fourier transform of the commutator term, it is not too hard to check that

$$
\left\|\left[J_{x}^{s} \partial_{x}, f\right] g\right\|_{L_{x}^{2}} \lesssim\left\|f_{x}\right\|_{H^{s+1}}\|g\|_{H_{x}^{s}},
$$

which leads to

$$
\frac{d}{d t}\left\|w_{\varepsilon}(t)\right\|_{H^{s}}^{2} \lesssim C\left(N, s+2,\|\varphi\|_{L_{x}^{2}}\right)\left\|w_{\varepsilon}(t)\right\|_{H_{x}^{s}}^{2}+\varepsilon^{2} C\left(N, s+\beta,\|\varphi\|_{L_{x}^{2}}\right)^{2} .
$$

Integrating this differential inequality on $[0, T]$, this ensures that $\lim _{\varepsilon \rightarrow 0}\left\|w_{\varepsilon}\right\|_{L^{\infty}\left(0, T ; H^{s}\right)}=0$ and proves that

$$
u_{\varepsilon} \rightarrow u \quad \text { in } C\left([0, T] ; H^{s}\right)
$$

with $T \sim\left(1+\left\|u_{0}\right\|_{H^{1-\alpha / 2}}\right)^{-2(\alpha+1) /(2 \alpha-1)}$. Now fix $\varphi \in H^{s}$ and let $T^{*}>0$ be the maximal time of existence of $S(\varphi)$. It remains to prove that the time of existence $T_{\varepsilon}$ of $S_{\varepsilon}(\varphi)$ in $H^{s}$ satisfies $\liminf _{\varepsilon \rightarrow 0} T_{\varepsilon} \geq T^{*}$. Actually, this follows by a classical contradiction argument. Indeed, assuming that this is not true, there exist $\varepsilon_{n} \searrow 0$ such that $\lim T_{\varepsilon_{n}}=T_{1}<T^{*}$. We set

$$
\delta\left(T_{1}\right)=\left(1+\|S(\varphi)\|_{L^{\infty}\left(0, T_{1} ; H^{1-\frac{\alpha}{2}}\right)}\right)^{-\frac{2(\alpha+1)}{2 \alpha-1}},
$$


which is well defined since $T_{1}<T^{*}$. Applying (5-10) about $T_{1} / \delta\left(T_{1}\right)$ times, we eventually obtain that, for $n$ large enough,

$$
\left\|S_{\varepsilon_{n}}(\varphi)\left(T_{1}-\frac{1}{100} \delta\left(T_{1}\right)\right)\right\|_{H^{1-\frac{\alpha}{2}}} \leq 2\|S(\varphi)\|_{L^{\infty}\left(0, T_{1} ; H^{1-\frac{\alpha}{2}}\right)} .
$$

But then the uniform bound from below on the existence time ensures that $T_{\varepsilon_{n}} \geq T_{1}+\frac{1}{2} \delta\left(T_{1}\right)$, which contradicts $\lim T_{\varepsilon_{n}}=T_{1}$ and proves the desired result. This ensures that, fixing $0<T_{0}<T^{*}$, we have $T_{\varepsilon} \geq T_{0}$ for $\varepsilon>0$ small enough. Finally, applying (5-10) about $T_{0} / \delta\left(T_{0}\right)$ times, we get (5-10) with $T=T_{0}$. This completes the proof of Theorem 1.14.

\section{Appendix: The case $\alpha=1$ and $s=\frac{1}{2}$}

This case is important since $H^{1 / 2}$ is the energy space for the Benjamin-Ono equation and also the intermediate long waves equation. Unfortunately, we are not able to prove the unconditional wellposedness in this case. However, we are able to prove the well-posedness without using a gauge transform. This is useful for treating perturbations of these equations, as we explained in the preceding section. In this section, we indicate the modifications of the proofs in this case. In the sequel we set

$$
\tilde{M}^{\frac{1}{2}}:=\widetilde{L}_{t}^{\infty} H^{\frac{1}{2}} \cap X^{-\frac{1}{2}, 1} .
$$

Lemma A.1. Let $\alpha=1,0<T<2$, and let $u \in \tilde{M}_{T}^{1 / 2}$ be a solution to (1-3). Then

$$
\|u\|_{\tilde{M}_{T}^{\frac{1}{2}}} \lesssim\|u\|_{\tilde{L}_{T}^{\infty} H^{\frac{1}{2}}}+\|u\|_{\tilde{M}_{T}^{\frac{1}{2}}}^{2} .
$$

Proof. Working with the extension $\tilde{u}=\rho_{T} u$ (see (3-3)), still denoted $u$, if suffices to estimate the $X^{-1 / 2,1}$-norm of $u$. First we notice that the low frequency part can be easily controlled by

Now, for $N \gg 1$, we have

$$
\left\|P_{\lesssim 1} u\right\|_{X_{T}^{-\frac{1}{2}, 1}} \lesssim\|u\|_{L_{T}^{\infty} L_{x}^{2}}^{2} .
$$

$$
\begin{aligned}
\left\|u_{N}\right\|_{X_{T}^{-\frac{1}{2}, 1}} \lesssim\left\|P_{N} u_{0}\right\|_{H^{-\frac{1}{2}}}+N^{\frac{1}{2}}\left\|\sum_{N_{2}^{\prime} \sim N_{2} \gtrsim N} u_{N_{2}} u_{N_{2}^{\prime}}\right\|_{L_{T}^{2} L_{x}^{2}} \\
\quad+N^{\frac{1}{2}}\left\|\sum_{1 \leq N_{2} \ll N} P_{N}\left(u_{\sim N} u_{N_{2}}\right)\right\|_{L_{T}^{2} L_{x}^{2}}+N^{\frac{1}{2}}\left\|\sum_{N_{2}<1} P_{N}\left(u_{\sim N} u_{N_{2}}\right)\right\|_{L_{T}^{2} L_{x}^{2}} \\
=\left\|P_{N} u_{0}\right\|_{H^{-\frac{1}{2}}}+I_{N}+I I_{N}+I I I_{N} .
\end{aligned}
$$

Clearly,

$$
\begin{aligned}
I_{N} & \lesssim N^{\frac{1}{2}} \sum_{N_{2}^{\prime} \sim N_{2} \gtrsim N}\left\|u_{N_{2}}\right\|_{L_{t x}^{2}}\left\|u_{N_{2}^{\prime}}\right\|_{L_{t}^{\infty} H^{\frac{1}{2}}} \\
& \lesssim\|u\|_{L_{t}^{\infty} H^{\frac{1}{2}}} \sum_{N_{2} \gtrsim N}\left(\frac{N}{N_{2}}\right)^{\frac{1}{2}}\left\|u_{N_{2}}\right\|_{L_{t}^{2} H^{\frac{1}{2}}} \\
& \lesssim \delta_{N}\|u\|_{L_{t}^{\infty} H^{\frac{1}{2}}}^{2}
\end{aligned}
$$


with $\left\|\left(\delta_{2^{j}}\right)\right\|_{l^{2}\left(\mathbb{N}^{*}\right)} \lesssim 1$. Moreover, we easily get from Bernstein estimates that

$$
I I I_{N} \lesssim N^{\frac{1}{2}} \sum_{N_{2}<1}\|u \sim N\|_{L_{t x}^{2}}\left\|u_{N_{2}}\right\|_{L_{t x}^{\infty}} \lesssim\left\|u_{\sim N}\right\|_{L_{t}^{2} H^{\frac{1}{2}}}\|u\|_{L_{t}^{\infty} H^{\frac{1}{2}}} \lesssim \delta_{N}\|u\|_{L_{t}^{\infty} H^{\frac{1}{2}}}\|u\|_{L_{t}^{\infty} H^{\frac{1}{2}}}
$$

with $\left\|\left(\delta_{2^{j}}\right)\right\|_{l^{2}\left(\mathbb{N}^{*}\right)} \lesssim 1$. On the other hand,

$$
I I_{N} \lesssim N^{\frac{1}{2}}\left\|\sum_{1 \leq N_{2} \ll N} Q_{\sim N N_{2}} P_{N}\left(u_{\sim N} u_{N_{2}}\right)\right\|_{L_{t x}^{2}}+N^{\frac{1}{2}}\left\|\sum_{1 \leq N_{2} \ll N} Q_{\nsim N N_{2}} P_{N}\left(u \sim N u_{N_{2}}\right)\right\|_{L_{t x}^{2}} \lesssim I I_{N}^{1}+I I_{N}^{2} .
$$

By almost orthogonality, we have

$$
\begin{aligned}
I I_{N}^{1} & \lesssim N^{\frac{1}{2}}\left(\sum_{1 \leq N_{2} \ll N}\left\|Q_{\sim N N_{2}} P_{N}\left(u_{\sim N} u_{N_{2}}\right)\right\|_{L_{t x}^{2}}^{2}\right)^{\frac{1}{2}} \\
& \lesssim N^{\frac{1}{2}}\left(\sum_{N_{2} \ll N}\left\|u_{\sim N}\right\|_{L_{t x}^{2}}^{2}\left\|u_{N_{2}}\right\|_{L_{t}^{\infty} H_{x}^{\frac{1}{2}}}^{2}\right)^{\frac{1}{2}} \\
& \lesssim\|u \sim N\|_{L_{t}^{2} H^{\frac{1}{2}}}\|u\|_{\tilde{L}_{t}^{\infty} H^{\frac{1}{2}}} \\
& \lesssim \delta_{N}\|u\|_{L_{t}^{\infty} H^{\frac{1}{2}}}\|u\|_{\tilde{L}_{t}^{\infty} H^{\frac{1}{2}}}
\end{aligned}
$$

with $\left\|\left(\delta_{2^{j}}\right)\right\|_{l^{2}\left(\mathbb{N}^{*}\right)} \lesssim 1$. It remains to control $I I_{N}^{2}$. Since the Fourier projectors ensure $\left\langle\tau-p_{2}(\xi)\right\rangle \nsim N_{2}$, the resonance relation (1-6) leads to $\left.\left|\tau_{1}-p_{2}\left(\xi_{1}\right)\right| \vee\left|\tau-\tau_{1}-p_{2}\left(\xi-\xi_{1}\right)\right|\right) \gtrsim N N_{2}$ for $I I_{N}^{2}$. We separate the contributions of $Q_{\gtrsim N N_{2}} u \sim N$ and $Q_{\gtrsim N N_{2}} u_{N_{2}}$. For the first contribution, we have

$$
\begin{aligned}
I I_{N}^{2} & \lesssim N^{\frac{1}{2}} \sum_{1 \leq N_{2} \ll N}\left(N N_{2}\right)^{-\frac{1}{4}} N^{\frac{1}{4}}\left\|Q_{\gtrsim N N_{2}} u \sim N\right\|_{X^{\frac{1}{4}, \frac{1}{4}}}\left\|u_{N_{2}}\right\|_{L_{t}^{\infty} H^{\frac{1}{2}}} \\
& \lesssim\|u \sim N\|_{X^{\frac{1}{4}, \frac{1}{4}}}\|u\|_{L_{t}^{\infty} H^{\frac{1}{2}}} \\
& \lesssim \delta_{N}\|u\|_{X^{-\frac{1}{2}, 1}}^{\frac{1}{4}}\|u\|_{L_{t}^{\infty} H^{\frac{1}{2}}}^{\frac{3}{4}}\|u\|_{L_{t}^{\infty} H^{\frac{1}{2}}}
\end{aligned}
$$

with $\left\|\left(\delta_{2^{j}}\right)\right\|_{l^{2}\left(\mathbb{N}^{*}\right)} \lesssim 1$ and where we used interpolation at the last step. For the second contribution, we write

$$
\begin{aligned}
I I_{N}^{2} & \lesssim N^{\frac{1}{2}} \sum_{1 \leq N_{2} \ll N}\left\|Q_{\ll N N_{2}} u \sim N\right\|_{L_{t}^{\infty} L_{x}^{4}}\left\|Q_{\gtrsim N N_{2}} u_{N_{2}}\right\|_{L_{t}^{2} L_{x}^{4}} \\
& \lesssim N^{\frac{1}{2}} \sum_{1 \leq N_{2} \ll N} N^{-\frac{1}{4}}\left\|Q_{\ll N N_{2}} u \sim N\right\|_{L_{t}^{\infty} H^{\frac{1}{2}}}\left\|Q_{\gtrsim N N_{2}} u_{N_{2}}\right\|_{L_{t}^{2} H^{\frac{1}{4}}} \\
& \lesssim N^{\frac{1}{2}} \sum_{1 \leq N_{2} \ll N} N^{-\frac{1}{4}}\left(N N_{2}\right)^{-\frac{1}{4}}\|u \sim N\|_{L_{t}^{\infty} H^{\frac{1}{2}}}\left\|u_{N_{2}}\right\|_{X^{\frac{1}{4}, \frac{1}{4}}} \\
& \lesssim \delta_{N}\|u\|_{\tilde{L}_{t}^{\infty} H^{\frac{1}{2}}}\|u\|_{X^{-\frac{1}{2}, 1}}^{\frac{1}{4}}\|u\|_{L_{t}^{\infty} H^{\frac{1}{2}}}^{\frac{3}{4}}
\end{aligned}
$$

with $\left\|\left(\delta_{2^{j}}\right)\right\|_{l^{2}\left(\mathbb{N}^{*}\right)} \lesssim 1$. Gathering the above estimates, (5-2) follows. 
Lemma A.2. Let $\alpha=1,0<T<2$ and let $u \in \tilde{M}_{T}^{1 / 2}$ be a solution to (1-3). Then

$$
\|u\|_{\tilde{L}_{T}^{\infty} H^{\frac{1}{2}}}^{2} \lesssim\left\|u_{0}\right\|_{H^{\frac{1}{2}}}^{2}+\|u\|_{\tilde{L}_{t}^{\infty} H^{\frac{1}{2}}}^{2}\|u\|_{\tilde{M}_{T}^{\frac{1}{2}}} \cdot
$$

Proof. We follow the proof of Proposition 4.5. Note that $\tilde{M}^{1 / 2} \hookrightarrow \widetilde{Y}^{1 / 2}$. According to (4-15), it suffices to control

$$
I=\sum_{N>0} \sum_{N_{1} \gtrsim N} N\left\langle N_{1}\right\rangle \sup _{t \in] 0, T[}\left|I_{t}\left(u_{N}, u_{\sim N_{1}}, u_{N_{1}}\right)\right|
$$

It is easy to check that the only term of the left-hand side of (4-16) that causes trouble in the case $\alpha=1$ is

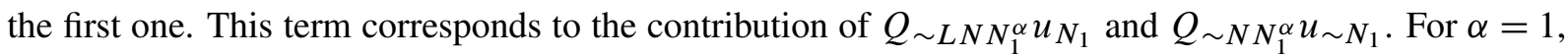
we control these contributions by applying Cauchy-Schwarz in $\left(N, N_{1}\right)$. For instance, the contribution of $Q \sim L N N_{1}^{\alpha} u_{N_{1}}$ is estimated, thanks to Lemma 4.4, by

$$
\begin{array}{rl}
\sum_{N \gg 1} \sum_{N_{1} \gtrsim N} & N\left\langle N_{1}\right\rangle \sum_{L>1} L^{-1} N^{-\frac{1}{2}}\left\|u_{N}\right\|_{L_{t x}^{2}}\left\|Q_{\sim L N N_{1}^{\alpha}} u_{N_{1}}\right\|_{F^{0, \frac{1}{2}}}\left\|u_{\sim N_{1}}\right\|_{L_{t}^{\infty} L_{x}^{2}} \\
& \lesssim \sum_{L>1} L^{-1}\left(\sum_{N_{1} \gtrsim N \gg 1}\left\|u_{N}\right\|_{L_{t}^{2} H^{\frac{1}{2}}}^{2}\left\|u_{\sim N_{1}}\right\|_{L_{t}^{\infty} H^{\frac{1}{2}}}^{2}\right)^{\frac{1}{2}}\left(\sum_{N_{1} \gtrsim N \gg 1}\left\|Q_{\sim L N N_{1}^{\alpha}} u_{N_{1}}\right\|_{F^{\frac{1}{2}, \frac{1}{2}}}^{2}\right)^{\frac{1}{2}} \\
& \lesssim\|u\|_{L_{t}^{2} H^{\frac{1}{2}}}\|u\|_{\tilde{L}_{t}^{\infty} H^{\frac{1}{2}}}\|u\|_{X^{-\frac{1}{2}, 1}}
\end{array}
$$

Lemma A.3. Let $0<T<2$ and let $u, v \in \widetilde{M}_{T}^{1 / 2}$ be two solutions to (1-3) on $] 0, T$. Then we have

$$
\|u-v\|_{Z_{T}^{-\frac{1}{2}}} \lesssim\|u-v\|_{L_{T}^{\infty} \bar{H}^{s-\frac{3}{2}+\frac{\alpha}{2}}}+\|u+v\|_{\tilde{M}_{T}^{\frac{1}{2}}}\|u-v\|_{Z_{T}^{-\frac{1}{2}}}
$$

and

$$
\|u-v\|_{\tilde{L}_{T}^{\infty} \bar{H}^{-\frac{1}{2}}}^{2} \lesssim\left\|u_{0}-v_{0}\right\|_{\bar{H}^{-\frac{1}{2}}}^{2}+\|u+v\|_{\tilde{M}_{T}^{\frac{1}{2}}}\|u-v\|_{\tilde{L}_{T}^{\infty} \bar{H}^{-\frac{1}{2}}}\|u-v\|_{Z_{T}^{-\frac{1}{2}}} \cdot
$$

Proof. First we notice that (A-4) is already proven in Proposition 4.8, since $\tilde{M}_{T}^{1 / 2} \hookrightarrow \tilde{Y}_{T}^{1 / 2} \hookrightarrow Y_{T}^{1 / 2}$. It remains to prove (A-3). We follow the proof of Proposition 4.5. It is not too hard to check that the only contribution that causes troubles in the right-hand side of (4-21), in the case $\alpha=1$, is the contribution of the low-high interaction term, $P_{N}\left(P_{\lesssim N} z w_{N}\right)$. We proceed as in Lemma A.1 . We take extensions $\tilde{z}$ and $\tilde{w}$, supported in $]-4,4\left[\right.$, of $z$ and $w$ such that $\|\tilde{z}\|_{\tilde{M}^{1 / 2}} \lesssim\|z\|_{\tilde{M}_{T}^{1 / 2}}$ and $\|\widetilde{w}\|_{Z^{-1 / 2}} \lesssim\|w\|_{Z_{T}^{-1 / 2}}$. For simplicity we drop the tilde. We first notice that the contribution of $P_{\lesssim 1} z$ is easily estimated by

$$
\left\|\partial_{x} P_{N}\left(P_{\lesssim 1} z w_{\sim N}\right)\right\|_{F^{-\frac{1}{2}, 1,-\frac{1}{2}}} \lesssim\langle N\rangle^{-\frac{1}{2}}\left\|P_{N}\left(P_{\lesssim 1} z w_{\sim N}\right)\right\|_{L_{t x}^{2}} \lesssim\|z\|_{L_{t}^{\infty} L_{x}^{2}}\left\|w_{\sim N}\right\|_{L_{t}^{2} H^{-\frac{1}{2}}},
$$


which is acceptable. Now we decompose the remaining contribution as

$$
\begin{aligned}
& \left\|\partial_{x} P_{N}\left(P_{\gg 1} P_{\lesssim N} z w_{\sim N}\right)\right\|_{F^{-\frac{1}{2}, 1,-\frac{1}{2}}} \\
& \quad \lesssim N\left\|\sum_{1 \ll N_{1} \lesssim N} P_{N}\left(P_{N_{1}} z w_{\sim N}\right)\right\|_{X^{-\frac{3}{2}, 0}} \\
& \quad \lesssim\langle N\rangle^{-\frac{1}{2}}\left\|_{1 \ll N_{1} \lesssim N} Q_{\sim N N_{1}} P_{N}\left(P_{N_{1}} z w_{\sim N}\right)\right\|_{L_{t x}^{2}}+\langle N\rangle^{-\frac{1}{2}}\left\|_{1 \ll N_{1} \lesssim N} Q_{\sim N N_{1}} P_{N}\left(P_{N_{1}} z w_{\sim N}\right)\right\|_{L_{t x}^{2}} \\
& \quad=J_{1, N}+J_{2, N} .
\end{aligned}
$$

By almost-orthogonality,

$$
\begin{aligned}
J_{1, N} & \lesssim\langle N\rangle^{-\frac{1}{2}}\left(\sum_{1 \ll N_{1} \lesssim N}\left\|Q_{\sim N N_{1}} P_{N}\left(P_{N_{1}} z w_{\sim N}\right)\right\|_{L_{t x}^{2}}^{2}\right)^{\frac{1}{2}} \\
& \lesssim\langle N\rangle^{-\frac{1}{2}}\left(\sum_{1 \ll N_{1} \lesssim N}\left\|P_{N_{1}} z\right\|_{L_{t}^{2} H^{\frac{1}{2}}}^{2}\left\|w_{\sim N}\right\|_{L_{t x}^{\infty}}^{2}\right)^{\frac{1}{2}} \\
& \lesssim\left\|w_{\sim N}\right\|_{L_{t}^{\infty} H^{-\frac{1}{2}}\|z\|_{L_{t}^{2} H^{\frac{1}{2}}}}
\end{aligned}
$$

which is acceptable. To treat $J_{2}$, we notice that, since the Fourier projectors ensure that $\left\langle\tau-p_{2}(\xi)\right\rangle \nsim N N_{1}$, the resonance relation (1-6) leads to $\left|\tau_{1}-p_{2}\left(\xi_{1}\right)\right| \vee\left|\tau-\tau_{1}-p_{2}\left(\xi-\xi_{1}\right)\right| \gtrsim N N_{1}$ for $J_{2, N}$. We separate the contributions of $Q_{\gtrsim N N_{1}} z_{N_{1}}$ and $Q_{\gtrsim N N_{1}} w_{\sim N}$. For the first contribution, we write

$$
\begin{aligned}
J_{2, N} & \lesssim\langle N\rangle^{-\frac{1}{2}} \sum_{1 \ll N_{1} \lesssim N} N_{1}^{\frac{1}{2}}\left\|Q_{\gtrsim N N_{1}} P_{N_{1}} z\right\|_{L_{t x}^{2}}\left\|w_{\sim N}\right\|_{L_{t}^{\infty} L_{x}^{2}} \\
& \lesssim\langle N\rangle^{-\frac{1}{2}} \sum_{1 \ll N_{1} \lesssim N}\left(N N_{1}\right)^{-\frac{1}{4}} N_{1}^{\frac{1}{4}}\left\|Q_{\gtrsim N N_{1}} P_{N_{1}} z\right\|_{X^{\frac{1}{4}, \frac{1}{4}}}\left\|w_{\sim N}\right\|_{L_{t}^{\infty} L_{x}^{2}} \\
& \lesssim\|z\|_{X^{-\frac{1}{2}, 1}}^{\frac{1}{4}}\|z\|_{L_{t}^{\infty} H^{\frac{1}{2}}}^{\frac{3}{4}}\left\|w_{\sim N}\right\|_{L_{t}^{\infty} H^{-\frac{1}{2}}},
\end{aligned}
$$

which is acceptable. For the second contribution, according to (4-10), we have

$$
\begin{aligned}
J_{2} & \lesssim\langle N\rangle^{-\frac{1}{2}} \sum_{1 \ll N_{1} \lesssim N}\left\|z_{N_{1}}\right\|_{L_{t}^{\infty} H^{\frac{1}{2}}}\left\|Q_{\gtrsim N N_{1}} w_{\sim N}\right\|_{L_{t x}^{2}} \\
& \lesssim\langle N\rangle^{-\frac{1}{2}} \sum_{1 \ll N_{1} \lesssim N}\left(N N_{1}\right)^{-1} N^{\frac{3}{2}}\left\|z_{N_{1}}\right\|_{L_{t}^{\infty} H^{\frac{1}{2}}}\left\|w_{\sim N}\right\|_{F^{-\frac{1}{2}, \frac{1}{2}}} \\
& \lesssim\left\|w_{\sim N}\right\|_{F^{-\frac{1}{2}, \frac{1}{2}}}\|z\|_{L_{t}^{\infty} H^{\frac{1}{2}}}
\end{aligned}
$$

which is acceptable. Gathering the above estimates we obtain (A-3).

Gathering Lemmas A.1-A.3 and proceeding as in Section 4B we obtain the local well-posedness in $H^{1 / 2}$ of (1-3) for $\alpha=1$. Note that the uniqueness holds in the space $\widetilde{M}_{T}^{1 / 2}$. 


\section{Acknowledgements}

The authors were partially supported by the ANR project GEODISP. They would like to thank the anonymous referee for his careful reading and valuable comments that allowed us to significantly improve the first version of this work.

\section{References}

[Abdelouhab et al. 1989] L. Abdelouhab, J. L. Bona, M. Felland, and J.-C. Saut, "Nonlocal models for nonlinear, dispersive waves", Phys. D 40:3 (1989), 360-392. MR 91d:58033 Zbl 0737.35102

[Adams 1975] R. A. Adams, Sobolev spaces, Pure and Applied Mathematics 65, Academic Press, New York, 1975. MR 56 \#9247 Zbl 0314.46030

[Babin et al. 2011] A. V. Babin, A. A. Ilyin, and E. S. Titi, "On the regularization mechanism for the periodic Korteweg-de Vries equation”, Comm. Pure Appl. Math. 64:5 (2011), 591-648. MR 2012d:35313 Zbl 1284.35365

[Bona and Smith 1975] J. L. Bona and R. Smith, "The initial-value problem for the Korteweg-de Vries equation", Philos. Trans. Roy. Soc. London Ser. A 278:1287 (1975), 555-601. MR 52 \#6219 Zbl 0306.35027

[Bourgain 1993a] J. Bourgain, "Fourier transform restriction phenomena for certain lattice subsets and applications to nonlinear evolution equations, II: The KdV-equation", Geom. Funct. Anal. 3:3 (1993), 209-262. MR 95d:35160b Zbl 0787.35098

[Bourgain 1993b] J. Bourgain, "On the Cauchy problem for the Kadomtsev-Petviashvili equation”, Geom. Funct. Anal. 3:4 (1993), 315-341. MR 94d:35142 Zbl 0787.35086

[Burq and Planchon 2008] N. Burq and F. Planchon, "On well-posedness for the Benjamin-Ono equation", Math. Ann. 340:3 (2008), 497-542. MR 2009a:35205 Zbl 1148.35074

[Colliander et al. 2004] J. Colliander, M. Keel, G. Staffilani, H. Takaoka, and T. Tao, "Multilinear estimates for periodic KdV equations, and applications", J. Funct. Anal. 211:1 (2004), 173-218. MR 2005a:35241 Zbl 1062.35109

[Edwin and Roberts 1986] P. M. Edwin and B. Roberts, "The Benjamin-Ono-Burgers equation: an application in solar physics", Wave Motion 8:2 (1986), 151-158. MR 87d:76154 Zbl 0587.76193

[Guo 2012] Z. Guo, "Local well-posedness for dispersion generalized Benjamin-Ono equations in Sobolev spaces", J. Differential Equations 252:3 (2012), 2053-2084. MR 2860610 Zbl 1298.35099

[Guo and Wang 2009] Z. Guo and B. Wang, "Global well-posedness and inviscid limit for the Korteweg-de Vries-Burgers equation”, J. Differential Equations 246:10 (2009), 3864-3901. MR 2010e:35235 Zbl 1170.35084

[Guo et al. 2011] Z. Guo, L. Peng, B. Wang, and Y. Wang, "Uniform well-posedness and inviscid limit for the Benjamin-OnoBurgers equation”, Adv. Math. 228:2 (2011), 647-677. MR 2012h:35305 Zbl 1234.35072

[Herr et al. 2010] S. Herr, A. D. Ionescu, C. E. Kenig, and H. Koch, "A para-differential renormalization technique for nonlinear dispersive equations", Comm. Partial Differential Equations 35:10 (2010), 1827-1875. MR 2012c:35382 Zbl 1214.35089

[Ionescu and Kenig 2007] A. D. Ionescu and C. E. Kenig, "Global well-posedness of the Benjamin-Ono equation in lowregularity spaces”, J. Amer. Math. Soc. 20:3 (2007), 753-798. MR 2008f:35350 Zbl 1123.35055

[Ionescu et al. 2008] A. D. Ionescu, C. E. Kenig, and D. Tataru, "Global well-posedness of the KP-I initial-value problem in the energy space", Invent. Math. 173:2 (2008), 265-304. MR 2009h:35368 Zbl 1188.35163

[Kato 1983] T. Kato, "On the Cauchy problem for the (generalized) Korteweg-de Vries equation", pp. 93-128 in Studies in applied mathematics, edited by V. Guillemin, Adv. Math. Suppl. Stud. 8, Academic Press, New York, 1983. MR 86f:35160 Zbl 0549.34001

[Kenig and Koenig 2003] C. E. Kenig and K. D. Koenig, "On the local well-posedness of the Benjamin-Ono and modified Benjamin-Ono equations”, Math. Res. Lett. 10:5-6 (2003), 879-895. MR 2004j:35249 Zbl 1044.35072

[Kenig et al. 1991] C. E. Kenig, G. Ponce, and L. Vega, "Well-posedness of the initial value problem for the Korteweg-de Vries equation", J. Amer. Math. Soc. 4:2 (1991), 323-347. MR 92c:35106 Zbl 0737.35102

[Kenig et al. 1993] C. E. Kenig, G. Ponce, and L. Vega, "Well-posedness and scattering results for the generalized Korteweg-de Vries equation via the contraction principle", Comm. Pure Appl. Math. 46:4 (1993), 527-620. MR 94h:35229 Zbl 0808.35128 
[Koch and Tataru 2007] H. Koch and D. Tataru, "A priori bounds for the 1D cubic NLS in negative Sobolev spaces", Int. Math. Res. Not. 2007:16 (2007), Art. ID rnm053. MR 2010d:35307

[Koch and Tzvetkov 2003] H. Koch and N. Tzvetkov, "On the local well-posedness of the Benjamin-Ono equation in $H^{s}(\mathbb{R})$ ", Int. Math. Res. Not. 2003:26 (2003), 1449-1464. MR 2004b:35284 Zbl 1039.35106

[Masmoudi and Nakanishi 2005] N. Masmoudi and K. Nakanishi, "From the Klein-Gordon-Zakharov system to the nonlinear Schrödinger equation”, J. Hyperbolic Differ. Equ. 2:4 (2005), 975-1008. MR 2007e:35262 Zbl 1089.35070

[Molinet 2013] L. Molinet, "A note on the inviscid limit of the Benjamin-Ono-Burgers equation in the energy space", Proc. Amer. Math. Soc. 141:8 (2013), 2793-2798. MR 3056569 Zbl 1170.35084

[Molinet and Pilod 2012] L. Molinet and D. Pilod, "The Cauchy problem for the Benjamin-Ono equation in $L^{2}$ revisited", Anal. PDE 5:2 (2012), 365-395. MR 2970711 Zbl 1273.35096

[Molinet and Ribaud 2002] L. Molinet and F. Ribaud, "On the low regularity of the Korteweg-de Vries-Burgers equation", Int. Math. Res. Not. 2002:37 (2002), 1979-2005. MR 2003e:35272 Zbl 1031.35126

[Molinet et al. 2001] L. Molinet, J. C. Saut, and N. Tzvetkov, "Ill-posedness issues for the Benjamin-Ono and related equations", SIAM J. Math. Anal. 33:4 (2001), 982-988. MR 2002k:35281 Zbl 0999.35085

[Ott and Sudan 1970] E. Ott and R. N. Sudan, "Damping of solitary waves", Phys. Fluids 13:6 (1970), 1432-1434.

[Ponce 1991] G. Ponce, "On the global well-posedness of the Benjamin-Ono equation", Differential Integral Equations 4:3 (1991), 527-542. MR 92e:35137 Zbl 0732.35038

[Smith 1972] R. Smith, "Nonlinear Kelvin and continental-shelf waves”, J. Fluid Mech. 52 (1972), 379-391. Zbl 0235.76007

[Tao 2004] T. Tao, "Global well-posedness of the Benjamin-Ono equation in $H^{1}$ (R)", J. Hyperbolic Differ. Equ. 1:1 (2004), 27-49. MR 2005f:35273 Zbl 1055.35104

[Zhou 1997] Y. Zhou, "Uniqueness of weak solution of the KdV equation”, Int. Math. Res. Not. 1997:6 (1997), 271-283. MR 98b:35171 Zbl 0883.35105

Received 13 Jan 2015. Revised 24 Apr 2015. Accepted 21 May 2015.

LUC Molinet: luc.molinet@univ-tours.fr

Laboratoire de Mathématiques et Physique Théorique, CNRS (UMR 7350), Université François Rabelais, Fédération Denis Poisson, Faculté des Sciences et Techniques, Parc de Grandmont, 37200 Tours, France

STÉPHANE VENTO: vento@math.univ-paris13.fr

Laboratoire Analyse, Géométrie et Applications, CNRS (UMR 7539), Université Paris 13, Institut Galilée, 99 avenue J. B. Clément, 93430 Villetaneuse, France 


\title{
Analysis \& PDE
}

\author{
msp.org/apde
}

\section{EDITORS}

EDITOR-IN-CHIEF

\author{
Maciej Zworski \\ zworski@math.berkeley.edu \\ University of California \\ Berkeley, USA
}

BOARD OF EDITORS

Nicolas Burq Université Paris-Sud 11, France

nicolas.burq@math.u-psud.fr

Sun-Yung Alice Chang Princeton University, USA

chang@math.princeton.edu

Michael Christ University of California, Berkeley, USA

mchrist@math.berkeley.edu

Charles Fefferman Princeton University, USA

cf@math.princeton.edu

Ursula Hamenstaedt Universität Bonn, Germany

ursula@math.uni-bonn.de

Vaughan Jones U.C. Berkeley \& Vanderbilt University vaughan.f.jones@vanderbilt.edu

Herbert Koch Universität Bonn, Germany koch@math.uni-bonn.de

Izabella Laba University of British Columbia, Canada ilaba@math.ubc.ca

Gilles Lebeau Université de Nice Sophia Antipolis, France lebeau@unice.fr

László Lempert Purdue University, USA lempert@math.purdue.edu

Richard B. Melrose Massachussets Institute of Technology, USA rbm@math.mit.edu

Frank Merle Université de Cergy-Pontoise, France Frank.Merle@u-cergy.fr

William Minicozzi II Johns Hopkins University, USA minicozz@math.jhu.edu

Werner Müller Universität Bonn, Germany mueller@math.uni-bonn.de
Yuval Peres

Gilles Pisier

Tristan Rivière

Igor Rodnianski

Wilhelm Schlag

Sylvia Serfaty

Yum-Tong Siu

Terence Tao

Michael E. Taylor

Gunther Uhlmann

András Vasy

Dan Virgil Voiculescu

Steven Zelditch
University of California, Berkeley, USA

peres@stat.berkeley.edu

Texas A\&M University, and Paris 6

pisier@math.tamu.edu

ETH, Switzerland

riviere@math.ethz.ch

Princeton University, USA

irod@math.princeton.edu

University of Chicago, USA

schlag@math.uchicago.edu

New York University, USA

serfaty@cims.nyu.edu

Harvard University, USA siu@math.harvard.edu

University of California, Los Angeles, USA tao@math.ucla.edu

Univ. of North Carolina, Chapel Hill, USA met@math.unc.edu

University of Washington, USA gunther@math.washington.edu

Stanford University, USA andras@math.stanford.edu

University of California, Berkeley, USA

dvv@math.berkeley.edu

Northwestern University, USA

zelditch@math.northwestern.edu

PRODUCTION

production@msp.org

Silvio Levy, Scientific Editor

See inside back cover or msp.org/apde for submission instructions.

The subscription price for 2015 is US \$205/year for the electronic version, and \$390/year ( $\$ 55$, if shipping outside the US) for print and electronic. Subscriptions, requests for back issues from the last three years and changes of subscribers address should be sent to MSP.

Analysis \& PDE (ISSN 1948-206X electronic, 2157-5045 printed) at Mathematical Sciences Publishers, 798 Evans Hall \#3840, c/o University of California, Berkeley, CA 94720-3840, is published continuously online. Periodical rate postage paid at Berkeley, CA 94704, and additional mailing offices.

APDE peer review and production are managed by EditFLOw ${ }^{\circledR}$ from MSP.

\section{PUBLISHED BY}

mathematical sciences publishers

nonprofit scientific publishing

http://msp.org/

(C) 2015 Mathematical Sciences Publishers 


\section{ANALYSIS \& PDE \\ Volume $8 \quad$ No. $6 \quad 2015$}

On small energy stabilization in the NLS with a trapping potential

SCIPIO CUCCAGNA and MASAYA MAEDA

Transition waves for Fisher-KPP equations with general time-heterogeneous and spaceperiodic coefficients

\section{GRÉGOIRE NADIN and LUCA ROSSI}

Characterisation of the energy of Gaussian beams on Lorentzian manifolds: with applications 1379 to black hole spacetimes

JAN SBIERSKI

Height estimate and slicing formulas in the Heisenberg group

ROBERTO MONTI and DAVIDE VitTONE

Improvement of the energy method for strongly nonresonant dispersive equations and applications

LuC Molinet and StÉPhane Vento

Algebraic error estimates for the stochastic homogenization of uniformly parabolic equations 1497

JESSICA LIN and CHARLES K. SMART 